\title{
ESTIMAÇÃO E PREVISÃO BAYESIANAS EM DADOS AGRUPADOS
}

\section{Silvia Lopes de Paula Ferrari}

\author{
DISSERTAÇÃO APRESENTADA \\ $\mathrm{AO}$ \\ INSTITUTO DE MATEMÁTICA E ESTATÍTTICA \\ $\mathrm{DA}$ \\ UNIVERSIDADE DE SÃO PAULO \\ PARA OBTENÇÃO DO GRAU DE MESTRE \\ EM \\ ESTATISTICA \\ ÅREA DE CONCENTRAÇÃO: EJTATISTICA \\ ORIENTADOR: PROF. DR. HELENO BOLFARINE
}

- São Paulo, agosto de 1987 - 
Aos meus pais

Lucy e Edinésio

Ao meu marido

Alessandro 
AGRADECIMENTOS

Ao Prof. Dr. Heleno Bolfarine, pela sugestão do tema e pela dedicação com que me orientou.

Ao Prof. Dr. José Galvão Leite, pela colaboração na fase final do trabalho.

A Rinaldo Artes, pela enorme contribuição na parte computacional.

A Lücia Pereira Barroso, Mônica Carneiro Sandoval e Denise Aparecida Botter, pela amizade.

Aos demais professores do IME-USP, pela minha for mação.

A Antonia Soares, pelo excelente trabalho de dati lografia.

A Silvia Regina S.D.T. Oliveira e Maria Cristina Martinez Banesio, pela elaboração das referências bibliográ ficas.

Finalmente, a todos aqueles que colaboraram para a realização deste trabalho. 


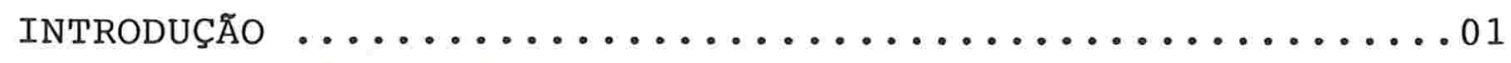

CAPITULO 1 - ESTIMAÇÃO BAYESIANA NO MODELO GERAL .......04

1.1. Introdução ................... 4

1.2. Estimadores de $\alpha$ baseados em sua distribuição, a posteriori..........06

1.2.1. Variância da distribuição a priori conhecida ..........07

1.2.2. Variância da distribuição a priori desconhecida .......15

1.3. Cálculo dos momentos a posteriori aproximados pelo método de Laplace....19

1.3.1. Variância da distribuição a priori conhecida ..........21

1.3.2. Variância da distribuição a priori desconhecida .......23

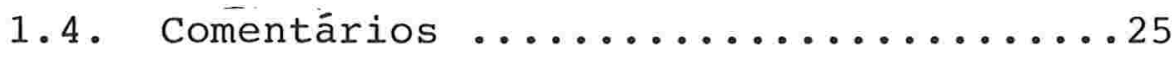

1.5. Casos particulares e extensões .....26

CAPItTULO 2 - ALGUNS MODELOS ESPECIAIS ................. 8

2.1. o modelo binomial .............28

2.1.1. Variância da distribuição a priori conhecida ..........29

2.1.2. Variância da distribuição a priori desconhecida ........31

2.1.3. Cálculo dos momentos a posteriori aproximados pelo método de Laplace ................32 
2.2. O modelo de Poisson ..............32

2.2.1. Variância da distribuição

a priori conhecida .........33

2.2.2. Variância da distribuição

a priori desconhecida .......35

2.2.3. Cálculo dos momentos a posteriori aproximados pelo método de Laplace .........36

2.3. o modelo normal ................36

2.3.1. Variâncias conhecidas ......37

2.3.2. Variâncias desconhecidas ....38

2.3.3. Cálculo dos momentos a posteriori aproximados pelo método de Laplace .........42

\section{CAPITULO 3 - PREVISÃO BAYESIANA EM AMOSTRAGEM EM DOIS} ESTÁGIOS $\ldots \ldots \ldots \ldots \ldots \ldots \ldots \ldots \ldots \ldots$

3.1. Introdução $\ldots \ldots \ldots \ldots \ldots \ldots \ldots 4$

3.1.1. O enfoque de superpopulação para populações finitas .....44

3.1.2. Amostragem em dois estágios...45

3.2. o modelo geral .................45

3.2.1. Variância da distribuição a priori conhecida .........49

3.2.2. Variância da distribuição a priori desconhecida .......52

3.3. Cálculo dos momentos a posteriori aproximados pelo método de Laplace....54

3.3.1. Variầncia da distribuição $a$ priori conhecida .........54

3.3.2. Variância da distribuição a priori desconhecida .......56 
3.4. Alguns modelos especiais ..........58

3.4.1. o modelo binomial ..........58

3.4.2. o modelo de Poisson .........59

3.4.3. o modelo normal ............59

3.4.3.1. Variâncias conhecidas 60

3.4.3.2. Variâncias

desconhecidas .....66

CAPITULO 4 - APLICAÇÕES EM AMOSTRAS SIMULADAS ........68

4.1. Introdução .................68

4.2. Estimação no modelo binomial .......69

4.3. Estimação no modelo de Poisson ......75

4.4. Estimação no modelo normal ........77

4.5. Previsão em amostragem em dois estágios ...........................

4.5.1. Previsão no modelo binomial...79

4.5.2. Previsão no modelo de Poisson.83

4.5.3. Previsão no modelo normal ...84

APENDICE 1 - Cálculo dos momentos a posteriori aproximados pelo método de Laplace .......86

APENDICE 2 - Saída e listagem do programa que calcula as estimativas modais de $\boldsymbol{\theta}$. Modelo binomial 90

APENDICE 3 - Saida e listagem do programa que calcula as estimativas de $\boldsymbol{\theta}$ basearas no método de Laplace. Modelo binomial ............97 
APÊNDICE 4 - Saída e listagem do programa que calcula as estimativas modais de $\theta$. Modelo de Poisson 103

APÊNDICE 5 - Saída e listagem do programa que calcula as estimativas modais de $\theta$ baseadas no método de Laplace. Modelo de Poisson ............ 110

APÊNDICE 6 - Saída e listagem do programa que calcula as estimativas de $\boldsymbol{\theta}$. Modelo normal ....... 116

APÊNDICE 7 - Amostragem em dois estágios - Populações e amostras simuladas. Modelo binomial ..... 123

APÊNDICE 8 - Amostragem em dois estágios - Populações e amostras simuladas. Modelo de Poisson .... 125

APÊNDICE 9 - Amostragem em dois estágios - Populações e amostras simuladas. Modelo normal ...... 127

REFERÊNCIAS BIBLIOGRÁFICAS $\ldots \ldots \ldots \ldots \ldots \ldots \ldots \ldots \ldots \ldots \ldots$

০oo 


\section{INTRODUÇÃO}

o objetivo deste trabalho é apresentar uma metodolo gia Bayesiana de estimação em dados agrupados e de previsão em amostragem em dois estágios.

A expressão "dados agrupados" é utilizada para caracterizar a seguinte situação: $n$ populações distintas são consideradas e de cada uma delas é retirada uma amostra alea tória formando, portanto, n grupos de dados. O interesse é estimar simultaneamente as médias $\theta_{1}, \ldots, \theta_{n}$ associadas a cada uma das $\mathrm{n}$ populações.

É comum, na prática, nos depararmos com situações em que é razoável supor que a distribuição dos dados é binomial, Poisson ou normal. Assim, apresentaremos uma metodolo gia em que a distribuição dos dados pertence à família exponencial de distribuições, da qual as distribuições acima são simplesmente casos particulares. Especificamente, assumiremos que $Y_{i j}$, a j-ésima observação da i-ésima amostra tem fun ção de densidade (ou de probabilidade) da forma

$$
\pi\left(y_{i j} / \alpha_{i}, \phi_{i}\right) \propto \exp \left\{\phi_{i}\left[\alpha_{i} y_{i j}-a\left(\alpha_{i}\right)\right]\right\}
$$

onde $\mathrm{a}(\cdot)$ é uma função conhecida e derivável até segunda ordem, $\phi_{i}>0$ é conhecido e chamado de parâmetro de escala e $\alpha_{i}$, denominado parâmetro canônico ou natural, é uma função do parâmetro de interesse $\Theta_{i}$. 
Assumiremos ainda a seguinte estrutura a priori em dois estágios para os parâmetros canônicos:

Estágio 1. $\alpha_{i} / \mu, \sigma^{2} \sim N\left(\mu, \sigma^{2}\right), i=1, \ldots, n$, independentes.

Estágio 2. $\pi(\mu) \propto$ constante, $\sigma^{2}$ conhecida, e para o caso em que $\sigma^{2}$ é desconhecida,

$$
\frac{\nu \lambda}{\sigma^{2}} \mid \nu, \lambda \sim x_{\nu}^{2}, \nu>0, \lambda>0,
$$

onde $\mu$ e $\sigma^{2}$, dados $\nu$ e $\lambda$, são independentes. Esta estrutu彑 ra a priori parece ser razoável em muitas situações e dare- mos justificativas para a sua utilização.

os parâmetros de interesse, ou seja, $\theta_{1}, \ldots, \theta_{n}$, serão estimados através da distribuição a posteriori de $\alpha^{T}=\left(\alpha_{1}, \ldots, \alpha_{n}\right)$ por métodos aproximados dentre os quais um método baseado em aproximações de Laplace para integrais.

Quanto à previsão Bayesiana em amostragem em dois estágios, o enfoque utilizado será o de superpopulação, sendo que a distribuição dos dados será também da família exponencial e a estrutura a priori, a mesma citada anteriormente. Proporemos previsores para combinações lineares dos elemen tos da população como, por exemplo, o total e a média popula cionais.

No Capítulo 1 trataremos do problema de estimação no modelo geral, isto é, aquele em que a distribuição dos dạ dos pertence à família exponencial de distribuições. Serão propostas aproximações para a distribuição a posteriori de $\alpha$ e para os estimadores dos parâmetros de interesse $\theta_{1}, \ldots, \theta_{n}$. 
No Capítulo 2 todos os resultados encontrados no Ca pítulo 1 serão aplicados às distribuições binomial, Poisson e normal. Nesta última será feita uma extensão ao caso em que as variâncias populacionais são desconhecidas.

No Capítulo 3 apresentaremos uma metodologia de previsão Bayesiana em amostragem em dois estágios tanto para o modelo geral quanto para os casos particulares em que a distribuição dos dados é binomial, poisson ou normal. Para a distribuição normal será feita uma extensão para a situa ção em que as variâncias populacionais são desconhecidas.

Finalmente, no Capitulo 4 apresentaremos resultados numéricos baseados em amostras simuladas, bem como faremos comparações entre as diversas aproximações propostas. 
CAPITULO 1

ESTIMAÇÃO BAYESIANA NO MODELO GERAL

\subsection{Introdução}

o objetivo deste capítulo é apresentar uma metodolo gia de estimação Bayesiana em dados agrupados. A situação que se apresenta é a seguinte: n populações são consideradas; de cada uma é retirada uma amostra; denotamos as n amostras a través dos vetores $\mathbf{x}_{1}, \ldots, \mathbf{Y}_{n}$ e seus tamanhos, respectivamente, por $m_{1}, \ldots, m_{n}$; assumimos que, dado $\theta_{i}, Y_{i j}$, a j-ésima observa ção da i-ésima amostra, tem média $\Theta_{i}$ e nosso objetivo é estimar, simultaneamente, $\theta_{1}, \ldots, \theta_{n}$; assumimos também que, dados $\alpha_{i}$ e $\phi_{i}, x_{i j}$ tem função de densidade (ou de probabilidade) da fá mîlia exponencial de distribuições, isto é,

$$
\pi\left(y_{i j} \mid \alpha_{i}, \phi_{i}\right)=\exp \left\{\phi_{i}\left[y_{i j} \alpha_{i}-a\left(\alpha_{i}\right)\right]\right\} b\left(y_{i j}, \phi_{i}\right)
$$

onde $\mathrm{a}(\cdot)$ e $\mathrm{b}(\cdot, \cdot)$ são funções conhecidas, $\mathrm{a}(\cdot)$ é derivável $\underline{\mathrm{a}}$ té segunda ordem, $\phi_{i}>0$ é conhecido e chamado parâmetro de escala e $\alpha_{i}$ é chamado parâmetro canônico ou natural.

Dois resultados bastante úteis e válidos para o modelo acima são

$$
\begin{aligned}
& E\left[Y_{i j} / \alpha_{i}\right]=a^{\prime}\left(\alpha_{i}\right)=\theta_{i} \text { e } \\
& \operatorname{Var}\left[Y_{i j} / \alpha_{i}\right]=\frac{a^{\prime \prime}\left(\alpha_{i}\right)}{\phi_{i}}, i=1, \ldots, n .
\end{aligned}
$$

(*) $a^{\prime}(\cdot)$ e a" $(\cdot)$ denotam, respectivamente, as derivadas de primeira e segunda ordem da função $a(\cdot)$. 
Este modelo é um particular Modelo Linear Generalizado definido, por exemplo, em McCullagh e Nelder (1983) e Cordeiro (1986). Alguns resultados sobre inferência Bayesiana nos Modelos Lineares Generalizados são encontrados em West (1985) .

Três importantes distribuições, dentre outras, são casos particulares do modelo em (1.1) (que chamaremos de MODELO GERAL) :

i) Distribuição binomial

Tomando $\phi_{i}=1, \alpha_{i}=\log \left[\Theta_{i} /\left(1-\Theta_{i}\right)\right], 0<\theta_{i}<1$, $a\left(\alpha_{i}\right)=\log \left(1+e^{\alpha^{i}}\right)$ e $y_{i j}$ igual a zero ou um, temos que $\mathrm{Y}_{i j}$, dado $\Theta_{i}$, tem distribuição de Bernoulli com probabilida de sucesso $\theta_{i}$ ou, equivalentemente, que $Y_{i}=\sum_{j=1}^{m_{i}} Y_{i j}$, dados $\Theta_{i}$ e $m_{i}$, tem distribuição binomial com probabilidades de sucesso $\theta_{i}$ e indice $m_{i}$, que denotamos por

$$
\mathrm{Y}_{i} / \mathrm{m}_{i}, \Theta_{i} \sim \mathrm{B}\left(\mathrm{m}_{i}, \Theta_{i}\right), i=1, \ldots, \mathrm{n} .
$$

ii) Distribuição de Poisson

Para obter a distribuição de Poisson com média $\theta_{i}$, basta tomar $\phi_{i}=1, \alpha_{i}=\log _{i}, a\left(\alpha_{i}\right)=e^{\alpha_{i}}$ e $y_{i j}$ inteiro não negativo. Neste caso, utilizamos a seguinte notação:

$$
Y_{i j} / \Theta_{i} \sim P\left(\Theta_{i}\right), i=1, \ldots, n, j=1, \ldots, m_{i} .
$$

\section{iii) Distribuição normal}

A distribuição normal com média $\Theta_{i}$ e variância $\sigma_{i}^{2}$ é obtida tomando-se $\phi_{i}=1 / \sigma_{i}^{2}, \alpha_{i}=\theta_{i}$ e a $\left(\alpha_{i}\right)=\sigma_{i}^{2} / 2$. 
Neste caso, a notação utilizada é:

$$
\mathrm{Y}_{i j} / \Theta_{i}, \sigma_{i}^{2} \sim N\left(\Theta_{i}, \sigma_{i}^{2}\right)
$$

Na realidade, o que desenvolvemos neste capítulo é uma generalização do estudo feito por Leonard (1972) para da dos binomiais agrupados, isto é, estudamos o modelo geral do qual o modelo binomial é um caso particular.

\subsection{Estimadores de $\alpha$ baseados em sua distribuição a posteriori}

Consideremos $\mathrm{n}$ amostras aleatórias independentes $\mathbf{y}_{1}, \ldots, \mathbf{Y}_{\mathrm{n}}$ com $\mathrm{m}_{1}, \ldots, \mathrm{m}_{\mathrm{n}}$ observações respectivamente tais que a função de densidade (ou de probabilidade) de $\mathrm{Y}_{i j}$, a j-é sima observação da i-ésima amostra, seja da forma

$$
\begin{aligned}
& \pi\left(y_{i j} / \alpha_{i}, \phi_{i}\right) \propto \exp \left\{\phi_{i}\left[y_{i j} \alpha_{i}-a\left(\alpha_{i}\right)\right]\right\} \\
& i=1, \ldots, n, j=1, \ldots, m_{i},
\end{aligned}
$$

onde $\alpha_{i}, a(\cdot)$ e $\phi_{i}$ estão definidos em (1.1).

A função de verossimilhança de $\alpha$ é, portanto,

$$
\begin{aligned}
L\left(\alpha / \mathbf{y}_{1}, \ldots, \mathbf{y}_{\mathrm{n}}, \phi\right) \propto \prod_{i=1}^{n} \prod_{j=1}^{m_{i}} \exp \left\{\phi_{i}\left[y_{i j} \alpha_{i}-a\left(\alpha_{i}\right)\right]=\right. \\
=\exp \left\{\sum_{i=1}^{n} \phi_{i}\left[y_{i} \alpha_{i}-m_{i} a\left(\alpha_{i}\right)\right]\right\}
\end{aligned}
$$

onde $y_{i}=\sum_{j=1}^{m_{i}} y_{i j}, i=1, \ldots, n, \alpha^{T}=\left(\alpha_{1}, \ldots, \alpha_{n}\right)$ e $\phi^{T}=\left(\phi_{1}, \ldots, \phi_{n}\right)$. 
1.2.1. Variância da distribuição a priori conhecida Tomemos a seguinte estrutura a priori em dois estágios:

Estágio 1. $\alpha_{i} / \mu, \sigma^{2} \sim N\left(\mu, \sigma^{2}\right), i=1, \ldots, n$, independentes;

Estágio 2. $\pi(\mu) \propto$ constante.

Estudamos, em primeiro lugar, o caso em que $\sigma^{2}$ é conhecida. A seguir, proporemos também uma distribuição a pri ori para $\sigma^{2}$.

A distribuição a priori de $\alpha$ acima é permutável no sentido dado por De Finetti (1964), isto é, invariante sob a permutação dos índices. A suposição de permutabilidade é vá lida quando se tem a mesma informação a priori sobre cada $\alpha_{i}$ e, da mesma forma, sobre cada $\operatorname{par}\left(\alpha_{i}, \alpha_{j}\right)$, $i \neq j$, e o mesmo para cada tripla $\left(\alpha_{i}, \alpha_{j}, \alpha_{k}\right), i \neq j \neq k$, e assim sucessivamente. Deste modo, a metodologia que apresentaremos só de ve ser aplicada se esta suposição é válida, caso contrário, uma outra distribuição a priori mais conveniente deve ser con siderada. Na verdade, estamos utilizando uma forma particular de distribuição permutável, isto é, aquela em que $\alpha_{1} \ldots, \alpha_{n}$ formam uma amostra aleatória de uma distribuição normal cuja variância $\sigma^{2}$ é conhecida e cuja méd:a $\mu$ tem ainda uma distrí buição imprópria proporcional a uma constante. Embora esta seja imprópria, a distribuição a posteriori será própria e, portanto, não há porque se preocupar. 
Na realidade, as distribuições a priori considera das foram escolhidas pelas facilidades técnicas que elas prọ porcionam. No entanto, não vemos nenhum inconveniente em utilizá-las. Tomemos por exemplo o caso da distribuição bino mial em que é usual se tomar uma distribuição a priori beta para os parâmetros $\theta_{1}, \ldots, \theta_{n}$. Agora, se lembrarmos que se $\alpha_{i}$ tem distribuição normal, então $\Theta_{i}$ tem aproximadamente distri buição beta (Leonard, 1972), vemos que é perfeitamente plausivel assumir que $\alpha_{1}, \ldots, \alpha_{n}$ sejam normalmente distribuidos. Uma breve discussão sobre o uso de prioris normais é encon trada em West (1985, Seção 4.1).

Ainda cabe observar que os estimadores que proporemos para um particular $\alpha_{i}$ serão baseados nas observações de to das as n amostras e não sónas da i-ésima. Isto é realmente de se esperar uma vez que assumimos a priori que $\alpha_{i}, \ldots, \alpha_{n}$ formam uma amostra aleatória de uma mesma distribuição e,por tanto, as observações que fornecem informação sobre $\alpha_{i}$ devem também fornecer sobre $\alpha_{j}, i \neq j$.

Assim, dadas a função de verossimilhança em (1.3) e a estrutura a priori em (1.4) e (1.5) é fácil encontrar a função de densidade a posteriori conjunta de $\alpha$ e $\mu$, isto é,

$$
\begin{aligned}
\pi\left(\alpha, \mu / \mathbf{y}_{1}, \ldots, \mathbf{y}_{\mathrm{n}}, \phi, \sigma^{2}\right) & \propto L\left(\alpha / \mathbf{y}_{1}, \ldots, \mathbf{y}_{\mathrm{n}}, \phi\right) \times \\
& \times \pi\left(\alpha / \mu, \sigma^{2}\right) . \pi(\mu)
\end{aligned}
$$

$$
\propto \exp \left\{\sum_{i=1}^{n} \phi_{i}\left[y_{i} \alpha_{i}-m_{i} a\left(\alpha_{i}\right)\right]-\frac{1}{2 \sigma^{2}} \sum_{i=1}^{n}\left(\alpha_{i}-\mu\right)^{2}\right\}
$$


A integração de (1.6) em relação a $\mu$ produz a fun ção de densidade a posteriori marginal de $\alpha$ abaixo:

$$
\begin{aligned}
\pi\left(\alpha / \mathbf{y}_{1}, \ldots, \mathbf{y}_{n}, \phi, \sigma^{2}\right) \propto \exp \left\{\sum_{i=1}^{n} \phi_{i}\left[y_{i} \alpha_{i}-m_{i} a\left(\alpha_{i}\right)\right]-\right. \\
\left.-\frac{1}{2 \sigma^{2}} \sum_{i=1}^{n}\left(\alpha_{i}-\alpha .\right)^{2}\right\}
\end{aligned}
$$

onde $\alpha_{\bullet}=\sum_{i=1}^{n} \frac{\alpha_{i}}{n}$. É fácil ver que (1.7) é o produto da função de verossimilhança por uma função de densidade normal multivariada.

o estimador de Bayes de $\boldsymbol{\alpha}$ (com relação à perda quadrática) é a média da distribuição a posteriori acima. o cál culo desta média é bastante complicado pelo fato de envolver integrações que nem sempre podem ser resolvidas analiticamen te. Para evitar esta dificuldade estimamos $\alpha$ através da moda da distribuição a posteriori. Para encontrar tal estima dor basta maximizar (1.7) em relação a $\alpha$. Assim, derivando o logaritmo da função de densidade a posteriori e igualan do a zero, temos que os estimadores modais são dados pela so lução do sistema de equações:

$$
a^{\prime}\left(\alpha_{i}\right)=\frac{y_{i}}{m_{i}}-\frac{\tilde{\alpha}_{i}-\tilde{\alpha}_{i}}{m_{i} \phi^{2}}, \quad i=1, \ldots, n,
$$

onde $\tilde{\alpha}_{\bullet}=\sum_{i=1}^{n} \frac{\tilde{\alpha}_{i}}{n}$

Como foi observado na seção $1.1, \quad a^{\prime}\left(\alpha_{i}\right)$ é a média $\theta_{i}$ de $Y_{i j}$ e, portanto, $a^{\prime}\left(\tilde{\alpha}_{i}\right)=\tilde{\theta}_{i}$ é um estimador natural de $\Theta_{i}$, o parâmetro de interesse. Quando $\sigma^{2}$ ou $m_{i}$ (ou ambos) é razoavelmente grande, $\tilde{\Theta}_{i}$ nada mais é do que a média das 
observações do i-ésimo grupo. O mesmo ocorre quando $\phi_{i}$ é grande (deve-se lembrar que $\phi_{i}$ é inversamente proporcional a $\left.\operatorname{Var}\left[\mathrm{Y}_{i j} / \alpha_{i}\right]\right)$. Nestes casos a influência da estrutura a prio ri é praticamente nula.

Dependendo da função $a(\cdot)$, a solução de (1.8) só pô de ser encontrada por um processo iterativo. o método de Newton Raphson em n dimensões (Box, Davies e Swan, 1969, pg. 36) pode ser usado. Este método implica na solução iterativa do sistema de equações

$$
\alpha_{(k+1)}=\alpha_{(k)}+c_{(k)}^{-1} c_{(k)}
$$

onde $\alpha_{(\mathrm{k})}$ denota o vetor $\alpha$ resultante da k-ésima iteração do processo, $\mathbf{c}_{(\mathrm{k})}$ denota a matriz cujo elemento $(i, j)$ é

$$
c_{i j(k)}=-\left.\frac{\partial^{2} \log \pi\left(\alpha / \mathbf{y}_{1}, \ldots, \mathbf{y}_{n}, \phi, \sigma^{2}\right)}{\partial \alpha_{i} \partial \alpha_{j}}\right|_{\alpha=\alpha_{(k)}}, i, j=1, \ldots, n
$$

e $\mathbf{c}_{(\mathrm{k})}$ denota o vetor cujo elemento i é

$$
c_{i(k)}=\left.\frac{\partial \log \pi\left(\alpha / \mathbf{Y}_{1}, \ldots, \mathbf{y}_{n}, \phi, \sigma^{2}\right)}{\partial \alpha_{i}}\right|_{\alpha=\alpha_{(k)}} \quad, i=1, \ldots, n .
$$

Estabelecendo o vetor inicial $\alpha_{(0)}, \alpha_{(1)}$ pode ser calculado através de (1.9); com este novo vetor, $\alpha_{\text {(2) po }}$ de ser agora calculado e assim, sucessivamente, até a obtenção de convergência (o que não é garantido), isto é, até que

$$
\left|\frac{\alpha_{i(k+1)}-\alpha_{i(k)}}{\alpha_{i(k)}}\right|, \quad i=1, \ldots, n
$$

seja suficientemente pequeno. 
Assim, para encontrar a solução de (1.8) basta utilizar o método de Newton Raphson com

$$
\begin{aligned}
& c_{i(k)}=\phi_{i} y_{i}-m_{i} \phi_{i} a^{\prime}\left(\alpha_{i(k)}\right)-\frac{\alpha_{i(k)}-\alpha \cdot(k)}{\sigma^{2}}, \\
& i=1, \ldots, n,
\end{aligned}
$$

e com o elemento $(i, j)$ da matriz $\mathbf{C}_{(k)}$ dado por

$$
C_{i j(k)}=\frac{\delta_{i j}}{u_{i(k)}}-\frac{1}{n \sigma^{2}}, i, j=1, \ldots, n
$$

onde $u_{i(k)}=\left(m_{i} \phi_{i} a^{\prime \prime}\left(\alpha_{i(k)}\right)+\frac{1}{\sigma^{2}}\right)^{-1}, \quad i=1, \ldots, n$

e

$$
\delta_{i j}=\left\{\begin{array}{ll}
1, & \text { se } i=j \\
0, & \text { se } i \neq j
\end{array} \quad, i, j=1, \ldots, n\right. \text {. }
$$

A inversa da matriz $\mathbf{C}_{(\mathrm{k})}$, que denotamos por $\mathbf{D}_{(\mathrm{k})}$, tem os seguintes elementos:

$$
D_{i j(k)}=u_{i(k)} \delta_{i j}+\frac{u_{i(k)} u_{j}(k)}{n\left(\sigma^{2}-u_{\bullet(k)}\right)}, i, j=1, \ldots, n .(1.11)
$$

onde $u_{\cdot(k)}=\sum_{i=1}^{n} \frac{u_{i(k)}}{n}$. Logo, não é necessário inverter numericamente a matriz $\mathbf{c}_{(\mathrm{k})}$ mas sim substituir $\mathbf{c}_{(\mathrm{k})}^{-1}$ por $\mathbf{D}_{(\mathrm{k})} \cdot$

E possivel, em grandes amostras, aproximar a função de densidade a posteriori por uma normal multivariada com mé dia igual à moda a posteriori e matriz de covariância dada pe lo inverso da matriz de informação observada a posteriori calculada no ponto modal. Esta aproximação é justificada ex (*) - A função indicadora $\delta$ serà utilizada em todo o trabalho. 
pandindo-se a função de densidade a posteriori em torno da moda a posteriori através de uma série de Taylor e truncando após o termo quadrático da expansão (Zellner, 1971). A média e a matriz de covariância desta distribuição aproximada são obtidas, respectivamente, através do vetor $\alpha_{(k)}$ e da matriz $D_{(k)}$ da última iteração do processo de Newton Raphson. Assim, podem ser feitas inferências aproximadas sobre o ve tor de parâmetros a como, por exemplo, construir intervalos de credibilidade para cada $\alpha_{i}$ ou para diferenças do tipo $\alpha_{i}-\alpha_{j}(i \neq j)$.

Para evitar a utilização de métodos iterativos e ví sualizar melhor a forma do estimador de $\alpha$, seria interessan te encontrarmos uma solução explícita aproximada para o sistema de equações em (1.8). Esta pode ser obtida se fizermos uma aproximação normal para a função de verossimilhança de $\alpha$.

A função de verossimilhança de $\alpha$ pode ser aproximada, em grandes amostras, por uma densidade normal-multivariada com vetor de médias igual à estimativa de máxima verossimilhança $\hat{\alpha}^{\mathrm{T}}=\left(\hat{\alpha}_{1}, \ldots, \hat{\alpha}_{n}\right)$ e matriz de covariância dada pelo inverso da matriz cujo elemento $(i, j)$ é

$$
-\left.\frac{\partial^{2} \log L(\alpha / y)}{\partial \alpha_{i} \partial \alpha_{j}}\right|_{\alpha=\hat{\alpha}} \quad, i, j=1, \ldots, n \text {. }
$$

Esta aproximação é justificada expandindo-se a função de verossimilhança numa série de Taylor em torno de $\hat{\alpha}$ e truncando após o termo quadrático da expansão (Zellner, 1971) .

É fácil ver que

$$
\hat{\alpha}_{i}=a^{\prime-1}\left(\frac{y_{i}}{m_{i}}\right), \quad i=1, \ldots, n \text {, }
$$


$\left(a^{-1}(\cdot)\right.$ denota a função inversa de $\left.a^{\prime}(\cdot)\right)$ e que a matriz de covariância tem elementos da diagonal principal dados por

$$
v_{i}=\left[m_{i} \phi_{i} a^{n}\left(\hat{\alpha}_{i}\right)\right]^{-1} \quad, \quad i=1, \ldots, n
$$

e os demais iguais a zero.

Assim, com esta função de verossimilhança aproximada e assumindo a estrutura a priori dada por (1.4) e (1.5), temos que a função de densidade a posteriori é $\pi *\left(\alpha / \mathbf{y}_{1}, \ldots, \mathbf{y}_{\mathrm{n}}, \phi, \sigma^{2}\right) \propto \exp \left\{-\frac{1}{2} \sum_{i=\uparrow}^{n} \frac{\left(\alpha_{i}-\hat{\alpha}_{i}\right)^{2}}{v_{i}}-\frac{1}{2 \sigma^{2}} \sum_{i=1}^{n}\left(\alpha_{i}-\alpha_{0}\right)^{2}\right\}$. Esta é uma densidade normal multivariada uma vez que o termo exponencial é uma função quadrática de $\alpha_{i}$. Encontrar a média a posteriori é bastante simples uma vez que, na distribui ção normal, a média é igual à moda. Então, derivando o logaritmo da função de densidade $\pi$ * acima e igualando a zero temos:

$$
-\frac{\left(\tilde{\alpha}_{i}^{*}-\hat{\alpha}_{i}\right)}{v_{i}}-\frac{\left(\tilde{\alpha}_{i}^{*}-\tilde{\alpha}_{\bullet}^{*}\right)}{\sigma^{2}}=0, \quad i=1, \ldots, \mathrm{n} .
$$

Após alguns cálculos obtemos

$$
\tilde{\alpha}_{i}^{*}=\frac{\frac{\hat{\alpha}_{i}}{v_{i}}+\frac{\hat{\alpha}}{\sigma^{2}}}{\frac{1}{v_{i}}+\frac{1}{\sigma^{2}}}, i=1, \ldots, n,
$$

onde

$$
\hat{\alpha}_{0}=\frac{\sum_{i=1}^{n} \frac{\hat{\alpha}_{i}}{\sigma^{2}+v_{i}}}{\sum_{i=1}^{n} \frac{1}{\sigma^{2}+v_{i}}} \text {. }
$$


Logo $\tilde{\alpha}_{i}^{*}$ é uma média ponderada entre $\hat{\alpha}_{i}$, o estimador de máxima verossimilhança de $\alpha_{i}$ e $\hat{\alpha}_{\bullet}$, com pesos iguais aos inversos de $v_{i}$ e $\sigma^{2}$ respectivamente. (1.12) pode ser rees crita como

$$
\tilde{\alpha}_{i}^{*}=d_{i} \hat{\alpha}_{i}+\left(1-d_{i}\right) \hat{\alpha}_{\cdot},
$$

onde $d_{i}=\sigma^{2} /\left(\sigma^{2}+v_{i}\right)$ e $\hat{\alpha} \cdot=\left(\sum_{i=1}^{n} d_{i} \hat{\alpha}_{i}\right) / \sum_{i=1}^{n} d_{i} \cdot$ As estimativas dos $\alpha_{i}$ 's são então "contraidas" em direção a $\hat{\alpha}$. e esta contração é bem mais acentuada quando $\sigma^{2}$ é pequena quando comparada com $v_{i}$ (note-se que $0 \leqq d_{i} \leqq 1$ ).

Denotemos por $\tilde{\mathbf{C}} *$ a matriz cujos elementos são

$$
\begin{aligned}
\tilde{c}_{i j}^{*} & =-\left.\frac{\partial^{2} \log \pi *\left(\alpha / y_{1}, \ldots, y_{n}, \phi, \sigma^{2}\right)}{\partial \alpha_{i} \partial \alpha_{j}}\right|_{\alpha=\tilde{\alpha} *} \\
& =\frac{\delta_{i j}}{z_{i}}-\frac{1}{n \sigma^{2}}, \quad i, j=1, \ldots, n,
\end{aligned}
$$

onde

$$
z_{i}=v_{i} \frac{\sigma^{2}}{\sigma^{2}+v_{i}}=v_{i} d_{i}, i=1, \ldots, n
$$

A inversa da matriz $\tilde{\mathbf{C}} *$, denotada por $\tilde{D}^{*}$, é a matriz de covariância da distribuição a posteriori aproximada e tem elementos dados por:

$$
\tilde{D}_{i j}^{*}=z_{i} \delta_{i j}+\frac{z_{i} z_{j}}{n\left(\sigma^{2}-z_{.}\right)}
$$

onde

$$
z_{0}=\sum_{i=1}^{n} \frac{z_{i}}{n}
$$


Com esta aproximação podemos fazer inferências aprọ ximadas sobre o vetor de parâmetros $\alpha$, com a vantagem de que temos expressões explícitas para os estimadores de $\alpha_{i}$, $i=1, \ldots, n$. No Capítulo 5 faremos comparações numéricas en tre as duas aproximações propostas.

\subsubsection{Variância da distribuição a priori desconhecida}

Analisamos agora o caso mais comum na prática, ou seja, aquele em que $\sigma^{2}$, a variância da distribuição a priori, é desconhecida. Tomemos a estrutura a priori da seguinte forma:

Estágio 1. $\alpha_{i} / \mu, \sigma^{2} \sim N\left(\mu, \sigma^{2}\right), i=1, \ldots, n$,

independentes.

Estágio 2. $\pi(\mu) \propto$ constante e

$$
\frac{\nu \lambda}{\sigma^{2}} / \nu, \lambda \sim x_{\nu}^{2} \quad, \nu>0, \lambda>0
$$

onde $\mu$ e $\sigma^{2}$, dados $\nu$ e $\lambda$, são independentes. Aqui, $\lambda$ é o va lor que se espera, a priori, para a variância $\sigma^{2}$, enquanto que $\nu$ é uma medida de precisão, ou seja, de quanta certeza se tem a priori de que $\lambda$ é um valor razoável para $\sigma^{2}$. Quando $\nu \rightarrow 0$, tem-se a priori usual (e imfrópria) proporcional a $\sigma^{-2}$. Quando se tem pouca ou nenhuma informação sobre $\sigma^{2}$, seria natural tomar $\nu=0$, neste caso, não haveria necess dade de especificar um valor para $\lambda$. No entanto, a distri- 
buăigão a posteriori também seria imprópria, como veremos a se guir. Então, neste caso, deve-se tomar $v$ bastante pequeno e, portanto, o valor especificado para $\lambda$ praticamente não influ enciará os resultados. Uma discussão sobre as propriedades da distribuição assumida para $\sigma^{2}$, isto é, $x^{2}$-invertida, é en contrada em Lindley (1965, Seção 5.3) .

A função de densidade a priori de $\sigma^{2}$ é $\pi\left(\sigma^{2}\right) \propto\left(\sigma^{2}\right)^{-\frac{1}{2}(\nu+2)} \exp \left\{-\frac{1}{2} \frac{\nu \lambda}{\sigma^{2}}\right\}$.

A função de densidade a posteriori conjunta de $\alpha$, $\mu$ e $\sigma^{2}$ é dada por

$$
\begin{aligned}
& \pi\left(\alpha, \mu, \sigma^{2} / \mathbf{Y}_{1}, \ldots, \mathbf{Y}_{\mathrm{n}}, \nu, \lambda, \phi\right) \propto L\left(\alpha / \mathbf{Y}_{1}, \ldots, \mathbf{Y}_{\mathrm{n}}, \phi\right) \pi\left(\alpha / \mu, \sigma^{2}\right) \pi(\mu) \pi\left(\sigma^{2} / \nu, \lambda\right) \\
& \propto\left(\sigma^{2}\right)^{-\frac{1}{2}(\nu+n+2)} \exp \left\{\sum_{i=1}^{\mathrm{n}} \phi_{i}\left[y_{i} \alpha_{i}-m_{i} a\left(\alpha_{i}\right)\right]-\frac{1}{2 \sigma^{2}}\left[\nu \lambda+\sum_{i=1}^{n}\left(\alpha_{i}-\mu\right)^{2}\right]\right\} .
\end{aligned}
$$

Integrando-se a função acima com respeito a $\mu$ temos

$\pi\left(\alpha, \sigma^{2} / \boldsymbol{y}_{1}, \cdots, \boldsymbol{y}_{\mathrm{n}}, \nu, \lambda, \phi\right) \propto\left(\sigma^{2}\right)^{-\frac{1}{2}(\nu+n+1)} \exp \left\{\sum_{i=1}^{n} \phi_{i}\left[y_{i} \alpha_{i}-m_{i} a\left(\alpha_{i}\right)\right]-\frac{1}{2 \sigma^{2}}\left[\nu \lambda+\sum_{i=1}^{n}\left(\alpha_{i}-\alpha_{0}\right)^{2}\right]\right\}$

A integração com respeito a $\sigma^{2}$ é bastante simples se usarmos o seguinte resultado:

$$
\int_{0}^{\infty}\left(\sigma^{2}\right)^{-q} \exp \left\{-\frac{Q}{\sigma^{2}}\right\} d \sigma^{2} \propto Q^{-(q-1)}
$$

Fazendo a integração com respeito a $\sigma^{2}$, temos

$$
\pi\left(\alpha / y_{1}, \cdots, \mathbf{y}_{n}, \nu, \lambda, \phi\right) \propto\left[\nu \lambda+\sum_{i=1}^{n}\left(\alpha_{i}-\alpha_{0}\right)^{2}\right]^{-\frac{1}{2}(\nu+n-1)} \exp \left\{\sum_{i=1}^{n} \phi_{i}\left[y_{i} \alpha_{i}-m_{i} a\left(\alpha_{i}\right)\right]\right\}
$$


A função de densidade acima é proporcional ao produto da função de verossimilhança por uma função de densidade $t$ multivariada. Note-se que $v$ não pode ser igual a zero, pois, neste caso, o primeiro fator do produto em (1.16) ten deria a $\infty$ quando $\alpha_{1}=\ldots=\alpha_{n}$. Isto demonstra o que foi di to acima, ou seja, que se uma densidade a priori proporcio nal a $\sigma^{2}$ fosse tomada, a densidade a posteriori seria imprópria.

Derivando o logaritmo de $\pi\left(\alpha / \mathbf{Y}_{1}, \ldots, \mathbf{y}_{n}, \nu, \lambda, \phi\right)$ com respeito $\mathrm{a} \alpha_{i}, i=1, \ldots, \mathrm{n}$ e igualando a zero, obtemos o sis tema de equações abaixo cuja solução fornece as estimativas modais:

$$
\mathrm{a}^{\prime}\left(\tilde{\alpha}_{i}\right)=\frac{y_{i}}{m_{i}}-\frac{\tilde{\alpha}_{i}-\tilde{\alpha}_{0}}{m_{i} \phi_{i} \tilde{\sigma}^{2}}, i=1, \ldots, n,
$$

onde

$$
\begin{aligned}
& \tilde{\sigma}^{2}=\frac{\nu \lambda+\sum_{i=1}^{n}\left(\tilde{\alpha}_{i}-\tilde{\alpha}_{\bullet}\right)^{2}}{\nu+n-1} \\
& \tilde{\alpha}_{\bullet}=\sum_{i=1}^{n} \frac{\tilde{\alpha}_{i}}{n} .
\end{aligned}
$$

As equações dadas em (1.17) são semelhantes às dadas em (1.8) (caso em que $\sigma^{2}$ é conhecida) com $\sigma^{2}$ substituida por $\tilde{\sigma}^{2}$. É interessante notar também que quando $\nu \rightarrow \infty$ as equações dadas em $(1.17)$ se reduzem às dadas em $(1.8) \mathrm{com} \tilde{\sigma}^{2}$ substituida por $\lambda$.

$$
\text { Para se resolver o sistema de equações em (1.17) }
$$
basta utilizar o método de Newton Raphson em (1.9) com 


$$
\begin{aligned}
& c_{i(k)}=\phi_{i} y_{i}-m_{i} \phi_{i} a^{\prime}\left(\alpha_{i(k)}\right)-\frac{\alpha_{i(k)}-\alpha_{\bullet(k)}}{\sigma^{2}{ }_{(k)}}, \\
& i=1, \ldots, n
\end{aligned}
$$

e

$$
\begin{aligned}
C_{i j(k)} & =\left(m_{i} \phi_{i} a^{\prime \prime}\left(\alpha_{i(k)}\right)+\frac{1}{\sigma_{(k)}^{2}}\right) \delta_{i j}-\frac{1}{n \sigma_{(k)}^{2}}- \\
& -2 \frac{\left(\alpha_{i(k)}-\alpha_{\cdot(k)}\right)\left(\alpha_{j(k)}-\alpha_{\cdot(k)}\right)}{\sigma_{(k)}^{4}(\nu+n-1)},
\end{aligned}
$$

$i, j=1, \ldots, n$.

Se assumirmos a aproximação normal da função de den sidade a posteriori, temos que $\mathbf{c}_{(\mathrm{k})}^{-1}$ e $\boldsymbol{\alpha}_{(\mathrm{k})}$ calculados na úl-tima iteração são, respectivamente, a matriz de covariância e o vetor de médias desta densidade. Podemos, portanto, fazer inferências aproximadas sobre o vetor de parâmetros $\boldsymbol{\alpha}$. Leonard (1972) observa que esta aproximação não parece ser muito boa no caso em que $\sigma^{2}$ é desconhecida, porém podemos utilizá-la como um método preliminar por sua simplicidade para inferir sobre $\boldsymbol{\alpha}$. Na Seção 1.3 fornecemos um método mais acurado de estimação aproximada.

Procedendo como na seção anterior, podemos aproximar a função de verossimilhança por uma função de densidade normal multivariada. Neste caso, obtemos a densidade a posteriori aproximada de $\alpha$ proporcional a

$$
\left[\nu \lambda+\sum_{i=1}^{n}\left(\alpha_{i}-\alpha_{\bullet}\right)^{2}\right]^{-\frac{1}{2}(\nu+n-1)} \exp \left\{-\frac{1}{2} \sum_{i=1}^{n} \frac{\left(\alpha_{i}-\hat{\alpha}_{i}\right)^{2}}{v_{i}}\right\} .
$$

E bastante simples encontrar as equações que forne cem a moda a posteriori mas, neste caso em que $\sigma^{2}$ é desconhe 
cida, as equações que levam às estimativas modais não têm sọ luções explícitas.

1.3. Cálculo dos momentos a posteriori aproximados pelo método de Laplace

Tierney e Kadane (1986) descrevem um procedimento para calcular médias, variâncias e covariâncias a posteriori aproximadas de funções positivas e contínuas dos parâmetros. o procedimento, baseado no método de Laplace para integrais, é bastante simples e envolve somente algumas maximizações e derivações. o procedimento está descrito no Apêndice 1.

Seja $\mathrm{g}(\cdot)$ uma função positiva e contínua, definida no espaço paramétrico. A média a posteriori de $g(\theta)$ pode ser aproximada por

$$
\hat{\mathrm{E}}[g(\theta) / y]=\frac{\gamma^{*}}{\gamma} \frac{g\left(\theta^{*}\right) \pi\left(\theta^{*}\right) L\left(\Theta^{*} / \mathrm{y}\right)}{\pi(\tilde{\Theta}) L(\tilde{\Theta} / \mathrm{Y})}
$$

onde $L(\theta / y)$ éa função de verossimilhança, $\pi(\theta)$ é a função de densidade a priori do parâmetro $\theta, y$ é o vetor de observações, Ũé a moda a posteriori, isto é, a moda de $\pi(\theta) L(\theta / y), \quad \theta *$ é a moda de $g(\theta) \pi(\theta) L(\theta / y)$,

$$
\begin{aligned}
& \gamma^{2}=-\left(\frac{d^{2} \log (\pi(\theta) L(\theta / \mathbf{Y}))}{d \Theta^{2}}\right)_{\Theta=\tilde{\Theta}}^{-1} e \\
& \gamma^{*^{2}}=-\left(\frac{d^{2} \log (g(\theta) \pi(\theta) L(\theta / y))}{d \Theta^{2}}\right)_{\theta=\theta^{*}}^{-1} .
\end{aligned}
$$


No caso multivariado, $\theta^{T}=\left(\theta_{1}, \ldots, \theta_{n}\right)$ e

$$
\hat{E}[g(\theta) \dot{y}]=\left(\frac{\operatorname{det} \mathbf{D}^{*}}{\operatorname{det} \mathbf{D}}\right)^{1 / 2} \frac{g\left(\theta^{*}\right) \pi\left(\theta^{*}\right) L\left(\theta^{*} / \mathbf{Y}\right)}{\pi(\tilde{\theta}) L(\tilde{\theta} / \mathbf{Y})}
$$

onde $\tilde{\theta}$ e $\theta *$ são as modas de $\pi(\theta) L(\theta / y)$ e $g(\theta) \pi(\theta) L(\theta / y)$ respectivamente, D é a inversa da matriz cujo elemento $(i, j)$ é

$$
-\left.\frac{\partial^{2} \log (\pi(\theta) L(\theta / y))}{\partial \theta_{i} \partial \theta_{j}}\right|_{\theta=\tilde{\theta}} \quad, i, j=1, \ldots, n
$$

e D* é a inversa da matriz cujo elemento $(i, j)$ é

$$
-\left.\frac{\partial^{2} \log (g(\theta) \pi(\theta) L(\theta / \mathbf{y}))}{\partial \theta_{i} \partial \theta_{j}}\right|_{\theta=\theta^{*}} \quad, i, j=1, \ldots, n \text {. }
$$

Para se obter uma aproximação para a variância de $g(\theta)$ basta tomar

$$
\operatorname{Var}[g(\theta) / \mathbf{Y}]=\hat{E}\left[(g(\theta))^{2} / \mathbf{Y}\right]-\hat{E}^{2}[g(\theta) / \mathbf{y}]
$$

e utilizar a aproximação para a média a posteriori de $g(\theta)$ e de $(g(\theta))^{2}$.

Analogamente, uma aproximação para a covariância de duas funções positivas $g(\theta)$ e $h(\theta)$ é obtida por

$$
\hat{\operatorname{Cov}}[g(\theta), h(\theta) / \mathbf{Y}]=\hat{\mathrm{E}}[g(\boldsymbol{\theta}) \mathrm{h}(\boldsymbol{\theta}) / \mathbf{Y}]-
$$

$$
-\hat{E}[g(\theta) / \mathbf{Y}] \hat{E}[h(\theta) / \mathbf{y}]
$$


A aplicação desta metodologia ao modelo geral estụ dado nas seções anteriores é imediata. o cálculo aproximado dos momentos a posteriori de $\alpha_{i}, i=1, \ldots, n$, não pode ser efetuado pois $\alpha_{i}$ não é exclusivamente positivo. Porém, como foi visto na Seção $1.1, a^{\prime}\left(\alpha_{i}\right)$ é igual à média $\Theta_{i}$ de $Y_{i j}$ dado $\alpha_{i}$ e, além disto, nas distribuições binomial e poisson $a^{\prime}\left(\alpha_{i}\right)$ é positiva. Na seção seguinte passamos a calcular en tão os momentos a posteriori aproximados de a' $\left(\alpha_{i}\right)$, isto é, apresentamos uma aproximação para o estimador de Bayes de $\Theta_{i}$.

1.3.1. Variância da distribuição a priori conhecida

Através de (1.21) podemos calcular a média a poste riori aproximada de $a^{\prime}\left(\alpha_{\ell}\right), \ell=1, \ldots, n$. Para tal, basta to $\operatorname{mar} g(\boldsymbol{\alpha})=a^{\prime}\left(\alpha_{\ell}\right)$ e notar que $\pi(\boldsymbol{\alpha}) L(\boldsymbol{\alpha} / \mathbf{y})$ é proporcional à função de densidade a posteriori marginal de $\boldsymbol{\alpha}$, tendo elimi nado $\mu$ por integração (ver (1.7)). Assim, após alguns cálcu los obtemos a seguinte aproximação para a média a posteriori de $a^{\prime}\left(\alpha_{\ell}\right):$

$$
\begin{gathered}
\hat{E}\left[a^{\prime}\left(\alpha_{\ell}\right) / Y_{1}, \ldots, \mathbf{Y}_{n}, \phi, \sigma^{2}\right]=\left(\frac{\operatorname{det} D^{*}}{\operatorname{det} \mathbf{D}}\right)^{1 / 2} a^{\prime}\left(\alpha_{\ell}^{*}\right) \exp \left\{\sum _ { i = 1 } ^ { n } \left[-\frac{\left(\alpha_{i}^{*}-\alpha^{*}\right)^{2}}{2 \sigma^{2}}+\right.\right. \\
\left.\left.+\frac{\left(\tilde{\alpha}_{i}-\tilde{\alpha}_{\bullet}\right)^{2}}{2 \sigma^{2}}+\phi_{i} y_{i}\left(\alpha_{i}^{*}-\tilde{\alpha}_{i}\right)-m_{i} \phi_{i}\left(a\left(\alpha_{i}^{*}\right)-a\left(\tilde{\alpha}_{i}\right)\right)\right]\right\}, \\
\ell=1, \ldots, n,
\end{gathered}
$$

onde $\tilde{\alpha}_{i}, i=1, \ldots, n$, é obtido através do método de Newton Raphson em (1.9) com $c_{i(k)}$ e $\mathbf{c}_{(\mathrm{k})}^{-1}=\mathbf{D}_{(\mathrm{k})}$ dados por $(1.10)$ e (1.11) respectivamente; $\alpha_{i}^{*}, i=1, \ldots, n$, também é obtido por este método com 


$$
\begin{aligned}
c_{i(k)} & =\phi_{i} Y_{i}-m_{i} \phi_{i} a^{\prime}\left(\alpha_{i(k)}\right)-\frac{\alpha_{i(k)}-\alpha \cdot(k)}{\sigma^{2}}+ \\
& +\frac{\delta_{i \ell^{a}}{ }^{\prime}\left(\alpha_{i(k)}\right)}{a^{\prime}\left(\alpha_{i(k)}\right)}, i=1, \ldots, n
\end{aligned}
$$

e com os elementos de $\mathbf{C}_{(\mathrm{k})}^{-1}=\mathrm{D}_{(\mathrm{k})}^{*}$ dados por

$$
D_{i j(k)}^{*}=w_{i(k)} \delta_{i j}+\frac{w_{i}(k)^{w_{j}(k)}}{n\left(\sigma^{2}-w_{0}(k)\right.}, i, j=1, \ldots, n,
$$

sendo

$$
\begin{aligned}
& w_{i(k)}=\left(\delta_{i \ell} a_{i(k)}+m_{i} \phi_{i} a^{\prime \prime}\left(\alpha_{i(k)}\right)+\frac{1}{\sigma^{2}}\right)^{-1}, \\
& w_{\cdot(k)}=\sum_{i=1}^{n} \frac{w_{i(k)}}{n}
\end{aligned}
$$

e

$$
\begin{aligned}
& a_{i(k)}=-\frac{a^{\prime \prime \prime}\left(\alpha_{i(k)}\right) a^{\prime}\left(\alpha_{i(k)}\right)-\left[a^{\prime}\left(\alpha_{i(k)}\right)\right]^{2}}{\left[a^{\prime}\left(\alpha_{i(k)}\right)\right]^{2}} \text {, } \\
& i=1, \ldots, n
\end{aligned}
$$

e onde D e $\mathbf{D}^{*}$ são, respectivamente, iguais às matrizes $\mathbf{D}_{(\mathrm{k})}$ e $\mathbf{D}_{(\mathrm{k})}^{*}$ obtidas na última iteração das aplicações do método de Newton Raphson.

Convém notar que o cálculo acima envolve derivadas de $a\left(\alpha_{i}\right)$ até terceira ordem, porém, em diversas distribuições, como a binomial e a Poisson estas derivadas existem e são calculadas com facilidade.

Para calcular a covariāncia a posteriori aproximada de a' $\left(\alpha_{\ell}\right)$ e a' $\left(\alpha_{q}\right)$ é necessário calcular a média a posteriori aproximada de produto $a^{\prime}\left(\alpha_{\ell}\right) a^{\prime}\left(\alpha_{q}\right)$ e efetuar o cálculo proposto em (1.23). Se o interesse for calcular a variância de $a^{\prime}\left(\alpha_{\ell}\right)$, basta tomar $\ell=q$, calcular, portanto, a média a 
posteriori aproximada de $\left[a^{\prime}\left(\alpha_{\ell}\right)\right]^{2}$ e fazer o cálculo dado em $(1.22)$.

A média a posteriori aproximada de a' $\left(\alpha_{\ell}\right) a^{\prime}\left(\alpha_{q}\right)^{\prime}$ $\ell, q=1, \ldots, n$, é dada por $(1.24)$ com $a^{\prime}\left(\alpha_{\ell}^{*}\right)$ substituido por $a^{\prime}\left(\alpha_{q}^{*}\right) a^{\prime}\left(\alpha_{q}^{*}\right)$ e $(1.25)$ e $(1.26)$ substituidos, respectivamente, por

$$
\begin{aligned}
& c_{i(k)}=\phi_{i} y_{i}-m_{i} \phi_{i} a^{\prime}\left(\alpha_{i(k)}\right)- \\
&-\frac{\alpha_{i(k)}-\alpha \cdot(k)}{\sigma^{2}}+\frac{\left(\delta_{i \ell}+\delta_{i q}\right) a^{\prime \prime}\left(\alpha_{i(k)}\right)}{a^{\prime}\left(\alpha_{i(k)}\right)}, \\
& i=1, \ldots, n
\end{aligned}
$$

e

$$
\begin{aligned}
& w_{i(k)}=\left[\left(\delta_{i \ell}+\delta_{i q}\right) a_{i(k)}+m_{i} \phi_{i} a^{n}\left(\alpha_{i(k)}\right)+\frac{1}{\sigma^{2}}\right]^{-1}, \\
& i=1, \ldots, n .
\end{aligned}
$$

1.3.2. Variância da distribuição a priori desconhecida

o cálculo dos momentos a posteriori aproximados po de ser efetuado também quando $\sigma^{2}$ é desconhecida. o procedimento é análogo ao utilizado na seção anterior, com a dife rença básica de que se deve calcular a função de densidade a posteriori marginal de $\boldsymbol{\alpha}$ através de integrações com respeito a $\mu$ e $\sigma^{2}$ como foi feito na seção 1.3 (ver $\left.(1.16)\right)$. Após alguns cálculos obtemos a seguinte aproximação para a média a posteriori de $a^{\prime}\left(\alpha_{\ell}\right)$ : 


$$
\begin{array}{r}
\hat{E}\left[a^{\prime}\left(\alpha_{\ell}\right) / y_{1}, \ldots, y_{n}, \theta, \nu, \lambda\right]=\left(\frac{\operatorname{det} D^{*}}{\operatorname{det} \mathbf{D}}\right)^{1 / 2} a^{\prime}\left(\alpha_{\ell}^{*}\right) \times \\
\times \exp \left\{-\frac{1}{2}(\nu+n-1)\left[\log \left[\nu \lambda+\sum_{i=1}^{n}\left(\alpha_{i}^{*}-\alpha_{\bullet}^{*}\right)\right]-\log \left[\nu \lambda+\sum_{i=1}^{n}\left(\tilde{\alpha}_{i}-\dot{\tilde{\alpha}}_{\bullet}\right)^{2}\right]+\right.\right. \\
\left.+\sum_{i=1}^{n}\left[\phi_{i} y_{i}\left(\alpha_{i}^{*}-\tilde{\alpha}_{i}\right)-m_{i} \phi_{i}\left(a\left(\alpha_{i}^{*}\right)-a\left(\tilde{\alpha}_{i}\right)\right)\right]\right\}, \ell=1, \ldots, n,
\end{array}
$$

onde $\tilde{\alpha}_{i}, i=1, \ldots, n$, é obtido através do método de Newton Raphson em (1.9) com $c_{i(k)}$ e $C_{i j(k)}$ dados por (1.19) e (1.20); $\tilde{\alpha}_{i}^{*}, i=1, \ldots, n$, também é obtido pelo mesmo método com

$$
\begin{aligned}
c_{i(k)} & =\phi_{i} y_{i}-m_{i} \phi_{i} a^{\prime}\left(\alpha_{i(k)}\right)-\frac{\alpha_{i(k)}-\alpha \cdot(k)}{\sigma^{2}(k)}+ \\
& +\frac{\delta_{i \ell^{a}}{ }^{\prime \prime}\left(\alpha_{i(k)}\right)}{a^{\prime}\left(\alpha_{i(k)}\right)}, i=1, \ldots, n,
\end{aligned}
$$

e os elementos de $\mathbf{c}_{(\mathrm{k})}$ dados por

$$
\begin{aligned}
C_{i j(k)}=\left(\delta_{i \ell} a_{i(k)}+m_{i} \phi_{i} a^{\prime \prime}\left(\alpha_{i(k)}\right)+\frac{1}{\sigma_{(k)}^{2}}\right) \delta_{i j}-\frac{1}{n_{\sigma^{2}(k)}^{2}}- \\
-\frac{2\left(\alpha_{i(k)}-\alpha \cdot(k)^{)\left(\alpha_{j(k)}-\alpha \cdot(k)\right.}\right)}{\sigma_{(k)}^{4}(v+n-1)}, i, j=1, \ldots, n,
\end{aligned}
$$

com

$$
\sigma_{(k)}^{2}=\frac{v \lambda+\sum_{i=1}^{n}\left(\alpha_{i(k)}-\alpha \cdot(k)\right)^{2}}{v+n-1},
$$

e onde $a_{i(k)}$ é obtido por (1.27) e D e D* são, respectivamente, iguais às matriz’s inversas calculadas na última iteração das aplicações do método de Newton Raphson para o cálculo de $\tilde{\alpha}$ e $\alpha$ *. 
A média a posteriori aproximada de $a^{\prime}\left(\alpha_{\ell}\right) a^{\prime}\left(\alpha_{q}\right)$, ne cessária ao cálculo das variâncias e covariâncias, é dada por (1.28) com a' $\left(\alpha_{\ell}^{*}\right)$ substituido por a' $\left(\alpha_{\ell}^{*}\right) a^{\prime}\left(\alpha_{q}^{*}\right)$ e $(1.29)$ e (1.30) substituidos, respectivamente, por

$$
\begin{aligned}
c_{i(k)} & =\phi_{i} y_{i}-m_{i} \phi_{i} a^{\prime}\left(\alpha_{i(k)}\right)-\frac{\alpha_{i(k)^{\alpha} \cdot(k)}}{\sigma^{2}(k)}+ \\
& +\frac{\left(\delta_{i \ell}+\delta_{i q}\right) a^{n}\left(\alpha_{i(k)}\right)}{a^{\prime}\left(\alpha_{i(k)}\right)}, i=1, \ldots, n
\end{aligned}
$$

e

$$
\begin{aligned}
& c_{i j(k)}=\left[\left(\delta_{i \ell}+\delta_{i q}\right) a_{i(k)}+m_{i} \phi_{i} a^{n}\left(\alpha_{i(k)}\right)+\frac{1}{\sigma_{(k)}^{2}}\right] \delta_{i j}- \\
&-\frac{1}{n_{\sigma_{(k)}^{2}}}-\frac{2\left(\alpha_{i(k)}-\alpha \cdot(k)\right)\left(\alpha_{j(k)}-\alpha \cdot(k)\right.}{\sigma_{(k)}^{4}(v+n-1)}, \\
& i, j=1, \ldots, n .
\end{aligned}
$$

\subsection{Comentários}

Na Seção 1.2 foram propostas duas aproximações para a função de densidade a posteriori de $\boldsymbol{\alpha}$. No entanto, em ge ral, o interesse é fazer inferências sobre $\theta_{i}=a^{\prime}\left(\alpha_{i}\right)$ e não sobre $\alpha_{i}$, uma vez que $\theta_{i}$ é a média de $\mathrm{Y}_{i j}$ dado $\alpha_{i}$. Foi então proposto na seção 1.2. encontrar a moda a posteriori de $\alpha$, denotada por $\tilde{\alpha}^{T}=\left(\tilde{\alpha}_{1}, \ldots, \tilde{\alpha}_{n}\right)$ e estimar $\theta_{i}$ por $\tilde{\theta}_{i}=a^{\prime}\left(\tilde{\alpha}_{i}\right)$. o mesmo raciocínio se aplica para fazer inferências aproximadas sobre $\theta_{i}$. Por exemplo, decerminado um Intervalo de Credí bilidade $\left(\alpha_{i}^{0}, \alpha_{i}^{1}\right)$ para $\alpha_{i}$, pode-se encontrar um Intervado de Credibilidade aproximado para $\Theta_{i}$ da forma $\left(\Theta_{i}^{0}, \Theta_{i}^{1}\right)$ onde $\theta_{i}^{0}=a^{\prime}\left(\alpha_{i}^{0}\right)$ e $\theta_{i}^{1}=a^{\prime}\left(\alpha_{i}^{1}\right)$. Este tipo de procedimento não é 
recomendável pois os erros cometidos não são controlados. Na Seção 1.3. propomos então aproximações mais acuradas sobre os momentos a posteriori de $\mathrm{a}^{\prime}\left(\alpha_{i}\right)$ e, em nossa opinião é melhor trabalhar com estas aproximações do que com as propostas na Seção 1.2. No entanto, não se faz nenhuma aproximação para a função de densidade a posteriori de a' $\left(\alpha_{i}\right)$ e, consequentemen te, não é possível fazer inferências aproximadas sobre $\Theta_{i}$. Po rém o fato de se encontrar aproximações para a média, a variância e a covariância a posteriori já é de grande utilidade na prática.

\subsection{Casos particulares e extensões}

Toda a metodologia descrita nas seções anteriores é baseada na estrutura a priori em dois estágios:

Estágio 1. $\alpha_{i} / \mu, \sigma^{2} \sim N\left(\mu, \sigma^{2}\right), i=1, \ldots, n$, independentes.

Estágio 2. $\pi(\mu) \propto$ constante, $\frac{\nu \lambda}{\sigma^{2}} / \nu, \lambda \sim x_{\nu}^{2}, \nu>0 ; \lambda>0$, onde $\mu$ e $\sigma^{2}$, dados $\nu$ e $\lambda$, são independentes. Foi estudado também o caso em que a va riância $\sigma^{2}$ do primeiro estágio é conhecida. Uma situação tam bém de interesse é aquela em que $\mu$ e $\sigma^{2}$ são ambas conhecidas ou que $\mu$ é conhecida e $\sigma^{2}$ é desconhecida. Ou ainda pode-se pensar numa distribuição informativa para $\mu$ do tipo: 


$$
\mu / \mu_{0}, \sigma_{0}^{2} \sim N\left(\mu_{0}, \sigma_{0}^{2}\right)
$$

com, digamos, $\mu_{0}$ conhecido e $\sigma_{0}^{2}$ desconhecida com a seguinte distribuição a priori:

$$
\frac{\nu_{0} \lambda_{0}}{\sigma_{0}^{2}} / \nu_{0}, \lambda_{0} \sim x_{\nu_{0}}^{2}, \nu_{0}>0, \lambda_{0}>0 .
$$

Os resultados para estas situações são obtidos de forma absolutamente análoga à descrita nas seções anteriores e, portanto, serão omitidos.

OOO 


\section{CAPITULO 2}

\section{ALGUNS MODELOS ESPECIAIS}

\section{1. o modelo binomial}

Consideremos n populações das quais são retiradas amostras aleatórias independentes de tamanhos $m_{1}, \ldots, m_{n}$ respectivamente. Suponhamos que cada variável aleatória $Y_{i j}$ ' $i=1, \ldots, n, j \doteq 1, \ldots, m_{i}$, dado $\theta_{i}$, tenha distribuição de $m_{i}$ Bernoulli com probabilidade de sucesso $\theta_{i}$. Logo, $Y_{i}=\sum_{j=1}^{i} Y_{i j}$, dados $m_{i} e \theta_{i}$, tem distribuição binomial com índice $m_{i}$ e pro babilidade de sucesso $\theta_{i}$, que denotamos por

$$
Y_{i} / m_{i}, \theta_{i} \sim B\left(m_{i}, \theta_{i}\right), \quad i=1, \ldots, n \text {. }
$$

Um estudo sobre estimação Bayesiana neste modelo é encontrada em Leonard (1972) e Leonard (1976).

A função de probabilidade de $\mathrm{Y}_{i}$ é um caso particular de $(1.2) \operatorname{com} \alpha_{i}=\log \left(\Theta_{i} /\left(1-\theta_{i}\right)\right), 0<\theta_{i}<1$ (ou, equivalentemente, $\left.\theta_{i}=e^{\alpha_{i}} /\left(1+e^{\alpha_{i}}\right)\right), a\left(\alpha_{i}\right)=\log \left(1+e^{\alpha_{i}}\right)$ e $\phi_{i}=1$, $i=1, \ldots, n$ levidentemente, $y_{i}$ é um inteiro compreendido entre zero e $\mathrm{m}_{i}$ ). Logo, de (1.3), temos que a função de verossimi lhança de $\boldsymbol{\alpha}$ é

$$
L\left(\alpha / y_{1}, \ldots, y_{n}\right) \propto \exp \left\{\sum_{i=1}^{n}\left[y_{i} \alpha_{i}-m_{i} \log \left(1+e^{\alpha_{i}}\right)\right]\right\} .
$$


2.1.1. Variância da distribuição a priori conhecida

Consideremos a estrutura a priori estabelecida em (1.4) e (1.5). Na análise de dados binomiais é comum a uti-lização de uma distribuição a priori beta para $\Theta_{i}$. Portanto, é razoável utilizar uma distribuição a priori normal para os $\alpha_{i}$ 's uma vez que se $\alpha_{i}$ tem distribuição normal então $\theta_{i}=e^{\alpha} /\left(1+e^{\alpha}\right)$ tem aproximadamente distribuição beta (Leonard, 1972).

Por (1.7) temos que a função de densidade a poste riori de $\boldsymbol{\alpha}$ é

$$
\pi\left(\alpha / y_{1}, \ldots, y_{m}, \sigma^{2}\right) \propto \exp \left\{\sum_{i=1}^{n}\left[y_{i} \alpha_{i}-m_{i} \log \left(1+e^{\alpha_{i}}\right)-\frac{1}{2 \sigma^{2}}\left(\alpha_{i}-\alpha_{\bullet}\right)^{2}\right]\right\}
$$

e de (1.8) segue que as modas a posteriori são dadas pela solução do sistema de equações:

$$
\frac{e^{\tilde{\alpha}_{i}}}{1+e^{\tilde{\alpha}_{i}}}=\frac{y_{i}}{m_{i}}-\frac{\tilde{\alpha}_{i}-\tilde{\alpha}}{m_{i} \sigma^{2}}, i=1, \ldots, n
$$

Como $\theta_{i}=e^{\alpha_{i}} /\left(1+e^{\alpha_{i}}\right)$, um estimador natural de $\theta_{i}$ é $\tilde{\theta}_{i}=e^{\tilde{\alpha}_{i}} /\left(1+e^{\tilde{\alpha}_{i}}\right)$.

Para encontrar a solução de (2.1) pode-se utilizar o método de Newton Raphson em (1.9), bastando tomar

$$
c_{i(k)}=y_{i}-m_{i} \frac{e^{\alpha_{i}(k)}}{1+e^{\alpha_{i(k)}}}-\frac{\alpha_{i(k)}-\alpha \cdot(k)}{\sigma^{2}}, i=1, \ldots, n(2.2)
$$

e o elemento $(i, j)$ da matriz $\mathbf{c}_{(k)}^{-1}$ igual a 


$$
D_{i j(k)}=u_{i(k)} \delta_{i j}+\frac{u_{i(k)} u_{j(k)}}{n\left(\sigma^{2}-u_{0}(k)\right.}, i, j=1, \ldots, n
$$

onde

$$
u_{i(k)}=\left(m_{i} \frac{e^{\alpha_{i}(k)}}{\left(1+e^{\alpha_{i}(k)}\right)^{2}}+\frac{1}{\sigma^{2}}\right)^{-1}, i=1, \ldots, n .
$$

Como na Seção 1.2.1 podemos aproximar a função de densidade a posteriori por uma normal multivariada com vetor de médias $\alpha_{(k)}$ e matriz de covariância $\mathbf{c}_{(\mathrm{k})}^{-1}$ obtidos na última iteração do processo de Newton Raphson. Na opinião de Leonard (1972) esta aproximação é razoavelmente acurada numa grande região do espaço paramétrico desde que $y_{i}$ e $m_{i}-y_{i}$ não sejam pequenos.

Agora, assumindo uma aproximação normal para a fun ção de verossimilhança temos, por (1.12) que as médias a pos teriori aproximadas são

$$
\tilde{\alpha}_{i}^{*}=\frac{\frac{\hat{\alpha}_{i}}{v_{i}}+\frac{\hat{\alpha}_{0}}{\sigma^{2}}}{\frac{1}{v_{i}}+\frac{1}{\sigma^{2}}}, i=1, \ldots, n,
$$

onde

$$
\begin{aligned}
& \hat{\alpha}_{i}=\log y_{i}-\log \left(m_{i}-y_{i}\right), i=1, \ldots, n, \\
& v_{i}=\frac{1}{y_{i}}+\frac{1}{m_{i}-y_{i}}, i=1, \ldots, n
\end{aligned}
$$

e

$$
\hat{\alpha}_{0}=\frac{\sum_{i=1}^{n} \frac{\hat{\alpha}_{i}}{\sigma^{2}+v_{i}}}{\sum_{i=1}^{n} \frac{1}{\sigma^{2}+v_{i}}} .
$$


Por (1.13) pode-se encontrar a matriz de covariância da distribuição a posteriori aproximada e fazer inferências aproximadas sobre o vetor de parâmetros $\boldsymbol{\alpha}$.

2.1.2. Variância da distribuição a priori desconhecida.

Consideremos agora a estrutura a priori (1.14) e (1.15). Neste caso, a função de densidade a posteriori de $\boldsymbol{\alpha}$ é, por $(1.16)$,

$$
\begin{aligned}
\pi\left(\alpha / y_{1}, \ldots, y_{n}, \nu, \lambda\right) \propto\left[\nu \lambda+\sum_{i=1}^{n}\left(\alpha_{i}-\alpha_{\bullet}\right)^{2}\right]^{-\frac{1}{2}(\nu+n-1)} \times \\
\times \exp \left\{\sum_{i=1}^{n}\left[y_{i} \alpha_{i}-m_{i} \log \left(1+e^{\alpha_{i}}\right)\right]\right\} .
\end{aligned}
$$

Por $(1.17)$ e $(1.18)$, as modas a posteriori são obtid̄as pela solução do sistema de equações dado em (2.1) com $\sigma^{2}$ substituida por $\tilde{\sigma}^{2}$.

É possível utilizar o mesmo processo iterativo pro posto em (1.9) para encontrar as modas a posteriori, bastando substituir $c_{i(k)}$ e $C_{i j(k)}$,respectivamente, por

$$
y_{i}-m_{i} \frac{e^{\alpha_{i(k)}}}{1+e^{\alpha_{i(k)}}}-\frac{\alpha_{i(k)}-\alpha,(k)}{\sigma^{2}(k)}, i=1, \ldots, n \text { (2.5) }
$$

e

$$
\begin{aligned}
& \left(m_{i} \frac{e^{\alpha} i(k)}{\left.\alpha_{i(k)}\right)^{2}}+\frac{1}{\sigma_{(k)}^{2}}\right) \delta_{i j}-\frac{1}{n \sigma_{(k)}^{2}}-\frac{2\left(\alpha_{i(k)}-\alpha \cdot(k)\right)\left(\alpha_{j(k)}-\alpha \cdot(k)\right.}{\sigma_{(k)}^{4}(v+n-1)} \quad(2.6) \\
& i, j=1, \ldots, n
\end{aligned}
$$


As aproximações propostas na Seção 1.2 .2 podem ser também facilmente aplicadas ao caso de dados binomiais.

2.1.3. Cálculo dos momentos aproximados pelo método de Laplace

Na distribuição binomial, a média de $Y_{i j}$ dado $\alpha_{i}$ é $e^{\alpha_{i}} /\left(1+e^{\alpha}{ }^{\alpha}\right)$. Esta é uma função positiva e contínua de $\alpha_{i}$, portanto, o procedimento do cálculo dos momentos a posteriori aproximados pelo método de Laplace pode ser usado. o cálculo destes momentos será omitido aqui. Para obtê-los basta lembrar que $a\left(\alpha_{i}\right)=\log \left(1+e^{\alpha}\right)$ e $\phi_{i}=1, i=1, \ldots$, n e utilizar os resultados das seções 1.3 .1 e e 1.3.2.

\section{2. o modelo de Poisson}

Consideremos $\mathrm{n}$ populações das quais são retiradas amostras aleatórias independentes $\mathbf{Y}_{1}, \ldots, \mathbf{Y}_{\mathrm{n}}$ de tamanhos $m_{1}, \ldots, m_{n}$ respectivamente. Suponhamos que, dado $\theta_{i}, Y_{i j}$ tenha distribuição de Poisson com média $\Theta_{i}$ que denotamos por:

$$
Y_{i j} / \Theta_{i} \sim P\left(\Theta_{i}\right), i=1, \ldots, n, j=1, \ldots, m_{i} .
$$

Um estudo sobre estimação Bayesiana neste modelo é encontrada em Leonard (1976).

A função de probabilidade de $\mathrm{Y}_{i j}$ é um caso parti cular de (1.2) $\operatorname{com} \alpha_{i}=\log \Theta_{i}$ (ou, equivalentemente, $\theta_{i}=e^{\alpha}{ }^{\alpha}$, , $\phi_{i}=1 \mathrm{e} a\left(\alpha_{i}\right)=e^{\alpha_{i}}, i=1, \ldots, n$ (e, evidentemente, $y_{i}$ é um inteiro não negativo). 
De (1.3) temos que a função de verossimilhança de $\boldsymbol{\alpha}$ é

$$
L\left(\alpha / y_{1}, \ldots, y_{n}\right) \propto \exp \left\{\sum_{i=1}^{n}\left(y_{i} \alpha_{i}-m_{i} e^{\alpha}\right)\right\}
$$

onde

$$
y_{i}=\sum_{i=1}^{n} y_{i j}, i=1, \ldots, n
$$

2.2.1. Variância da distribuição a priori conhecida

Tomemos a estrutura a priori dada em (1.4) e (1.5). Note-se que estamos assumindo uma distribuição a priori lognormal para $\theta_{i}$.

De (1.7) segue que a função de densidade a poste riori de a é

$$
\pi\left(\alpha / y_{1}, \ldots, y_{n}, \sigma^{2}\right) \propto \exp \left\{\sum_{i=1}^{n}\left(y_{i} \alpha_{i}-m_{i} e^{\alpha}\right)-\frac{1}{2 \sigma^{2}} \sum_{i=1}^{n}\left(\alpha_{i}-\alpha_{0}\right)^{2}\right\}
$$

e de (1.8) que as modas a posteriori são dadas pela solução do sistema de equações

$$
e^{\tilde{\alpha}_{i}}=\frac{y_{i}}{m_{i}}-\frac{\tilde{\alpha}_{i}-\tilde{\alpha}}{m_{i} \sigma^{2}}, i=1, \ldots, n
$$

Como $\theta_{i}=e^{\alpha_{i}}$, um estimador natural de $\theta_{i} \bar{e} \tilde{\theta}_{i}=e^{\tilde{\alpha}_{i}}$.

O método de Newton Raphson em (1.9) pode ser usado para encontrar a solução de (2.7) bastando tomar

$$
c_{i(k)}=y_{i}-m_{i} e^{\alpha_{i}(k)}-\frac{\alpha_{i(k)}-\alpha \cdot(k)}{\sigma^{2}}, i=1, \ldots, n
$$


e o elemento $(i, j)$ da matriz $c_{(k)}^{-1}$ igual a

onde

$$
D_{i j(k)}=u_{i(k)} \delta_{i j}+\frac{u_{i(k)} u_{j(k)}}{n\left(\sigma^{2}-u_{.(k)}\right)}, i, j=1, \ldots, n
$$

$$
u_{i(k)}=\left(m_{i} e^{\alpha_{i}(k)}+\frac{1}{\sigma^{2}}\right)^{-1} \quad, i=1, \ldots, n .
$$

Como foi citado na Seção 1.2.1, é possivel aproximar a função de densidade a posteriori por uma normal multiva riada com vetor de médias $\alpha_{(k)}$ e matriz de covariância $c_{(k)}^{-1}$ obtidos na última iteração do processo de Newton Raphson.

Se assumirmos uma aproximação normal para a função de verossimilhança, segue de (1.12) que as médias a posterioria aproximadas são

$$
\tilde{\alpha}_{i}^{*}=\frac{\frac{\hat{\alpha}_{i}}{v_{i}}+\frac{\hat{\alpha}}{\sigma^{2}}}{\frac{1}{v_{i}}+\frac{1}{\sigma^{2}}}, i=1, \ldots, n,
$$

onde $\hat{\alpha}_{i}=\log y_{i}-\log m_{i}, i=1, \ldots, n$,

$$
v_{i}=\frac{1}{y_{i}}, i=1, \ldots, n
$$

e

$$
\hat{\alpha}_{.}=\frac{\sum_{i=1}^{n} \frac{\hat{\alpha}_{i}}{\sigma^{2}+v_{i}}}{\sum_{i=1}^{n} \frac{1}{\sigma^{2}+v_{i}}}
$$

Finalmente, por (1.13) pode-se encontrar a matriz de covariância da distribuição a posteriori aproximada e fazer inferências aproximadas sobre o vetor de parâmetros $\boldsymbol{\alpha}$. 
2.2.2. Variância da distribuição a priori desconhecida

Tomando a estrutura a priori em (1.14) e (1.15) quando $\sigma^{2}$ é desconhecida temos, por (1.16), que a função de densidade a posteriori de $\boldsymbol{\alpha}$ é

$$
\begin{aligned}
\pi\left(\alpha / y_{1}, \ldots, \mathbf{y}_{n}, \nu, \lambda\right) & \propto\left[\nu \lambda+\sum_{i=1}^{n}\left(\alpha_{i}-\alpha_{0}\right)^{2}\right]^{-\frac{1}{2}(\nu+n-1)} \times \\
& \times \exp \left\{\sum_{i=1}^{n}\left(y_{i} \alpha_{i}-m_{i} e^{\alpha_{i}}\right)\right\} .
\end{aligned}
$$

De (1.17) e (1.18) segue que as modas a posteriori são obtidas pela solução do sistema de equações dado em (2.7) com $\sigma^{2}$ substituida por $\tilde{\sigma}^{2}$.

O processo iterativo em (1.9) é utilizado com $\mathrm{c}_{i(\mathrm{k})}$ e $C_{i j(k)}$ substituidos, respectivamente, por

$$
y_{i}-m_{i} e^{\alpha_{i}(k)}-\frac{\alpha_{i(k)}-\alpha \cdot(k)}{\sigma_{(k)}^{2}}
$$

e

$$
\begin{aligned}
& \left(m_{i} e^{\alpha_{i}(k)}+\frac{1}{\sigma_{(k)}^{2}}\right) \delta_{i j}-\frac{1}{n \sigma_{(k)}^{2}}-\frac{2\left(\alpha_{i(k)}-\alpha_{\cdot(k)}\right)\left(\alpha_{j(k)}-\alpha_{\bullet(k)}\right)}{\sigma_{(k)}^{4}(\nu+n-1)}, \\
& i, j=1, \ldots, n .
\end{aligned}
$$

As aproximações propostas na Seção 1.2.2. podem ser também aplicadas a নados provindos da distribuição de Poisson. 
2.2.3. Cálculo dos momentos a posteriori aproximados pelo método de Laplace

A média de $Y_{i j}$ dado $\alpha_{i}$ é, na distribuição de Poisson, igual a $e^{\alpha_{i}}$. Como esta é uma função positiva de $\alpha_{i}$, o procedimento do cálculo dos momentos aproximados pelo método de Laplace pode ser utilizado. Para obter estes momentos basta lembrar que $a\left(\alpha_{i}\right)=e^{\alpha_{i}}$ e $\phi_{i}=1, i=1, \ldots, n$ e utilizar os resultados das seções 1.3 .1 e 1.3 .2 .

\section{3. o modelo normal}

Consideremos $\mathrm{n}$ populações das quais são retiradas amostras aleatórias independentes $\mathrm{Y}_{1}, \ldots, \mathrm{Y}_{\mathrm{n}}$ de tamanhos $m_{1}, \ldots, m_{n}$ respectivamente. Suponhamos que, dados $\theta_{i}$ e $\sigma_{i}^{2}$, $Y_{i j}$ tenha distribuição normal com média $\Theta_{i}$ e variância $\sigma_{i}^{2}$ (as sumida, a princípio, conhecidal que denotamos por

$$
Y_{i j} / \Theta_{i}, \sigma_{i}^{2} \sim N\left(\Theta_{i}, \sigma_{i}^{2}\right), i=1, \ldots, n, j=1, \ldots, m_{i} .
$$

A função de densidade de $Y_{i j}$, dados $\theta_{i}$ e $\sigma_{i}^{2}$, é um caso particular de (1.2) com $\alpha_{i}=\theta_{i}, \phi_{i}=1 / \sigma_{i}^{2}$ e $a\left(\alpha_{i}\right)=\alpha_{i}^{2} / 2$. De (1.3) segue que a função de verossimilhança de $\theta$ (lembrando que $\left.\alpha_{i}=\theta_{i}, i=1, \ldots, n\right)$ é

$$
L\left(\theta \mid y_{1}, \ldots, y_{n}\right) \propto \exp \left\{\sum_{i=1}^{n} \frac{1}{\sigma_{i}^{2}}\left(y_{i} \Theta_{i}-m_{i} \frac{\Theta_{i}^{2}}{2}\right)\right\}
$$

onde $y_{i}=\sum_{j=1}^{m_{i}} y_{i j}, i=1, \ldots, n$. 


\subsubsection{Variâncias conhecidas}

Vamos assumir, nesta seção, que as variâncias $\sigma^{2} \mathrm{e}$ $\sigma_{i}^{2}, i=1, \ldots, n$, são todas conhecidas. Tomemos a estrutura a priori dada em (1.4) e (1.5). Como $\alpha_{i}=\theta_{i}, i=1, \ldots, n$, estamos assumindo uma distribuição a priori normal para $\Theta_{i}$.

Por (1.7) temos que a função de densidade a posteriori de $\theta$ é

$$
\pi\left(\theta \mid \mathbf{y}_{1}, \ldots, \mathbf{y}_{n}, \sigma_{1}^{2}, \ldots, \sigma_{n}^{2}, \sigma^{2}\right) \propto \exp \left\{\sum_{i=1}^{n} \frac{1}{\sigma_{i}^{2}}\left(y_{i} \Theta_{i}-m_{i} \frac{\Theta_{i}^{2}}{2}\right)-\frac{1}{2 \sigma^{2}} \sum_{i=1}^{n}\left(\Theta_{i}-\Theta_{0}\right)^{2}\right\}
$$

onde $\theta_{\bullet}=\sum_{i=1}^{n} \frac{\theta_{i}}{n}$. Esta é uma função de densidade normal mul tivariada, logo as modas e as médias a posteriori coincidem. Podemos, portanto, encontrar os estimadores de Bayes de $\Theta_{i}$, $i=1, \ldots, n$ (em relação à perda quadrática) já que estes são dados pelas médias da distribuição a posteriori. De (1.8) segue que

$$
\tilde{\Theta}_{i}=\frac{y_{i}}{m_{i}}-\sigma_{i}^{2} \frac{\tilde{\Theta}_{i}-\tilde{\Theta}_{i}}{m_{i} \sigma^{2}}, i=1, \ldots, n,
$$

onde

$$
\tilde{\Theta}=\sum_{i=1}^{n} \frac{\tilde{\Theta}_{i}}{n}
$$

É fácil mostrar que

$$
\tilde{\Theta} .=\frac{\sum_{i=1}^{n} \frac{y_{i}}{\sigma^{2}+\sigma_{i}^{2} / m_{i}}}{\sum_{i=1}^{n} \frac{1}{\sigma^{2}+\sigma_{i}^{2} / m_{i}}},
$$


onde $\bar{y}_{i}=y_{i} / m_{i}$. L Logo, $\tilde{\Theta}$. é uma média ponderada das médias das amostras com pesos iguais aos inversos de $\sigma^{2}+\sigma_{i}^{2} / m_{i}$ outra forma de escrever $\tilde{\Theta}_{i}$ é

$$
\tilde{\theta}_{i}=d_{i} \bar{y}_{i}+\left(1-d_{i}\right) \tilde{\Theta}, \quad, \quad i=1, \ldots, n,
$$

onde $d_{i}=\sigma^{2} /\left(\sigma^{2}+\sigma_{i}^{2} / m_{i}\right)$. Isto mostra uma contração em direção a $\tilde{\Theta}_{.}$, que será tanto maior quanto menor for $d_{i}$, o que ocorre quando $\sigma^{2}$ é pequena quando comparada com $\sigma_{i}^{2} / \mathrm{m}_{i}$.

A matriz de covariância a posteriori D, por (1.11), tem elementos dados por

$$
D_{i j}=u_{i} \delta_{i j}+\frac{u_{i} u_{j}}{n\left(\sigma^{2}-u_{0}\right)}, i, j=1, \ldots, n,
$$

onde

$$
u_{i}=\left(\frac{m_{i}}{\sigma_{i}^{2}}+\frac{1}{\sigma^{2}}\right)^{-1} .
$$

As aproximações feitas na Seção 1.2 .1 não são necessárias aqui, uma vez que a distribuição a posteriori é normal multivariada.

\subsubsection{Variâncias desconhecidas}

Na seção anterior, supusemos $\sigma_{i}^{2}, i=1, \ldots, n$ e $\sigma^{2}$ conhecidas. Podemos supor apenas $\sigma^{2}$ desconnecida e proceder a análise como na seção 1.2 .2 porém, na prática, é comum não se conhecer também $\sigma_{i}^{2}$. Nesta situação, vamos assumir a se.guinte estrutura a priori: 
Estágio 1. $\Theta_{i} \mid \mu, \sigma^{2} \sim N\left(\mu, \sigma^{2}\right), i=1, \ldots, n$, independentes.

Estágio 2. $\pi(\mu) \propto$ constante,

$$
\begin{aligned}
& \frac{v \lambda}{\sigma^{2}} / \nu, \lambda \sim x_{\nu}^{2}, \nu>0, \lambda>0, \\
& \frac{\nu_{i} \lambda}{\sigma_{i}^{2}} / \nu_{i}, \lambda_{i} \sim x_{\nu_{i}}^{2}, \nu_{i}>0, \lambda_{i}>0, i=1, \ldots, n,
\end{aligned}
$$

onde, dados $\nu, \lambda, \nu_{i}, \lambda_{i}, i=1, \ldots, n, \mu, \sigma^{2}$ e $\sigma_{i}^{2}, i=1, \ldots, n$, são independentes.

Este assunto é tratado por Lindley e Smith (1972). Eles propõem que seja calculada a função de densidade conjunta de todas as quantidades desconhecidas e, em lugar de eliminar as variâncias $\sigma^{2}$ e $\sigma_{i}^{2}, i=1, \ldots, n$, por integração, encontrar uma moda conjunta $\tilde{\sigma}^{2}, \tilde{\sigma}_{i}^{2}, \tilde{\Theta}_{i}, i=1, \ldots, n$. Não utiliza mos este procedimento pois a integração com respeito às vari âncias é bastante simples de ser efetuada e portanto, encontrar a moda conjunta em lugar da marginal de $\theta$, é uma aproximação desnecessária. A análise como fazemos nesta seção é encontrada em Lindley (1971).

A função de densidade a posteriori conjunta de $\theta, \mu, \sigma^{2}, \sigma_{1}^{2}, \ldots, \sigma_{n}^{2}$ é dada por

$$
\begin{aligned}
& \pi\left(\theta, \mu, \sigma^{2}, \sigma_{1}^{2}, \ldots, \sigma_{n}^{2} / \mathbf{y}_{1}, \ldots, y_{n}, \nu, \lambda\right) \propto \\
& \propto L\left(\theta, \mu, \sigma_{1}^{2}, \ldots, \sigma_{n}^{2}, \sigma^{2} / y_{1}, \ldots, y_{n}\right) \quad \times \\
& \times \pi\left(\theta / \mu, \sigma^{2}\right) \pi(\mu) \pi\left(\sigma^{2} / \nu, \lambda\right) \prod_{i=1}^{n} \pi\left(\sigma_{i}^{2} / \nu_{i}, \lambda{ }_{i}\right)
\end{aligned}
$$




$$
\begin{aligned}
& \propto \prod_{i=1}^{n}\left(\sigma_{i}^{2}\right)^{-\frac{1}{2}\left(\nu_{i}+m_{i}+2\right)}\left(\sigma^{2}\right)^{-\frac{1}{2}(\nu+n+2)} \times \\
& \times \exp \left\{-\sum_{i=1}^{n} \frac{\nu_{i} \lambda_{i}+S_{i}^{2}+m_{i}\left(\bar{y}_{i}-\theta_{i}\right)^{2}}{2 \sigma_{i}^{2}}-\frac{\nu \lambda+\sum_{i=1}^{n}\left(\theta_{i}-\mu\right)^{2}}{2 \sigma^{2}}\right\}
\end{aligned}
$$

onde $s_{i}^{2}=\sum_{j=1}^{m_{i}}\left(y_{i j}-\bar{y}_{i}\right)^{2}, i=1, \ldots, n, v^{T}=\left(\nu, \nu_{1}, \ldots, \nu_{n}\right)$ e $\lambda^{T}=\left(\lambda, \lambda_{1}, \ldots, \lambda_{n}\right)$.

A integração com respeito a $\mu$ produz

$$
\left.\begin{array}{l}
\pi\left(\boldsymbol{\theta}, \sigma_{1}^{2}, \ldots, \sigma_{n}^{2}, \sigma^{2} / \mathbf{y}_{1}, \ldots, \mathbf{Y}_{n}, \boldsymbol{\nu}, \boldsymbol{\lambda}\right) \propto \\
\prod_{i=1}^{n}\left(\sigma_{i}^{2}\right) \\
\times \exp \left\{-\sum_{i=1}^{-\frac{1}{2}\left(\nu_{i}+m_{i}+2\right)}\left(\sigma_{i}^{2}\right)^{-\frac{1}{2}(\nu+n+1) .}\right. \\
2 \sigma_{i}^{2}
\end{array}\right\} .
$$

A integração com respeito a $\sigma^{2}, \sigma_{1}^{2}, \ldots, \sigma_{n}^{2}$ fornece a função de densidade a posteriori marginal de $\theta$ que é dada por

$$
\begin{aligned}
\pi\left(\theta \mid y_{1}, \ldots, y_{n}\right) & \propto\left[\nu \lambda+\sum_{i=1}^{n}\left(\theta_{i}-\theta_{0}\right)^{2}\right]^{-\frac{1}{2}(\nu+n-1)} \times \\
& \times \prod_{i=1}^{n}\left[\nu_{i} \lambda_{i}+S_{i}^{2}+m_{i}\left(\bar{y}_{i}-\theta_{i}\right)^{2}\right]^{-\frac{1}{2}\left(\nu_{i}+m_{i}\right)}
\end{aligned}
$$

Este é o produto de funções de densidade $t$ multivariadas. Como já citado, não se pode tomar $v=0$ pois, neste caso, a distribuição a posteriori não é própria. No 
entanto,é possível tomar $\nu_{1}, \ldots, v_{n}$ iguais a zero, correspondendo a prioris não informativas para $\sigma_{1}^{2}, \ldots, \sigma_{n}^{2}$. Assim, não é necessário especificar valores para $\lambda_{1}, \ldots, \lambda_{n}$.

Agora, basta maximizar a função acima com relação a $\theta$ para encontrar a moda a posteriori. É fácil mostrar que ela é dada por

$$
\tilde{\Theta}_{i}=\tilde{d}_{i} \bar{y}_{i}+\left(1-\tilde{d}_{i}\right) \tilde{\theta} \text {. }
$$

onde

$$
\begin{aligned}
& \tilde{d}_{i}=\frac{\tilde{\sigma}^{2}}{\tilde{\sigma}^{2}+\tilde{\sigma}_{i}^{2} / m_{i}}, i=1, \ldots, n, \\
& \tilde{\sigma}^{2}=\frac{\nu \lambda+\sum_{i=1}^{n}\left(\tilde{\Theta}_{i}-\tilde{\Theta}_{0}\right)^{2}}{\nu+n-1}, \tilde{\sigma}_{i}^{2}=\frac{\nu_{i} \lambda_{i}+s_{i}^{2}+m_{i}\left(\bar{y}_{i}-\tilde{\Theta}_{i}\right)^{2}}{\nu_{i}+m_{i}}, \\
& i=1, \ldots, n,
\end{aligned}
$$

e

$$
\tilde{\Theta}_{.}=\frac{\sum_{i=1}^{n} \frac{\bar{y}_{i}}{\tilde{\sigma}^{2}+\tilde{\sigma}_{i}^{2} / m_{i}}}{\sum_{i=1}^{n} \frac{1}{\tilde{\sigma}^{2}+\tilde{\sigma}_{i}^{2} / m_{i}}} .
$$

Note-se que os estimadores modais obtidos aqui são semelhantes àqueles em que se supunham conhecidas as variâncias, porém, com $\sigma^{2}, \sigma_{1}^{2}, \ldots, \sigma_{n}^{2}$ substituidas por $\tilde{\sigma}^{2}, \tilde{\sigma}_{1}^{2}, \ldots, \tilde{\sigma}_{n}^{2}$ respectivamente.

A solução de $(2.10)$ pode ser obtida pelo método de Newton Raphson descrito na Seção 1.2.1, o que implica na so lução iterativa de (1.9) com $\alpha_{i(k)}, C_{i j(k)}$ e $c_{i(k)}$ substi tuidos, respectivamente, por $\Theta_{i(k)}$, 


$$
c_{i(k)}=\frac{y_{i}}{\sigma_{i(k)}^{2}}-\frac{m_{i} \theta_{i(k)}}{\sigma_{i(k)}^{2}}-\frac{\theta_{i(k)}-\theta \cdot(k)}{\sigma_{(k)}^{2}}, i=1, \ldots, n
$$

e

$$
\begin{aligned}
& C_{i j(k)}=\left(\frac{m_{i}}{\sigma_{i(k)}^{2}}-\frac{2 m_{i}^{2}\left(\bar{y}_{i}-\Theta_{i}(k)\right)^{2}}{\left(\nu_{i}+m_{i}\right) \sigma_{i(k)}^{4}}+\frac{1}{\sigma_{(k)}^{2}}\right) \delta_{i j}- \\
& \left.-\frac{1}{n \sigma^{2}(k)}-2 \frac{\left({ }^{\Theta} i(k)^{-\theta} \cdot(k)^{(\theta} j(k)^{-\theta} \cdot(k)\right.}{}\right) \\
& i, j=1, \ldots, n \text {. }
\end{aligned}
$$

As aproximações propostas na seção 1.2 .2 podem ser facilmente aplicadas aqui, isto é, ao problema de dados normais agrupados.

Uma situação bastante comum na prática é aquela em que $\sigma_{1}^{2}=\ldots=\sigma_{n}^{2}$, isto é, assume-se a priori que as variâncias "dentro" das populações são iguais. Comumente esta vari ância é denotada por $\sigma_{\mathrm{W}}^{2}$ (within variance) e a que chamamos de $\sigma^{2}$ é denotada por $\sigma_{\mathrm{b}}^{2}$ (between variance). A análise desta sí tuação é semelhante à que fizemos acima e portanto será omitị da.

\subsubsection{Cálculo dos momentos a posteriori aproximados pelo método de Laplace}

Na Seção 2.3.1 ficou claro que no caso em que as variâncias são conhecidas não há nece;sidade de se fazer aprọ ximações para a função de densidade a posteriori pois mostramos que esta é normal multivariada, porém, quando as variâncias são desconhecidas estas aproximações são necessárias. 
Infelizmente, o procedimento cálculo dos momentos a posterio ri aproximados pelo método de Laplace proposto por Tierney e Kadane (1986) só é válido para funções positivas dos parâmetros. Na situação presente, $\Theta_{i}$ pode ser negativa e consequen temente não se pode utilizar tal procedimento. Uma solução é, portanto, utilizar o estimador modal proposto na seção anterior. Outra solução para o problema é dada em Andrade e Bolfarine (1986). 
CAPITULO 3

PREVISÃO BAYESIANA EM AMOSTRAGEM EM DOIS ESTÁGIOS

\subsection{Introdução}

3.1.1. O enfoque de superpopulação para populações finitas

Uma população finita consiste de $\mathrm{N}$ unidades rotuladas pelos inteiros $1, \ldots, N$. À i-ésima unidade da população está associado um valor desconhecido $y_{i}$. O enfoque de superpopulação assume que $\mathrm{y}_{1}, \ldots, \mathrm{y}_{\mathrm{N}}$ são realizações das variáveis aleatórias $\mathrm{Y}_{1}, \ldots, \mathrm{Y}_{\mathrm{N}}$ respectivamente, com uma certa distribui ção de probabilidades conjunta. Como $\mathrm{y}_{1}, \ldots, \mathrm{y}_{\mathrm{N}}$ são desconhecidos, é de interesse prever funções destas quantidades como, por exemplo, o total populacional $\sum_{i=1}^{N} y_{i}$ e a média populacional $\sum_{i=1}^{N} y_{i} / N$

Sobre a teoria geral da previsão em populações finitas citamos a seguinte bibliografia: Rodrigues e Bolfarine (1984), Cassel, Sarndal e Wretman (1977), Rodrigues, Bolfarine e Rogatko (1985), Sandoval (1987) e Elian (1984), os dois últimos tratando somente de previsão linear. 
3.1.2. Amostragem em dois estágios

Para trabalhar com amostragem em dois estágios é necessário utilizar uma notação distinta da acima mencionada. Consideremos $\mathrm{N}$ vetores aleatórios $\mathrm{Y}_{1}, \ldots, \mathrm{Y}_{\mathrm{N}}$ de dimensões $M_{1}, \ldots, M_{N}$ respectivamente. $Y_{i j} e ́$, portanto, a j-ésima variável aleatória do vetor $\mathbf{Y}_{i}$ e $y_{i j}$ é a sua realização. Assim, a população é constituida de $N$ grupos (comumente chamados de clusters ou conglomerados), cada qual com $\mathrm{M}_{i}$ elementos, $i=1, \ldots, N$. No primeiro estágio, uma amostra de $n$ dos $N$ grupos é selecionada. No segundo, $m_{i}$ dos $M_{i}$ elementos do i-ésimo grupo (caso este tenha sido selecionado no primeiro estágio) são selecionados e constituem a amostra final.

Scott e Smith (1969) trabalham com amostragem em dois estágios onde $Y_{i 1}, \ldots, Y_{i M_{i}}, i=1, \ldots, N$, dados $\mu_{i}$ e $\sigma_{i}^{2}$, são independentes e têm distribuição normal commédia $\mu_{i}$ e variância $\sigma_{i}^{2}$. Assumem ainda distribuições a priori para os parâmetros acima. Neste capítulo, desenvolvemos uma teoria Bayesiana em amostragem em dois estágios, onde a distribuição que relaciona os elementos da população é o modelo geral descrito na Seção 1.1 e as distribuições a priori são as estabelecidas naquela mesma seção. A seguir serão feitas aplicações aos modelos binomial, poisson e normal.

\section{2. o modelo geral}

Consideremos uma população finita dividida em $\mathrm{N}$ grupos. O i-ésimo grupo possui $\mathrm{M}_{i}$ elementos com valores 
$\mathrm{Y}_{i 1}, \ldots, \mathrm{Y}_{i \mathrm{M}_{i}}\left(\mathrm{Y}_{\mathrm{i}}\right)$. No primeiro estágio uma amostra de $\mathrm{n}$ grupos é selecionada $(n \leqq N)$. No segundo estágio são seleciona das amostras de tamanhos $m_{i}\left(m_{i} \leqq M_{i}\right)$ de cada um dos grupos selecionados no primeiro estágio.

Fazemos as seguintes suposições acerca do modelo para os elementos da população:

i. Os $M_{i}$ elementos do i-ésimo grupo, dados $\alpha$ e $\phi$, são obser vações independentes tais que a função de densidade lou de probabilidade) de $Y_{i j}$ é dada em (1.2), isto é,

$$
\begin{aligned}
& \pi\left(y_{i j} / \alpha_{i}, \phi_{i}\right) \propto \exp \left\{\phi_{i}\left[y_{i j} \alpha_{i}-a\left(\alpha_{i}\right)\right]\right\} \\
& i=1, \ldots, N, j=1, \ldots, m_{i}, \\
& \text { com } \phi_{i}>0, \text { conhecido; }
\end{aligned}
$$
ii. $\quad-\alpha_{i} / \mu, \sigma^{2} \sim N\left(\mu, \sigma^{2}\right), i=1, \ldots, N$, independentes e $\pi(\mu) \propto$ constante, $\sigma^{2}$ conhecida.

Na realidade as suposições acima são exatamente aque las que foram feitas no Capítulo 1, onde trabalhamos com popu lações supostamente infinitas. A suposiçãoii nada mais é do que a estrutura a priori em dois estágios tomada anteriormente. Em primeiro lugar, suporemos $\sigma^{2}$ conhecida e, a seguir, as sumiremos uma distribuição a priori também parz $\sigma^{2}$. Deve-se notar que estas suposições são equivalentes a especificar uma superpopulação da qual uma população finita foi retirada. 
Para facilitar a notação, denotamos por s o conjun to dos índices $i$ dos grupos selecionados no primeiro estágio e por $s_{i}$ (ies), o conjunto dos índices $j$ dos elementos selecionados no segundo estágio.

Nosso interesse é fazer previsões para quantidades

do tipo

$$
T=\sum_{i=1}^{N} \sum_{j=1}^{M_{i}} p_{i j} Y_{i j}
$$

ou seja, uma combinação linear dos valores dos elementos da população. Por exemplo, tomando $p_{i j}=1$ e $p_{i j}=1 / \sum_{i=1}^{N} M_{i} t e-$ mos, respectivamente, o total e a média populacionais. Do ponto de vista Bayesiano, assumindo perda quadrática, estas quantidades devem ser previstas por

$$
\begin{aligned}
E\left[T / Y_{(s)}\right] & =E\left[\sum_{i=1}^{N} \sum_{j=1}^{M_{i}} p_{i j} Y_{i j} / Y_{(s)}\right]= \\
& =\sum_{i=1}^{N} \sum_{j=1}^{M_{i}} p_{i j} E\left[Y_{i j} / Y_{(s)}\right]
\end{aligned}
$$

onde $\mathbf{y}_{(\mathrm{s})}$ denota a amostra observada, isto é, a previsão é feita com base na média a posteriori de $\mathrm{T}$ dada a amostra ob servada. Evidentemente, se $y_{i j}$ pertence à amostra, a média a posteriori de $y_{i j}$ é $y_{i j}$. Nosso objetivo, portanto, é encontrar a média a posteriori de $Y_{i j}$ (ou aproximações, quando não for lossivel encontrar o valor exatol para elementos fora da amostra.

o cálculo da média a posteriori de $Y_{i j}$ é facilitado pelo seguinte resultado: 


$$
\begin{aligned}
& E\left[Y_{i j} / Y_{(s)}, \phi, \sigma^{2}\right]=E\left[E\left[Y_{i j} / \alpha, Y_{(s)}, \phi, \sigma^{2}\right] / Y_{(s)}, \phi, \sigma^{2}\right]=
\end{aligned}
$$

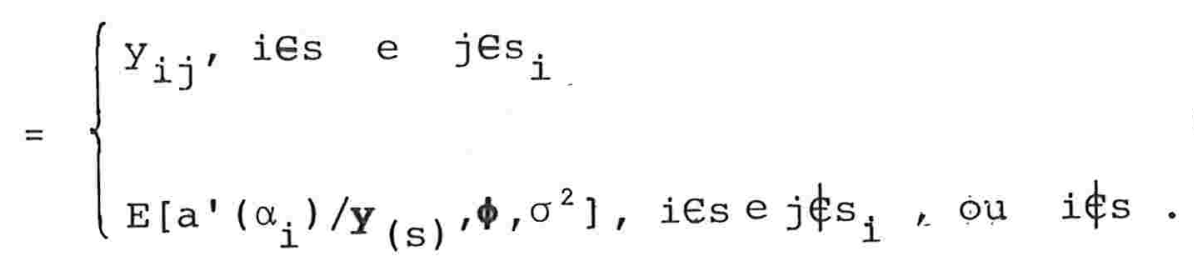

Logo, será necessário encontrar a distribuição a posteriori de a e calcular a média de $a^{\prime}\left(\alpha_{i}\right)$ nesta distribui ção. Na realidade, este cálculo, em geral, não pode ser feí to de forma exata. Uma solução é aproximar $E\left[a^{\prime}\left(\alpha_{i}\right) / y(s), \phi, \sigma^{2}\right]$ por $a^{\prime}\left(\tilde{\alpha}_{i}\right)$ onde $\tilde{\alpha}_{i}$ é a moda a posteriori de $\alpha_{i}$ (exatamente como foi feito nos capítulos anteriores). Outra solução é calcular a média a posteriori aproximada de $a^{\prime}\left(\alpha_{i}\right)$ baseado no método de Laplace (Seção 1.3).

O cālculo da covariância a posteriori de $\mathrm{Y}_{i j}$ e $\mathrm{Y}_{\ell q}$ ' necessário para calcular a variância a posteriori de T, é facilitado pelo resultado

$$
\begin{aligned}
& \operatorname{Cov}\left[Y_{i j}, Y_{\ell q} / y(s), \phi, \sigma^{2}\right]=E\left[\operatorname{Cov}\left[Y_{i j}, Y_{\ell q} / \alpha, y(s), \phi, \sigma^{2}\right] / y(s), \phi, \sigma^{2}\right]+
\end{aligned}
$$

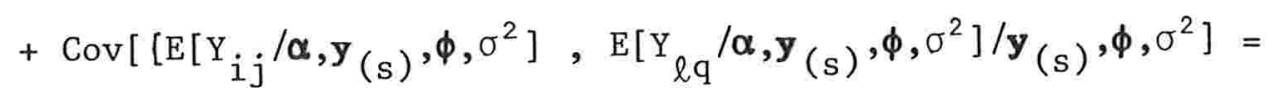

$$
\begin{aligned}
& \int \operatorname{Cov}\left[a^{\prime}\left(\alpha_{i}\right), a^{\prime}\left(\alpha_{\ell}\right) / y(s), \phi, \sigma^{2}\right], \\
& (i, j) \neq(l, q) \text { e }(i, j),(l, q) \notin s(*) \\
& =\left\{\mathrm{E}\left[\frac{\mathrm{a}^{\prime \prime}\left(\alpha_{i}\right)}{\phi_{i}} / y_{(s)}, \phi, \sigma^{<}\right]+\operatorname{Var}\left[\mathrm{a}^{\prime}\left(\alpha_{i}\right) / \mathbf{y}(s), \phi, \sigma^{2}\right],\right. \\
& (i, j)=(l, q) \text { e }(i, j),(l, q) \notin s \\
& 0,(i, j) \in S \quad \text { ou } \quad(l, q) \text { e } S
\end{aligned}
$$

(*) S denota o conjunto $\left\{(i, j): i \in s, j \in s_{i}\right\}$. 
Logo, o cálculo da covariância acima é unicamente baseado na distribuição a posteriori de $\boldsymbol{\alpha}$. Nas seções seguintes passaremos a encontrar esta distribuição e a propor aproximações para as médias e covariâncias a posteriori.

\subsubsection{Variância da distribuição a priori conhecida}

Sob as suposições feitas na seção 3.2 (com $\sigma^{2}$ conhe cida), a função de densidade a posteriori de $\alpha$ e $\mu$ é dada por

$$
\begin{aligned}
\pi\left(\alpha, \mu / y_{(s)}, \phi, \sigma^{2}\right) & \propto \exp \left\{\sum_{i \in s} \phi_{i}\left[y_{i} \alpha_{i}-m_{i} a\left(\alpha_{i}\right)\right]-\right. \\
& \left.-\frac{1}{2 \sigma^{2}} \sum_{i=1}^{N}\left(\alpha_{i}-\mu\right)^{2}\right\}
\end{aligned}
$$

onde $y_{i}=\sum_{j \in s_{i}} y_{i j}$, ies.

Integrando (3.4) em relação a $\mu$ temos a função de densidade a posteriori marginal de $\boldsymbol{\alpha}$,

$$
\begin{aligned}
\pi\left(\alpha / y(s), \phi, \sigma^{2}\right) & \propto \exp \left\{\sum_{i \in s} \phi_{i}\left[y_{i} \alpha_{i}-m_{i} a\left(\alpha_{i}\right)\right]-\right. \\
& \left.-\frac{1}{2 \sigma^{2}} \sum_{i=1}^{N}\left(\alpha_{i}-\alpha_{\bullet}\right)^{2}\right\}
\end{aligned}
$$

onde $\alpha .=\sum_{i=1}^{N} \frac{\alpha_{i}}{N}$.

Como já foi visto na seção anter:or é necessário encontrar a média a posteriori de $a^{\prime}\left(\alpha_{i}\right), i=1, \ldots, N$. Como esta não pode ser calculada de forma exata, encontraremos a moda $\tilde{\alpha}^{T}=\left(\tilde{\alpha}_{1}, \ldots, \tilde{\alpha}_{N}\right)$ de $(3.5)$ e utilizaremos a moda a poste 
riori de a' $\left(\alpha_{i}\right)$, isto é, a' $\left(\tilde{\alpha}_{i}\right)$, em lugar de sua média a pos teriori. Na Seção 3.3 será proposta uma outra aproximação baseada no método de Laplace, tal como foi descrito na seção 1. 3 .

As modas de (3.5) são obtidas pela solução do sistema de equações:

$$
\left\{\begin{array}{l}
a^{\prime}\left(\tilde{\alpha}_{i}\right)=\frac{y_{i}}{m_{i}}-\frac{\tilde{\alpha}_{i}-\tilde{\alpha}}{m_{i} \phi_{i} \sigma^{2}}, \text { ies } \\
\tilde{\alpha}_{i}=\tilde{\alpha}, \text { i申s }
\end{array}\right.
$$

onde $\tilde{\alpha}_{.}=\sum_{i=1}^{N} \frac{\tilde{\alpha}_{i}}{N}$.

E claro que dependendo da função $a^{\prime}(\cdot)$ a solução acima só pode ser encontrada por métodos iterativos. o método de Newton Raphson em (1.9) pode ser usado, bastando tomar

$$
C_{i(k)}= \begin{cases}\phi_{i} y_{i}-m_{i} \phi_{i} a^{\prime}\left(\alpha_{i(k)}\right)-\frac{\alpha_{i(k)}-\alpha \cdot(k)}{\sigma^{2}}, & \text { ies } \\ -\frac{\alpha_{i(k)}-\alpha \cdot(k)}{\sigma^{2}}, \text { iфs } & \end{cases}
$$

e a matriz $\mathbf{C}_{(\mathrm{k})}^{-1}=\mathbf{D}_{(\mathrm{k})}$ com elementos dados por

$$
D_{i j(k)}=u_{i(k)} \delta_{i j}+\frac{u_{i(k)} u_{j(k)}}{N\left(\sigma^{2}-u_{\cdot(k)}\right)}, i, j=1, \ldots, N,
$$

unde

$$
u_{i(k)}=\left\{\begin{array}{l}
\left(m_{i} \phi_{i} a^{n}\left(\alpha_{i(k)}\right)+\frac{1}{\sigma^{2}}\right)^{-1}, \text { ies } \\
\sigma^{2}, i \notin s
\end{array}\right.
$$


$u_{\cdot(k)}=\sum_{i=1}^{N} \frac{u_{i(k)}}{N}$

Assim, encontrado o vetor $\tilde{\boldsymbol{a}}$, um previsor Bayesiano aproximado de $T=\sum_{i=1}^{N} \sum_{j=1}^{M_{i}} p_{i j} Y_{i j} \quad$ é

$$
\begin{aligned}
\tilde{T} & =\sum_{i \in s} \sum_{j \in s_{i}} p_{i j} Y_{i j}+\sum_{i \in s} \sum_{j \notin s} p_{i j} a^{\prime}\left(\tilde{\alpha}_{i}\right)+ \\
& +\sum_{i \notin s} \sum_{j=1}^{M_{i}} p_{i j} a^{\prime}\left(\tilde{\alpha}_{0}\right) \ldots
\end{aligned}
$$

$\tilde{\mathrm{T}}$ é, portanto, a soma de três parcelas: a primeira correspon dente aos elementos da amostra, a segunda, aos elementos fora da amostra porém pertencentes aos grupos selecionados e a ter ceira, aos elementos dos grupos não selecionados.

É possivel fazer uma aproximação normal para a função de verossimilhança (ver Seção 1.2.1). Neste caso, encontra-se a média a posteriori aproximada $(\tilde{\alpha} *)$ de $\alpha$ sem necessidade de um processo iterativo. Nãoé difícil mostrar que a distribuição a posteriori aproximada é normal multivariada e que $\tilde{\alpha}_{i}^{*}$ é dado por

$$
\tilde{\alpha}_{i}^{*}=\left\{\begin{array}{l}
\frac{\hat{\alpha}_{i}}{\frac{v_{i}}{v_{i}}+\frac{\hat{\alpha}}{\sigma^{2}}}, \text { ies } \\
\frac{1}{v_{i}}+\frac{1}{\sigma^{2}} \\
\hat{\alpha}_{0}, \text { i申s }
\end{array}\right.
$$

onde $\quad \hat{\alpha}_{i}=a^{\prime-1}\left(\frac{y_{i}}{m_{i}}\right)$, ies, $v_{i}=\left[m_{i} \phi_{i} a^{\prime \prime}\left(\hat{\alpha}_{i}\right)\right]^{-1}$, ies, e 


$$
\hat{\alpha}_{0}=\frac{\sum_{i \in S} \frac{\hat{\alpha}_{i}}{\sigma^{2}+v_{i}}}{\sum_{i \in S} \frac{1}{\sigma^{2}+v_{i}}} .
$$

Logo, um previsor Bayesiano aproximado de T é dado por (3.9) com $a^{\prime}\left(\tilde{\alpha}_{i}\right)$ e $a^{\prime}\left(\tilde{\alpha}_{\bullet}\right)$ substituidos, respectivamente, por $a^{\prime}\left(\tilde{\alpha}_{i}^{*}\right)$ e $a^{\prime}\left(\hat{\alpha}_{0}\right)$.

\subsubsection{Variância da distribuição a priori desconhecida}

\section{Suponhamos agora que $\sigma^{2}$ seja desconhecida e que} uma distribuição a priori $x^{2}$ invertida seja adotada como nos capítulos anteriores. Neste caso, a função de densidade a posteriori de $\boldsymbol{\alpha}, \mu$ e $\sigma^{2}$ é

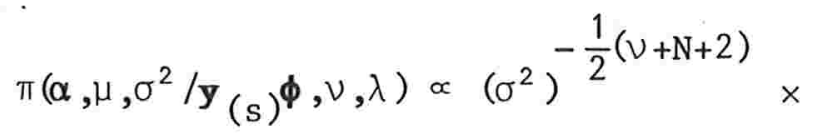

$$
\begin{aligned}
& \times \exp \left\{\sum_{i \in s} \phi_{i}\left[y_{i} \alpha_{i}-m_{i} a\left(\alpha_{i}\right)\right]-\frac{1}{2 \sigma^{2}}\left[\nu \lambda+\sum_{i=1}^{N}\left(\alpha_{i}-\mu\right)^{2}\right]\right\}
\end{aligned}
$$

Integrando (3.10) com respeito a $\mu$ e $\sigma^{2}$ obtem-se a seguinte função de densidade a posteriori marginal de $\boldsymbol{\alpha}$ :

$$
\begin{aligned}
& \pi(\alpha / y(s), \phi, \nu, \lambda) \propto\left[\nu \lambda+\sum_{i=1}^{N}\left(\alpha_{i}-\alpha_{0}\right)^{2}\right]^{-\frac{1}{2}(\nu+N-1)} \times \\
& \times \exp \left\{\sum_{i \in s} \phi_{i}\left[y_{i} \alpha_{i}-m_{i} a\left(\alpha_{i}\right)\right]\right\}
\end{aligned}
$$

(*) Note-se que não é possível tomar $\nu=0$ pois, neste caso, esta função de densidade tenderia a $\infty$ quando $\alpha_{1}=\ldots=\alpha_{N}$. 
A moda a posteriori é obtida pela solução do sistema de equações em (3.6) com $\sigma^{2}$ substituida por

$$
\tilde{\sigma}^{2}=\frac{\nu \lambda+\sum_{i=1}^{N}\left(\tilde{\alpha}_{i}-\tilde{\alpha}_{\bullet}\right)^{2}}{\nu+N-1}
$$

e o previsor $\tilde{\mathrm{T}}$ de $\mathrm{T}$ tem a mesma forma de (3.9).

Para encontrar a solução do sistema de equações bas ta utilizar o método de Newton Raphson em (1.9) com

$$
c_{i(k)}= \begin{cases}\phi_{i} y_{i}-m_{i} \phi_{i} a^{\prime}\left(\alpha_{i(k)}\right)-\frac{\alpha_{i(k)}-\alpha}{\sigma_{(k)}^{2}}, & i \in s \\ -\frac{\alpha_{i(k)}-\alpha \cdot(k)}{\sigma_{(k)}^{2}}, \text { i申s } & (3.11)\end{cases}
$$

e

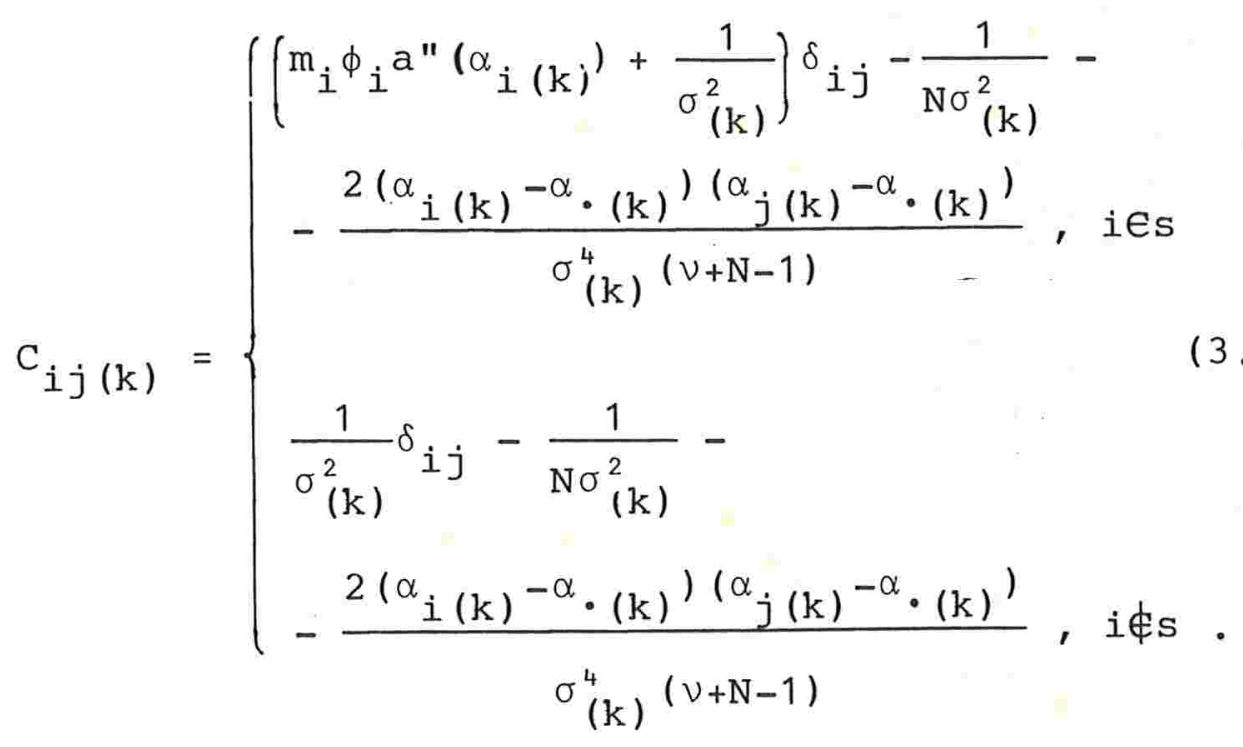

É possível fazer uma aproximação normal para a função de verossimilhança, porém esta rão é de grande utilidade pois, ao contrário do caso em que $\sigma^{2}$ é conhecida, não conduz a solução explícita para o estimador modal de $\alpha$. 
Na seção seguinte encontraremos a média a posteriori aproximada de $a^{\prime}\left(\alpha_{1}\right), \ldots, a^{\prime}\left(\alpha_{N}\right)$ baseados no método de Laplace para integrais. Assim, por (3.2), será possível encontrar apro ximações para a média a posteriori de $\mathbf{y}_{1}, \ldots, \mathbf{y}_{\mathrm{N}}$ e, consequentemente, para a média a posteriori de $\mathrm{T}$.

3.3. Cálculo dos momentos a posteriori aproximados pelo método de Laplace

o método de Laplace para integrais descrito na Seção 1.3 é facilmente aplicado ao cálculo dos momentos aproximados de $a^{\prime}\left(\alpha_{1}\right), \ldots, a^{\prime}\left(\alpha_{N}\right)$. Por esta razão omitiremos detalhes sobre a obtenção dos resultados.

3.3.1. Variância da distribuição a priori conhecida

Quando a variância da distribuição a priori é conhe cida, a média a posteriori aproximada de a' $\left(\alpha_{\ell}\right)$, obtida pelo método de Laplace, é

$$
\begin{aligned}
& \hat{\mathrm{E}}\left[\mathrm{a}^{\prime}\left(\alpha_{\ell}\right) / \mathbf{Y}(\mathrm{s}), \phi, \sigma^{2}\right]=\left(\frac{\operatorname{det} \mathrm{D}^{*}}{\operatorname{det} \mathrm{D}}\right)^{\frac{1}{2}} \mathrm{a}^{\prime}\left(\alpha_{\ell}^{*}\right) \times \\
& \times \exp \left\{\sum_{i=1}^{N}-\frac{1}{2 \sigma^{2}}\left[\left(\alpha_{i}^{*}-\alpha_{\cdot}^{*}\right)^{2}-\left(\tilde{\alpha}_{i}-\tilde{\alpha}_{\bullet}\right)^{2}\right]+\right. \\
& \left.+\sum_{i \operatorname{es}_{s}}\left[\phi_{i} Y_{i}\left(\alpha_{i}^{*}-\tilde{\alpha}_{i}\right)-m_{i} \phi_{i}\left(a\left(\alpha_{i}^{*}\right)-a\left(\tilde{\alpha}_{i}\right)\right)\right]\right\}, \ell=1, \ldots, N,
\end{aligned}
$$


onde $\tilde{\alpha}_{i}, i=1, \ldots, N$, é obtido pelo método de Newton Raphson em (1.9) com $c_{i(k)}$ e $c_{(k)}^{-1}=D_{(k)}$ dados em (3.7) e (3.8) res pectivamente; $\alpha_{i}^{*}, i=1, \ldots, N$, também é obtido pelo mesmo método com

$$
c_{i(k)}=\left\{\begin{array}{l}
\phi_{i} y_{i}-m_{i} \phi_{i} a^{\prime}\left(\alpha_{i(k)}\right)- \\
-\frac{\alpha_{i(k)}-\alpha \cdot(k)}{\sigma^{2}}+\frac{\delta_{i \ell} a^{\prime \prime}\left(\alpha_{i(k)}\right)}{a^{\prime}\left(\alpha_{i(k)}\right)}, i \in s \\
-\frac{\alpha_{i(k)}-\alpha \cdot(k)}{\sigma^{2}}+\frac{\delta_{i l^{2}} a^{\prime}\left(\alpha_{i(k)}\right)}{a^{\prime}\left(\alpha_{i(k)}\right)}, i \notin s .
\end{array}\right.
$$

e com os elementos de $\mathbf{C}_{(\mathrm{k})}^{-1}=\mathbf{D}_{(\mathrm{k})}^{*}$ dados por

$$
\begin{aligned}
& D_{i j(k)}^{*}=w_{i(k)} \delta_{i j}+\frac{w_{i(k)} w_{j(k)}}{N\left(\sigma^{2}-w_{(k)}\right)}, i, j=1, \ldots, N, \\
& w_{i(k)}=\left\{\begin{array}{l}
-\left(\delta_{i \ell} a_{i(k)}+m_{i} \phi_{i} a^{n}\left(\alpha_{i(k)}\right)+\frac{1}{\sigma^{2}}\right)^{-1}, i \in s \\
\left(\delta_{i \ell} a_{i(k)}+\frac{1}{\sigma^{2}}\right)^{-1}, \text { i申s, }
\end{array}\right.
\end{aligned}
$$

com $a_{i(k)}$ dado por (1.27) e onde $\mathbf{D}$ e $\mathbf{D} *$ são, respectivamente, iguais às matrizes $\mathbf{D}_{(\mathrm{k})}$ e $\mathbf{D}_{(\mathrm{k})}^{*}$ obtidas na última iteração das aplicações do método de Newton Raphson.

Agora, por (3.1) e (3.2), vemos que é razoável apro ximar a média a posteriori de $\mathrm{T}$ por

$$
\hat{T}=\sum_{i \varrho s s} \sum_{j \in s_{i}} p_{i j} Y_{i j}+\sum_{i=1}^{N} \sum_{j \phi_{s}} p_{i j} \hat{E}\left[a^{\prime}\left(\alpha_{i}\right) / y(s), \phi, \sigma^{2}\right] .
$$


Logo, podemos tomar $\hat{\mathrm{T}}$ como um previsor Bayesiano aproximado de T.

É possível encontrar também a variância a posteriori aproximada de $\mathrm{T}$ da seguinte forma:

$$
\begin{aligned}
& \hat{\operatorname{Var}}\left[\mathrm{T} / \mathrm{Y}_{(\mathrm{s})}, \phi, \sigma^{2}\right]=\hat{\operatorname{Var}}\left[\sum_{i=1}^{\mathrm{N}} \sum_{j=1}^{\mathrm{M}} \mathrm{p}_{i j} \mathrm{Y}_{i j} / \mathrm{Y}_{(\mathrm{s})}, \phi, \sigma^{2}\right]= \\
& =\sum_{i=1}^{N} \sum_{j=1}^{M_{i}} \sum_{\ell=1}^{N} \sum_{j=1}^{M_{i}} p_{i j} P_{\ell q} \hat{\operatorname{Cov}}\left[Y_{i j}, Y_{\ell q} / Y_{(s)}, \phi, \sigma^{2}\right] .
\end{aligned}
$$

De (3.3) segue que para calcular as covariâncias acima basta encontrar a média e a covariância a posteriori aproximadas, respectivamente, de a" $\left(\alpha_{\ell}\right), \quad \ell=1, \ldots, N$ e do par a' $\left(\alpha_{\ell}\right)$ e a $\left(\alpha_{q}\right)$, $\ell, q=1, \ldots, N$

3.3.2. Variância da distribuição a priori desconhecida

Quando $\sigma^{2}$ é desconhecida, a média a posteriori aprọ ximada de $a^{\prime}\left(\alpha_{\ell}\right), \quad \ell=1, \ldots, N$, é dada por

$$
\begin{aligned}
& \hat{\mathrm{E}}\left[a^{\prime}\left(\alpha_{\ell}\right) / y(s), \phi, \nu, \lambda\right]=\left(\frac{\operatorname{det} \mathrm{D}^{*}}{\operatorname{det} \mathrm{D}}\right)^{\frac{1}{2}} \mathrm{a}^{\prime}\left(\alpha_{\ell}^{*}\right) \times \\
\times & \exp \left\{-\frac{1}{2}(\nu+N-1)\left[\log \left[\nu \lambda+\sum_{i=1}^{N}\left(\alpha_{i}^{*}-\alpha^{*}\right)^{2}\right]-\log \left[\nu \lambda+\sum_{i=1}^{N}\left(\tilde{\alpha}_{i}-\tilde{\alpha}_{\bullet}\right)^{2}\right]\right]+\right. \\
& \left.+\sum_{i \in{ }^{\prime}}\left[\phi_{i} Y_{i}\left(\alpha_{i}^{*}-\tilde{\alpha}_{i}\right)-m_{i} \phi_{i}\left(a\left(\alpha_{i}^{*}\right)-a\left(\tilde{\alpha}_{i}\right)\right)\right]\right\}, \ell=1, \ldots, N,
\end{aligned}
$$

onde $\tilde{\alpha}_{i}, i=1, \ldots, N$, é obtido pelo método de Newton Raphson em (1.9) $\mathrm{com}_{i(k)}$ e $C_{i j(k)}$ dados por (3.11) e $(3.12) ; \alpha_{i}^{*}, i=1, \ldots, N$, 
também é obtido pelo mesmo método com

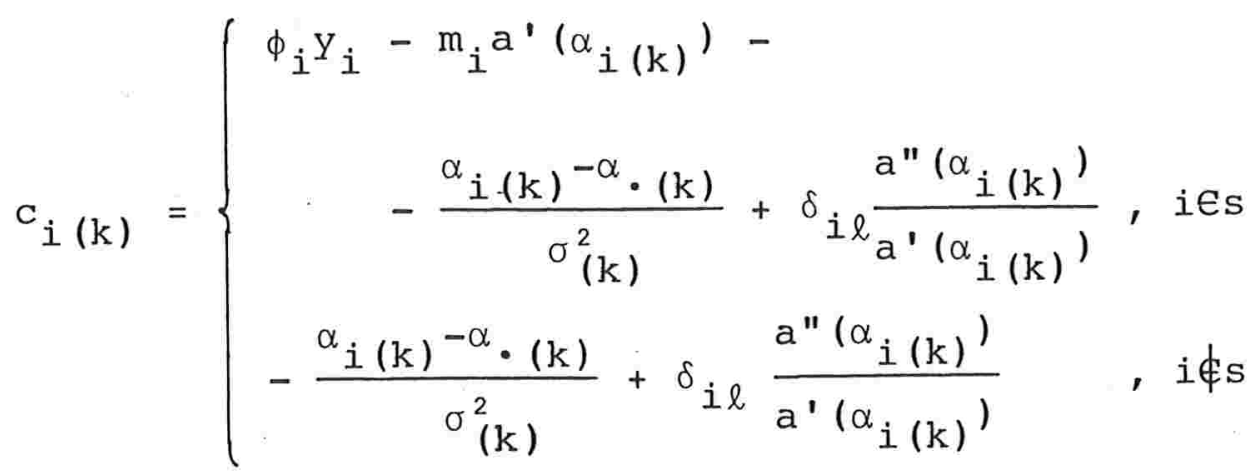

e os elementos de $\mathbf{C}_{(\mathrm{k})}$ dados por

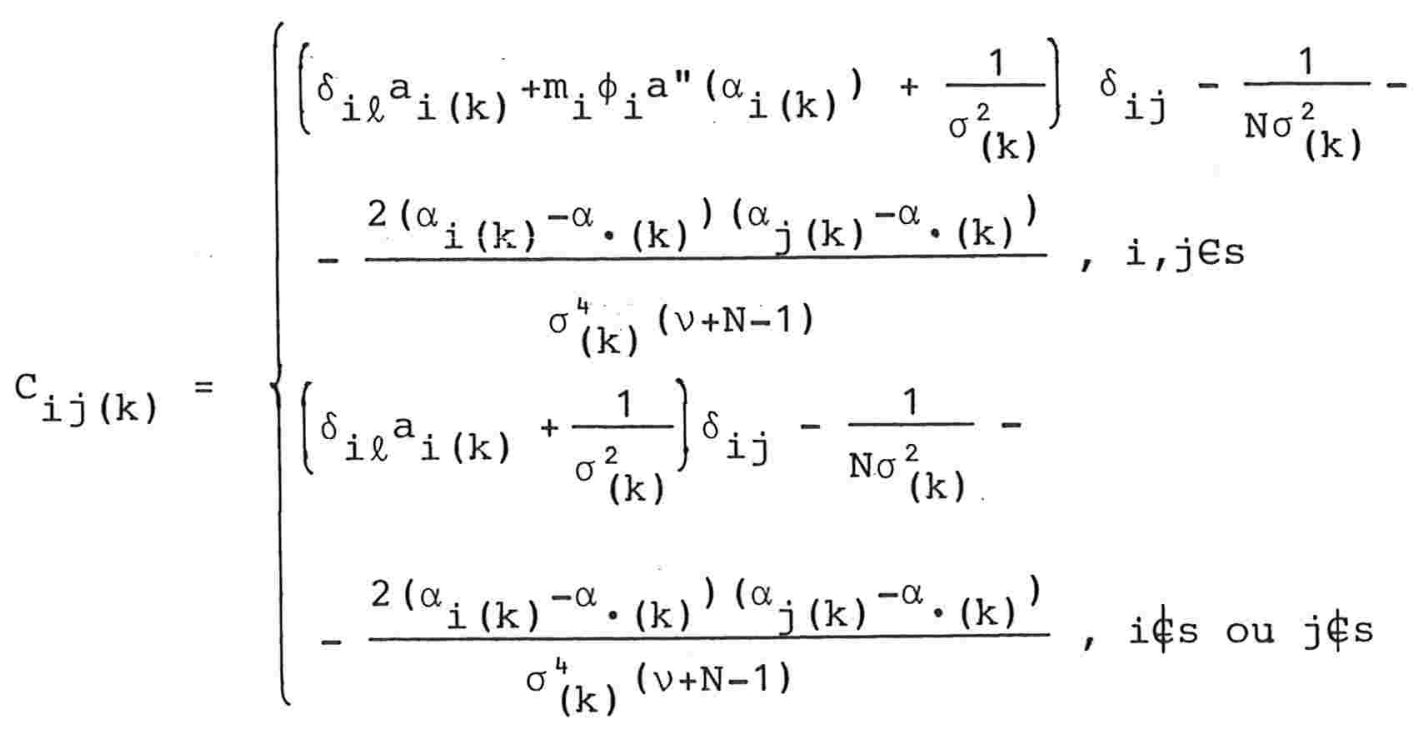

$\operatorname{com} \quad \sigma_{(k)}^{2}=\frac{\nu \lambda+\sum_{i=1}^{N}\left(\alpha_{i(k)}-\alpha \cdot(k)\right)^{2}}{\nu+N-1}$, e onde $a_{i(k)}$ é ob-

tido por (1.27) e D e D* são, respectivamente, iguais às matrí zes inversas calculadas na última iteração das aplicações do método de Newton Raphson para a obtenção de $\tilde{\alpha}$ e $\alpha$ *

Logo, da mesma fornıd que na seção anterior, vemos que é razoável tomar

$$
\hat{T}=\sum_{i \in s} \sum_{j \in s_{i}} p_{i j} Y_{i j}+\sum_{i=1}^{N} \sum_{j \notin s_{i}} p_{i j} \hat{E}\left[a \alpha^{\prime}\left(\alpha_{i}\right) / y(s), \phi, \nu, \lambda\right]
$$


como um previsor Bayesiano aproximado de $\mathrm{T}$ e que é possível encontrar pelo método de Laplace uma aproximação para a sua variância a posteriori.

\subsection{Alguns modelos especiais}

3.4.1. O modelo binomial

O processo de amostragem em dois estágios descrito na Seção $3.2 \operatorname{com} \alpha_{i}=\log \left[\Theta_{i} /\left(1-\theta_{i}\right)\right], 0<\theta_{i}<1, \phi_{i}=1$ e $a\left(\alpha_{i}\right)=\log \left(1+e^{\alpha}\right), \quad i=1, \ldots, N$, é tal que

i. os $M_{i}$ elementos do i-ésimo grupo, dados $M_{i}$ e $\theta_{i}$, são observações independentes de tal forma que

$$
\begin{aligned}
& \mathrm{Y}_{i} / \mathrm{M}_{i}, \Theta_{i} \sim \mathrm{B}\left(\mathrm{M}_{i}, \Theta_{i}\right), i=1, \ldots, \mathrm{N}, \\
& \text { onde } \mathrm{Y}_{i}=\sum_{j=1}^{\mathrm{M}_{i}} \mathrm{Y}_{i j} ;
\end{aligned}
$$

ii. $\quad \log \frac{\theta_{i}}{1-\theta_{i}} / \mu, \sigma^{2} \sim N\left(\mu, \sigma^{2}\right), i=1, \ldots, N$, independentes,

$$
\pi(\mu) \propto \text { constante }
$$

e,para $\sigma^{2}$ desconhecida, $\frac{\nu \lambda}{\sigma^{2}} / \nu, \lambda \sim x_{\nu}^{2}, \nu>0, \lambda>0$ e, dados $\nu$ e $\lambda, \mu$ e $\sigma^{2}$ são independentes.

Todos os resultados obtidos nas seções anteriores, tanto para $\sigma^{2}$ conhecida como desconhecida, podem ser facilmente obtidos na situação acima, isto é, no modelo binomial. 
Estes resultados serão omitidos aqui pois para obtê-los basta lembrar que a $\left(\alpha_{i}\right)=\log \left(1+e^{\alpha^{i}}\right)$ e $\phi_{i}=1, i=1, \ldots, N$, e ut $\underline{i}$ lizar os resultados das seções anteriores.

3.4.2. O modelo de Poisson

Consideremos o processo de amostragem em dois estágios descrito na seção 3.2 , porém $\operatorname{com} \alpha_{i}=\log \theta_{i}, \theta_{i}>0, \phi_{i}=1$ e $a\left(\alpha_{i}\right)=e^{\alpha_{i}}, i=1, \ldots, N$, de forma que

i. os $M_{i}$ elementos do i-ésimo grupo, dado $\Theta_{i}$, são observações independentes e que

$$
Y_{i j} / \Theta_{i} \sim P\left(\Theta_{i}\right), i=1, \ldots, N, j=1, \ldots, M_{i} ;
$$

ii. $\quad \log _{i} / \mu, \sigma^{2} \sim N\left(\mu, \sigma^{2}\right), i=1, \ldots, N$, independentes,

$$
\pi(\mu) \propto \text { constante }
$$

e, para $\sigma^{2}$ desconhecida, $\frac{\nu \lambda}{\sigma^{2}} / \nu, \lambda \sim x_{\nu}^{2}, \nu>0, \lambda>0$ e, dados $\nu$ e $\lambda, \dot{\mu}$ e $\sigma^{2}$ são independentes.

Nesta situação, para obter os resultados das seções anteriores basta lembrar que $a\left(\alpha_{i}\right)=e^{\alpha_{i}}$ e $\phi_{i}=1, i=1, \ldots, N$.

\subsubsection{O modelo normal}

o processo de amostragem em dois estágios descrito na Seção $3.2 \operatorname{com} \alpha_{i}=\theta_{i}, \phi_{i}=1 / \sigma_{i}^{2}$ e $a\left(\alpha_{i}\right)=\alpha_{i}^{2} / 2$ é tal que 
i. os $M_{i}$ elementos do i-ésimo grupo, dados $\theta_{i}$ e $\sigma_{i}^{2}$, são observações independentes de tal forma que

$$
Y_{i j} / \Theta_{i}, \sigma_{i}^{2} \sim N\left(\Theta_{i}, \sigma_{i}^{2}\right) \quad i=1, \ldots, N, j=1, \ldots, M_{i} ;
$$

ii.

$$
\theta_{i} / \mu, \sigma^{2} \sim N\left(\mu, \sigma^{2}\right) \quad i=1, \ldots, N \text {, independentes, }
$$$$
\pi(\mu) \propto \text { constante }
$$

e $\quad \sigma^{2}$ e $\sigma_{i}^{2}, i=1, \ldots, N$, são, a princípio, consideradas conhecidas. Este modelo é discutido em Scott e Smith (1969) .

\subsubsection{Variâncias conhecidas}

Supondo que $\sigma^{2}$ e $\sigma_{i}^{2}, i=1, \ldots, N$, são conhecidas, a distribuição a posteriori de $\theta$ (ver (3.5)) é normal multivariada. As médias a posteriori são facilmente obtidas através de (3.6) e são dadas por

$$
\tilde{\Theta}_{i}=d_{i} \bar{y}_{i}+\left(1-d_{i}\right) \tilde{\Theta}
$$

onde

$$
\begin{aligned}
& d_{i}=\left\{\begin{array}{l}
\frac{\sigma^{2}}{\sigma^{2}+\sigma_{i}^{2} / m_{i}}, \text { ies } \\
0, i \notin s
\end{array}\right. \\
& \tilde{\theta}_{\cdot}=\frac{\sum_{i \in s_{i} \bar{y}_{i}} \sum_{i \in s} d_{i}}{}
\end{aligned}
$$

e $\bar{y}_{i}=\sum_{j \in s_{i}} \frac{y_{i j}}{m_{i}} \cdot A$ matriz de covariância a posteriori $\mathbf{D}$ é 
obtida calculando-se a inversa da matriz de informação a pos teriori C. A matriz C tem elementos dados por

$$
c_{i j}=\frac{\delta_{i j}}{u_{i}}-\frac{1}{N \sigma^{2}}, \quad i, j=1, \ldots, N
$$

onde

$$
u_{i}=\left\{\begin{array}{l}
\left(\frac{m_{i}}{\sigma_{i}^{2}}+\frac{1}{\sigma^{2}}\right)^{-1}, i \in s \\
\sigma^{2}, i \notin s
\end{array}\right.
$$

$u_{\cdot}=\sum_{i=1}^{N} \frac{u_{i}}{N}$

Invertendo a matriz acima temos que $\mathbf{D}$, a matriz de covariância a posteriori, tem elementos

$$
D_{i j}=u_{i} \delta_{i j}+\frac{u_{i} u_{j}}{N\left(\sigma^{2}-u_{0}\right)}, \quad i, j=1, \ldots, N \text {. }
$$

Seja

$$
\xi^{2}=\left(\sum_{i \in s}\left(\sigma^{2}+\frac{\sigma_{i}^{2}}{m_{i}}\right)^{-1}\right)^{-1}
$$

Então, $D_{i j}$ pode ser escrita como

$$
D_{i j}=\left\{\begin{array}{l}
\left(1-d_{i}\right)^{2} \xi^{2}+\left(1-d_{i}\right) \sigma^{2}, i=j \\
\left(1-d_{i}\right)\left(1-d_{j}\right) \xi^{2}, i \neq j .
\end{array}\right.
$$

De posse dos resultac'os a posteriori acima, pode mos propor um previsor $\tilde{\mathrm{T}}$ de $\mathrm{T}=\sum_{i=1}^{N} \sum_{j=1}^{\mathrm{M}} \mathrm{p}_{i j} \mathrm{Y}_{i j}$. Recordando que o previsor Bayesiano de $\mathrm{T}$ é sua média a posteriori (ver (3.1) e (3.2)) dada por 


$$
\begin{aligned}
& E\left[T / Y(s), \sigma^{2}, \sigma_{1}^{2}, \ldots, \sigma_{N}^{2}\right]=\sum_{i=1}^{N} \sum_{j=1}^{M_{i}} p_{i j} E\left[Y_{i j} / \mathbf{Y}(s), \sigma^{2}, \sigma_{1}^{2}, \ldots, \sigma_{N}^{2}\right]= \\
& =\sum_{i \in s} \sum_{j \in s_{i}} p_{i j} y_{i j}+\sum_{i \in s} \sum_{j \in s_{i}} p_{i j} E\left[a^{\prime}\left(\alpha_{i}\right) / y(s), \sigma^{2}, \sigma_{1}^{2}, \ldots, \sigma_{N}^{2}\right]+ \\
& +\sum_{i \notin s} \sum_{j=1}^{M} p_{i j} E\left[a^{\prime}\left(\alpha_{i}\right) / Y(s), \sigma^{2}, \sigma_{1}^{2}, \ldots, \sigma_{N}^{2}\right]
\end{aligned}
$$

e que $a^{\prime}\left(\alpha_{i}\right)=\theta_{i}$, temos que

$$
\tilde{T}=\sum_{i \in S_{s}} \sum_{j \in s_{i}} p_{i j} Y_{i j}+\sum_{i \in s} \sum_{j \notin s} p_{i j} \tilde{\Theta}_{i}+\sum_{i \notin s} \sum_{j=1}^{M_{i}} p_{i j} \tilde{\Theta}_{\bullet} \cdot(3.15)
$$

A variância a posteriori de T é obtida utilizando-se (3.3) que, no modelo normal, se reduz a

$$
\operatorname{Cov}\left[Y_{i j}, Y_{\ell q} / Y_{(s)}, \sigma^{2}, \sigma_{1}^{2}, \ldots, \sigma_{N}^{2}\right]=\left\{\begin{array}{r}
\operatorname{Cov}\left[\Theta_{i}, \Theta_{\ell} / Y_{(s)}, \sigma^{2}, \sigma_{1}^{2}, \ldots, \sigma_{N}^{2}\right], \\
(i, j) \neq(l, q) e(i, j),(l, q) \notin s \\
\sigma_{i}^{2}+\operatorname{Var}\left[\Theta_{i} / \mathbf{Y}(s), \sigma^{2}, \sigma_{1}^{2}, \ldots, \sigma_{N}^{2}\right], \\
(i, j)=(l, q) \text { e }(i, j),(l, q) \notin s \\
0,(i, j) \in s \quad \text { ou }(l, q) \in s .
\end{array}\right.
$$

ou ainda

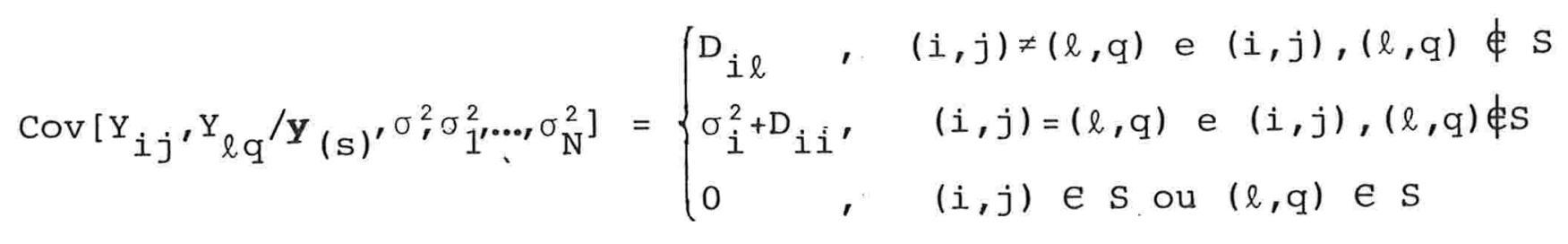
com $D_{i \ell}, i, \ell=1, \ldots, N$, dado em $(3.14)$. 
$\operatorname{Var}\left[\mathrm{T} / \mathrm{Y}(\mathrm{s}), \sigma^{2}, \sigma_{1}^{2}, \ldots, \sigma_{N}^{2}\right]=\operatorname{Var}\left[\sum_{i=1}^{N} \sum_{j=1}^{M_{i}} \mathrm{p}_{i j} \mathrm{Y}_{i j} / \mathrm{Y}(\mathrm{s}), \sigma^{2}, \sigma_{1}^{2}, \ldots, \sigma_{N}^{2}\right]$

$$
\begin{aligned}
& =\sum_{i=1}^{N} \sum_{j \in s_{i}} \underline{p}_{i j}^{2}\left(\sigma_{i}^{2}+D_{i i}\right)+ \\
& +\sum_{i=1}^{N} \sum_{j \notin s_{i}} \sum^{N}{ }^{*} \sum_{q \in s_{\ell}^{*}}^{N} p_{i j} p_{\ell q} D_{i \ell}
\end{aligned}
$$

onde a somatória dupla $\sum_{\ell=1}^{N} \sum_{\mathrm{q} \in \mathrm{s}_{\ell}}^{*}$ exclui as parcelas em que $(i, j)=(l, q)$.

Scott e Smith (1969) mostram que T̃ em (3.15) é o previsor que minimiza o erro quadrático médio na classe de tô dos os previsores lineares de T. Uma extensão deste resultado para o caso de mais de dois estágios é discutida em Malec e Sedransk (1985).

\subsubsection{Variâncias desconhecidas}

$\mathrm{Na}$ seção anterior supusemos $\sigma^{2}, \sigma_{1}^{2}, \ldots, \sigma_{\mathrm{N}}^{2}$ conhecidas. Eliminemos agora esta suposição e tomemos distribuições a priori $x^{2}$ invertidas para estes parâmetros como proposto na Seção 2.3 .2 .

Nesta situação, a função de densidade a posteriori

de $\theta$ é

$$
\begin{aligned}
& \pi(\boldsymbol{\theta} / \mathrm{y}(\mathrm{s}), \nu, \lambda) \propto\left[\nu \lambda+\sum_{i=1}^{N}\left(\Theta_{i}-\theta_{0}\right)^{2}\right]^{\frac{1}{2}(\nu+N-1)} \times \\
& \times \operatorname{T†}_{i \in S}\left[\nu_{i} \lambda_{i}+S_{i}^{2}+m_{i}\left(\bar{y}_{i}-\Theta_{i}\right)^{2}\right]^{\frac{1}{2}\left(\nu_{i}+m_{i}\right)}
\end{aligned}
$$

(*) - Pode-se notar que é possível tomar $\nu_{i}=0, i=1, \ldots, N$, porém $\nu$ deve ser positivo uma vez que se $\nu$ for igual a zero esta função de densidade tenderia a $\infty$ quando $\theta_{1}=\ldots=\theta_{\mathrm{N}}$. 
onde

$$
S_{i}^{2}=\sum_{j \in i}\left(y_{i j}-\bar{y}_{i}\right)^{2} \text {, ies. }
$$

A moda a posteriori de $\boldsymbol{\theta}$ é obtida pela solução do sistema de equações

$$
\tilde{\theta}_{i}=\tilde{d}_{i} \bar{y}_{i}+\left(1-\tilde{d}_{i}\right) \tilde{\theta} \text {. }
$$

onde

$$
\begin{aligned}
& \tilde{d}_{i}=\left\{\begin{array}{l}
\frac{\tilde{\sigma}^{2}}{\tilde{\sigma}^{2}+\tilde{\sigma}_{i} / m_{i}} \\
0, i \notin s
\end{array}\right. \\
& \sigma^{2}=\frac{\nu \lambda+\sum_{i=1}^{N}\left(\tilde{\Theta}_{i}-\tilde{\Theta}_{0}\right)^{2}}{\nu+N-1}, \sigma_{i}^{2}=\frac{\nu_{i} \lambda_{i}-S_{i}^{2}+m_{i}\left(\tilde{\theta}_{i}-\bar{y}_{i}\right)^{2}}{\nu_{i}+m_{i}} \text {, ies e } \\
& \tilde{\theta}_{.}=\frac{\sum_{i \in s} \tilde{\mathrm{d}}_{i} \tilde{y}_{i}}{\sum_{i \in s} \tilde{\mathrm{d}}_{i}} .
\end{aligned}
$$

Note-se que estes estimadores modais são semelhantes àqueles em que se supunha que as variâncias eram conheci das, porém com $\sigma^{2}$ e $\sigma_{i}^{2}$, ies, substituidas por $\tilde{\sigma}^{2}$ e $\tilde{\sigma}_{i}^{2}$, ies, respectivamente.

Propomos então que o previsor Bayesiano de $\mathrm{T}$, isto é, sua média a posteriori, seja aproximado por

$$
\tilde{T}=\sum_{i e s s} \sum_{j e_{s}} p_{i} j Y_{i j}+\sum_{i e s s} \sum_{j s_{i}} \tilde{\Theta}_{i}+\sum_{i 申 s} \sum_{j=1}^{M_{i}} \tilde{\Theta} .
$$


onde $\tilde{\Theta}_{i}$ e $\tilde{\Theta}$. são fornecidos em (3.16).

Para encontrar a solução de (3.16) pode-se utilizar o método de Newton Raphson em (1.9) com

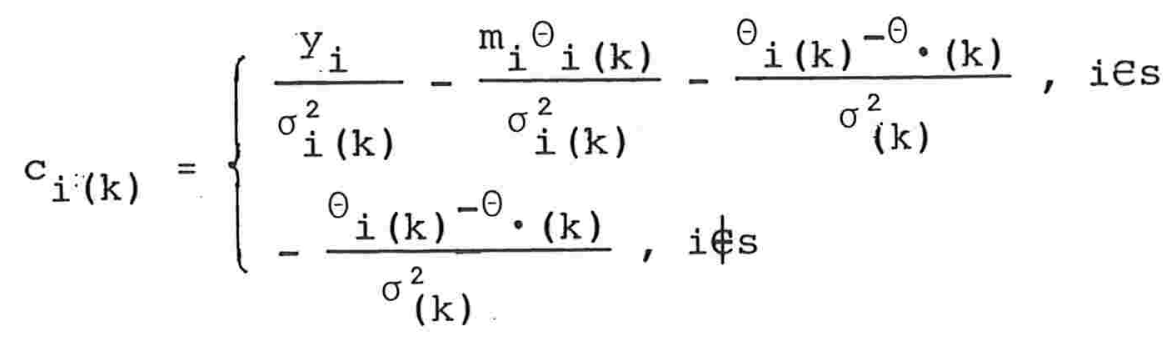

e

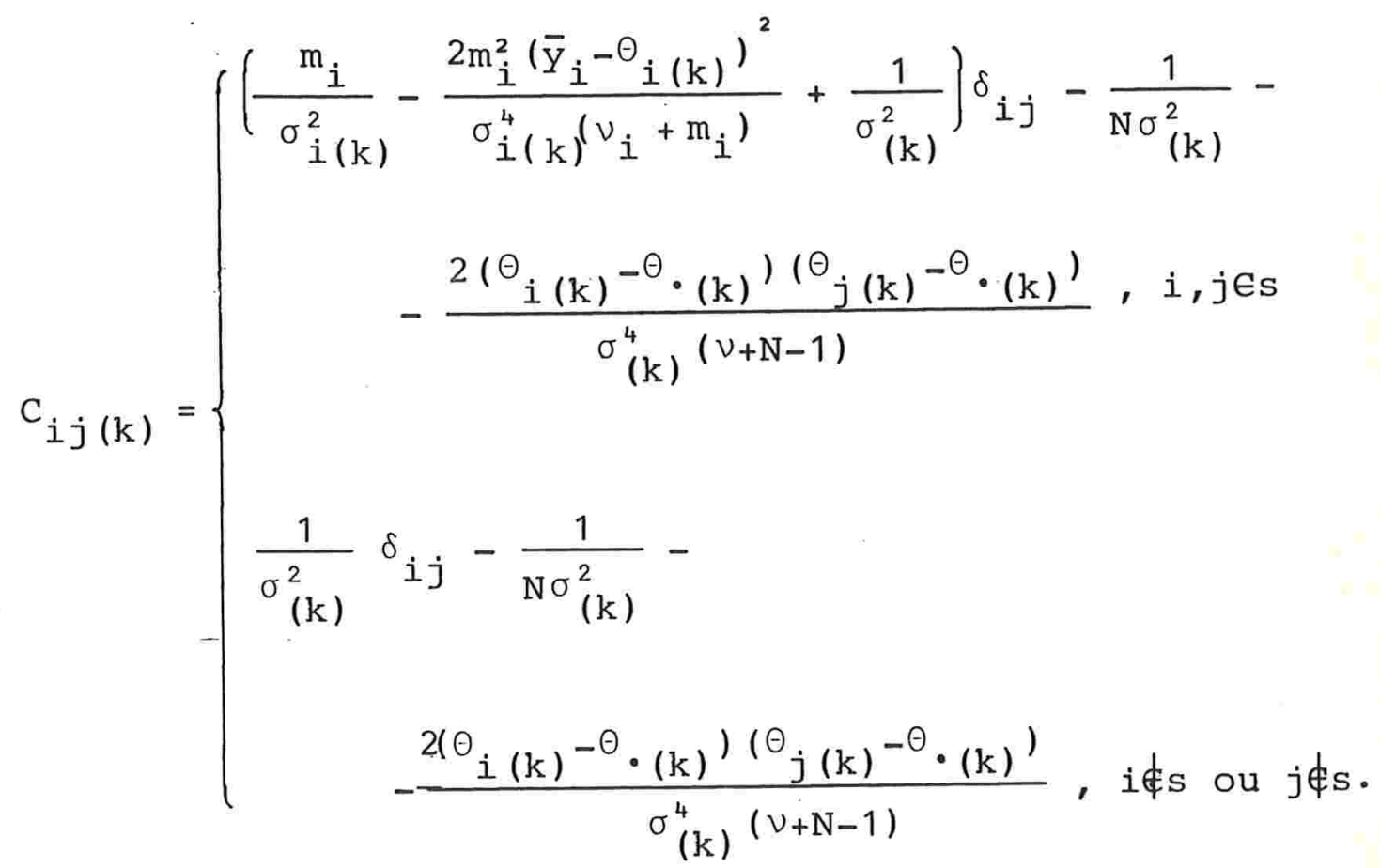

Seria interessante encontrar aproximações para as médias, covariāncias e variâncias a posteriori baseadas no método de Laplace para integrais. Porém, como já havia sido ressaltado anteriormente, este método não se aplica aqui pois $\Theta_{i}$ não é exclusivamente positivo.

Scott e Smith (1969) tratam a situação em que as variâncias são desconhecidas da seguinte forma: i) supõem que 
$\sigma_{1}^{2}=\ldots=\sigma_{\mathrm{N}}^{2}=\delta^{2}$ e que $\mathrm{m}_{i}=\mathrm{m}$, iEs ; iil atribuem a seguinte distribuição a priori para as variâncias

$$
p\left(d, \sigma^{2}, \delta^{2}\right) \propto \frac{d}{\sigma^{2} \delta^{2}}
$$

onde

$$
d=\frac{\sigma^{2}}{\sigma^{2}+\delta^{2} / m}
$$

Esta distribuição tem uma característica indesejável: depende do tamanho da amostra a ser selecionada. Os autores mostram que a média a posteriori de $Y_{i j}$ para elementos fora da amostra é

$$
E\left[Y_{i j} / Y_{(s)}\right]=\left\{\begin{array}{l}
\hat{a}_{\bar{y}_{i}}+(1-\hat{d}) \bar{y}, \text { ies } \\
\bar{y}, \text { iфs }
\end{array}\right.
$$

onde

$$
\begin{aligned}
& 1-\hat{\mathrm{d}}=\frac{n(m-1)}{r(n m-m-2)} \frac{I_{x}\left(\frac{n+1}{2}, \frac{n m-m-2}{2}\right)}{I_{x}\left(\frac{n-1}{2}, \frac{n m-m}{2}\right)}, \\
& r=\frac{n(m-1) x}{n-1}, \\
& x=\frac{\left.m \sum \bar{y}_{i}-\bar{y}\right)^{2}}{\sum_{i \in s} \sum_{j e s}\left(y_{i j}-\bar{y}\right)^{2}}, \\
& \bar{y}_{i}=\sum_{j \in s_{i}} \frac{y_{i j}}{m_{i}}, \\
& \bar{y}=\sum_{i \in s} \frac{\bar{y}_{i}}{n}
\end{aligned}
$$


e $I_{x}(p, q)$ denota a função beta incompleta. Os autores sugerem que seja utilizada a seguinte aproximação:

$$
\hat{\mathrm{d}} \cong \begin{cases}\frac{r-1}{r}, & r<1 \\ 0, & r \geqq 1\end{cases}
$$

uma vez que o cálculo exato de $\hat{d}$ é bastante trabalhoso. Este resultado também é citado em Cassel, Sarndal e Wretman (1977). 


\author{
CAPITULO 4 \\ APLICAÇÕES EM AMOSTRAS SIMULADAS
}

\title{
4.1. Introdução
}

Este capitulo tem como objetivo apresentar ilustra ções numéricas de resultados discutidos nos capítulos ante riores e fazer algumas comparações entre os diversos métodos propostos de estimação e previsão. Para tal, trabalha remos com populações e amostras simuladas. Como supusemos que $\alpha_{1}, \ldots, \alpha_{n}$ são independentes e têm distribuição a prio rio normal com média $\mu$ e variância $\sigma^{2}$, fixaremos valores para $\mu$ e $\sigma^{2}$ e geraremos, através de uma rotina de computador, $n$ observações, $\alpha_{1}, \ldots, \alpha_{n}$, independentes, segundo esta distribuição especificada. A seguir, como temos $\Theta_{i}=a^{\prime}\left(\alpha_{i}\right)$, calcularemos os $\Theta_{i}$ 's e geraremos $n$ amostras aleatórias de tamanhos $m_{1}, \ldots, m_{n}$ segundo uma distribuição de Bernoulli com média $\Theta_{i}$. Em seguida, repetiremos o procedimento para as distribuições de Poisson e normal, ambas com média $\Theta_{i}$. Nesta última também estabeleceremos valores para as variân cias $\sigma_{1}^{2}, \ldots, \sigma_{n}^{2}$. Em cada uma das três situações citadas, assumiremos a princípio que a variẩncia $\sigma^{2} e ́$ conhecida (na distribuição normal assumiremos ainda que $\sigma_{1}^{2}, \ldots, \sigma_{n}^{2}$ são também conhecidas) e encontraremos as estimativas pontuais pa ra $\theta_{1}, \ldots, \theta_{n}$ segundo os diversos métodos aproximados propostos nos capítulos anteriores. Numa segunda etapa, eliminaremos a suposição de conhecimento da(s) variância(s) e novamente estimaremos os parâmetros $\theta_{1}, \ldots, \theta_{n}$. 
Um procedimento bastante parecido com o descrito acima será utilizado para ilustrar a metodologia de previ são em amostragem em dois estágios e será descrito em deta1hes na Seção 4.5 .

\subsection{Estimação no modelo binomial}

Nesta seção ilustramos diversos resultados sobre estimação no modelo binomial apresentados na seção 2.1 . Es pecificamente, calculamos estimativas pontuais para os parâ metros de interesse $\theta_{1}, \ldots, \theta_{n}$ baseadas nos seguintes estima dores:

Estimador usual, isto é, a média amostral; Moda da distribuição à posteriori (dada em (2.1)), supondo $\sigma^{2}$ conhecida;

Média da distribuição a posteriori aproximada obtị da através de uma aproximação para a função de verossimiIhança (dada em (2.4)), supondo $\sigma^{2}$ conhecida;

4)

Média aproximada da distribuição a posteriori calculada pelo método de Laplace (Seção 2.1.3), supondo $\sigma^{2}$ co nhecida;

5)

Moda da distribuição a posteriori (Seção 2.1.2), suporido $\sigma^{2}$ desconhecida e

6)

Média aproximada da distribuição a posteriori calculada pelo método de Laplace (Seção 2.1.3) supondo $\sigma^{2}$ des- 
conhecida ${ }^{(*)}$.

Baseados na suposição a priori de que $\alpha_{1}, \ldots, \alpha_{n}$ são independentes e normalmente distribuidos com média $\mu$ e variância $\sigma^{2}$, fixamos $n=7, \mu=0$ e $\sigma^{2}=1$ e geramos 7 observações independentes segundo a distribuição $N(0,1)^{(* *)}$. Desta forma, de posse do vetor $\left(\alpha_{1}, \ldots, \alpha_{7}\right)$ calculamos $\theta_{i}=e^{\alpha} i /\left(1+e^{\alpha} i\right), i=1, \ldots, 7$. A seguir, geramos 7 amostras aleatórias (de tamanhos $m_{i}$ pré-fixados) segundo uma distri buição de Bernoulli com média $\theta_{i}, i=1, \ldots, 7$, ou, o que é e quivalente, geramos 7 observações segundo uma distribuição $B\left(m_{i}, \theta_{i}\right)$, isto é, binomial com indice $m_{i}$ e probabilidade de sucesso $\theta_{i}(* *)$. Assim, geradas as amostras, passamos à fase de estimação, ou seja, calculamos as estimativas dos parâmetros $\theta_{1}, \ldots, \theta_{n}$ segundo os estimadores de (1) a (6) citados acima. Para os estimadores (2) e (5) foi utilizado o método iterativo de Newton Raphson (ver (2.2) e (2.3) para $\sigma^{2}$ conhecida e (2.5) e (2.6) para $\sigma^{2}$ desconheci da). Uma listagem do programa, que realiza este método bem como sua saída encontram-se no Apêndice 2. Para os es timadores (4) e (6) baseados no método de Laplace foi feito também um programa de computador que se encontra no Apêndice 3 (e o método de Newton Raphson foi também utilizą do) $(* * * *)$.

(*) - Os mesmos estimadores serão utilizados na seção seguinte (Estimação no modelo de Poisson).

(**) - Foi utilizada a subrotina GGNML do IMSL (IMSL Library, 1979).

$(* * *)$ - Foi utilizada a subrotina GGBN do IMSL .

$(* * * *)$ - Foram utilizadas as subrotinas LINV2F e LINV3F do IMSL para fazer inversões de matrizes e cälculos de determinantes. 
Ainda cabe notar um detalhe importante. Quando consideramos $\sigma^{2}$ desconhecida, tomamos uma distribuição a priori $x^{2}$-invertida. Como já mencionado, esta depende dos parâmetros $\nu$ e $\lambda$ (ver as observações feitas a este respeito nas páginas 15 e 16) e se não há nenhum conhecimento a priori sobre $\sigma^{2}$, o ideal seria tomar $\nu=0$, porém isto conduziria a uma distribuição a posteriori imprópria. Então para evitar este problema, tomamos $\nu=0,1$ e escolhemos arbitrariamente o valor 2 para o parâmetro $\lambda$. Como $v$ é bas tante pequeno, este valor de $\lambda$ praticamente não influencia os resultados.

As estimativas pontuais baseadas nos estimadores de (1) a (6) encontram-se na Tabela 4.1.

O programa do Apêndice 2 ainda fornece outras informações relevantes além destas estimativas, isto é, forne ce a matriz de covariância a posteriori (se supusermos uma aproximação normal para a distrỉbuição a posteriori). Com base nesta matriz é possivel construir intervalos de credi biłidade (como descrito no Capítulo 1).

Passamos agora a analisar dois aspectos importantes dos resultados obtidos:

i) a convergência dos processos iterativos e ii) a plausibilidade das estimativas e a comparação entre elas. 
Tabela 4.1 - Estimativas de $\theta$ obtidas em amostras simuladas Modelo binomial

\begin{tabular}{|c|c|c|c|c|c|c|c|}
\hline \multirow[b]{2}{*}{$\Theta_{i}$} & \multirow[b]{2}{*}{$\mathrm{m}_{i}$} & \multirow[b]{2}{*}{ (1) } & \multicolumn{3}{|c|}{$\sigma^{2}$ conhecida } & \multicolumn{2}{|c|}{$\sigma^{2}$ desconhecida } \\
\hline & & & (2) & (3) & (4) & (5) & (6) \\
\hline 0,469 & 45 & 0,467 & 0,471 & 0,469 & 0,471 & 0,473 & 0,473 \\
\hline 0,268 & 34 & 0,206 & 0,242 & 0,244 & 0,243 & 0,256 & 0,250 \\
\hline 0,799 & 38 & 0,868 & 0,829 & 0,825 & 0,828 & 0,814 & 0,822 \\
\hline 0,530 & 44 & 0,636 & 0,627 & 0,626 & 0,626 & 0,622 & 0,625 \\
\hline 0,440 & 39 & 0,359 & 0,374 & 0,374 & 0,375 & 0,381 & 0,378 \\
\hline 0,598 & 37 & 0,595 & 0,587 & 0,587 & 0,587 & 0,584 & 0,586 \\
\hline 0,464 & 36 & 0,472 & 0,477 & 0,477 & 0,477 & 0,479 & 0,479 \\
\hline
\end{tabular}

(1) - Estimador usual (média amostra1).

(2) - Moda da distribuiçāo a posteriori (dada em (2.1)).

(3) - Média da distribuição a posteriori obtida através de uma aproximação para a função de verossimilhança (ver (2.4)).

(4) - Média aproximada da distribuição a posteriori calculada pelo método de Laplace (Seçāo 2.1.3).

(5) - Moda da distribuição a posteriori (Seção 2.1.2).

(6) - Média aproximada da distribuição a posteriori calculada pelo método de Laplace (Seção 2.1.3).

A convergência do processo iterativo de Newton Raphson foi considerada alcançada quando

$$
\left|\frac{\alpha_{i(k+1)}-\alpha_{i(k)}}{\alpha_{i(k)}}\right| \leqq 0,001, i=1, \ldots, n \text {, }
$$

onde $\alpha_{i(k)}$ é o valor de $\alpha_{i}$ obtido na k-ésima iteração do processo. 
Depois de aplicarmos o processo em muitas amostras simuladas, observamos que a convergência foi alcançada na to talidade dos casos quando o processo foi aplicado à obtenção das estimativas (2) e (4), isto é, a estimativa modal e a ba seada no método de Laplace, ambas supondo $\sigma^{2}$ conhecida. Em alguns casos, na obtenção da estimativa modal (5) supondo $\sigma^{2}$ desconhecida, a convergência não foi alcançada. No entanto, notamos que se o número de grupos não for muito grande (5 ou 6) e o tamanho das amostras for razoavelmente grande (superior a 50) a convergência é alcançada em praticamente todos os casos.

Cabe ainda fazer um comentário a respeito do nūmero de iterações necessárias à convergência do processo. Levando em conta que este número pode depender dos valores iniciais $\alpha_{i(0)}, i=1, \ldots, n$, procuramos escolher estes valores de forma conveniente. Nos processos iterativos de obtenção das estimativas modais, tomamos $\alpha_{i(0)}=\log \left(\Theta_{i(0)} /\left(1-\Theta_{i(0)}\right)\right.$ onde $\theta_{i(0)}$ é a média da i-ésima amostra, exceto nos casos em que $\Theta_{i(0)}=0$ ou $\Theta_{i(0)}=1$. Quando isto ocorreu, tomamos $\theta_{i(0)}=0,001$ ou $\theta_{i(0)}=0,999$. No processo iterativo de obtenção das estimativas baseadas no método de Laplace, tomamos como valores iniciais $\alpha_{i(0)}, i=1, \ldots, n$, os valores obtidos na última iteração do processo de obtenção das estimativas modais. Assim procedendo, obtivemos a convergência rapidamente (em geral, em 3 ou 4 iterações).

Passemos agora a analisar as estimativas de $\Theta_{1}, \ldots, \theta_{n}$ obtidas pelos diversos métodos. Lembremos que alguns deles produzem estimativas para $\alpha_{1}, \ldots, \alpha_{n}$ e não para 
$\Theta_{1}, \ldots, \theta_{n}$ diretamente. Neste caso, calculamos $\tilde{\Theta}_{i}=e^{\tilde{\alpha}_{i}} /\left(1+e^{\tilde{\alpha}_{i}}\right), i=1, \ldots, n$, onde denotamos, genericamente, $\tilde{\alpha}_{i}$ como estimativa de $\alpha_{i}$ e tomamos $\tilde{\theta}_{i}$ como estimativa de $\Theta_{i}$. Voltemos então à Tabela 4.1 para analisar os resultados obtidos. Comparando simultaneamente as colunas (2) a (6), correspondentes às estimativas Bayesianas, com a coluna (1), correspondente às estimativas usuais, observamos que as Bayesianas são razoavelmente próximas das usuais. Além dis to, é possivel observar a "contração" nas estimativas Bayesi anas (como mencionado na pg. 14) . Observe-se, por exemplo, as diferenças entre a maior e a menor estimativas das colunas (1) e (2). Na coluna (1) obtém-se 0,868-0,206=0,662 e na coluna (2), 0,829-0,242=0,587 (menor do que 0,662). Daí vemos que, de fato, as estimativas modais (coluna (2)) foram "contraídas". Agora, comparando entre si as colunas (2), (3) e (4), correspondentes ao caso em que $\sigma^{2}$ é conhecida, observamos que os três métodos aproximados de estimação prọ duzem resultados bastante próximos. Lembrando que as estimativas da coluna (3) são obtidas facilmente sem necessidade de processo iterativo, temos então um método rápido e simples de estimação Bayesiana aproximada (no caso em que $\sigma^{2}$ é conhecida). As colunas (5) e (6), correspondentes ao caso em que $\sigma^{2}$ é desconhecida, também se mostram bastante parecidas. Resta ainda comparar as colunas (2) e (5) e também (4) e (6). O interesse nestas comparações é devido à seguin te pergunta: O fato de não se conhecer a variância $\sigma^{2}$ exerce grande influência nas estimativas? A resposta a esta per gunta parece ser negativa, pois em muitos casos analisados esta influência mostrou-se pequena. 


\subsection{Estimação no modelo de Poisson}

Nesta seção procuramos ilustrar alguns resultados apresentados sobre estimação no modelo de Poisson (Seção 2.2). O procedimento seguido aqui é absolutamente análogo ao da seção anterior.

Para gerar $\alpha_{1}, \ldots, \alpha_{n}$ fixamos $n=7, \quad \mu=1$ e $\sigma^{2}=1$ e geramos 7 observações independentes segundo a distribui ção $N(1,1)$. A partir destas observações calculamos $\theta_{i}=e^{\alpha}$, $i=1, \ldots, 7$, e geramos 7 amostras (de tamanhos $m_{i}$ pré-fixados) segundo uma distribuição de Poisson com média $\theta_{i}, i=1, \ldots ., 7^{(*)}$. Foram feitos programas de computador para gerar as amostras e calcular as estimativas (ver Apêndice 4 e Apêndice 5). A Tabela 4.2 mostra os resultados obtidos.

O processo iterativo de Newton Raphson, utilizado para obtenção das estimativas modais e as baseadas no método de Laplace, mostrou-se bastante eficiente no sentido de que apresentou convergência rápida (em geral, em 3 ou 4 itera ções) em todos os casos em que o aplicamos.

Analisando a Tabela 4.2 e comparando simultaneamente as colunas (2) a (6), correspondentes às estimativas Baye sianas, com a coluna (1), correspondente às estimativas usuais, observamos que as Bayesianas são razoavelmente próximas das usuais, porém mais "contraidas". Comparando as colunas (2), (3) e (4), observamos que os três métodos aproximados de estimação supondo $\sigma^{2}$ conhecida) produzem resultados bastante próximos,

(*) - Foi utilizada a subrotina GGPOS do IMSL. 
Tabela 4.2 - Estimativas de $\Theta$ obtidas em amostras simuladas Modelo de Poisson

\begin{tabular}{c|c|c|c|c|c|c|c}
\hline \multirow{2}{*}{$\Theta_{i}$} & \multirow{2}{*}{$\mathrm{m}_{\mathbf{i}}$} & $(1)$ & \multicolumn{3}{|c|}{$\sigma^{2}$ conhecida } & \multicolumn{2}{c}{$\sigma^{2}$ desconhecida } \\
\cline { 4 - 8 } 2,818 & 40 & 3,025 & 3,021 & 3,022 & 3,023 & 3,022 & 3,023 \\
2,409 & 32 & 2,219 & 2,224 & 2,224 & 2,225 & 2,222 & 2,223 \\
4,131 & 36 & 4,417 & 4,402 & 4,402 & 4,404 & 4,406 & 4,407 \\
9,065 & 29 & 8,552 & 8,511 & 8,511 & 8,515 & 8,521 & 8,524 \\
1,620 & 39 & 1,974 & 1,982 & 1,982 & 1,982 & 1,980 & 1,980 \\
5,936 & 42 & 6,167 & 6,146 & 6,147 & 6,149 & 6,151 & 6,154 \\
0,246 & 30 & 0,200 & 0,275 & 0,289 & 0,277 & 0,258 & 0,257 \\
\hline
\end{tabular}

(1) - Estimador usual (média amostral).

(2) - Moda da distribuição a posteriori (dada em (2.7).

(3) - Média da distribuição a posteriori obtida através de uma aproximação normal para a função de verossimilhança (dada em (2.8)).

(4) - Média aproximada da distribuição a posteriori calculada pelo método de Laplace (Seção 2.2.3).

(5) - Moda da distribuição a posteriori (Seção 2.2.2).

(6) - Média aproximada da distribuição a posteriori calculada pelo método de Laplace (Seção 2.2.3).

sendo que as estimativas da coluna (3) são facilmente obtidas sem necessidade de processos iterativos. As colunas (5) e (6), correspondentes a $\sigma^{2}$ desconhecida, mostram-se também bastan te parecidas. Finalmente, comparaldo entre si as colunas (2) e (5) e também (4) e (6) vemos que o fato de não se conhecer a variância $\sigma^{2}$ parece, praticamente, não afetar as estimativas. 
4.4. Estimação no modelo normal

Nesta seção alguns resultados sobre estimação no modelo normal (Seção 2.3) são ilustrados numericamente. Especificamente, calculamos estimativas pontuais para os parâmetros $\theta_{1}, \ldots, \theta_{n}$ baseados nos seguintes estimadores:

(1) Estimador usual, isto é, a média amostral;

(2) Média da distribuição a posteriori apresentada em (2.9) supondo que as variâncias são conhecidas e

Moda da distribuição a posteriori apresentada em

(2.10) supondo que as variâncias são desconhecidas.

As amostras foram geradas da seguinte forma: gera mos $\alpha_{1}, \ldots, \alpha_{7}$ (o que equivale a gerar $\theta_{1}, \ldots, \theta_{7}$ já que $\theta_{i}=\alpha_{i}$ ) independentemente segundo uma distribuição $N(10,4)$. A seguir geramos 7 amostras aleatórias (de tamanhos mi pré-fixados) segundo uma distribuição $\mathrm{N}\left(\Theta_{i}, \sigma_{i}^{2}\right)$ (fixamos $\sigma_{1}^{2}=4, \sigma_{2}^{2}=5, \sigma_{3}^{2}=4$, $\left.\sigma_{4}^{2}=3, \sigma_{5}^{2}=4, \sigma_{6}^{2}=6, \sigma_{7}^{2}=5\right)$. Foram feitos programas de computador para gerar as amostras e calcular as estimativas (ver Apên dice 6). A Tabela 4.3 mostra os resultados obtidos.

o processo de Newton Raphson, utilizado para a obtenção das estimativas modais, mostrou-se extremamente efici ente pois apresentou convergência bastante rápida (em geral,em 2 iterações) na totalidade dos casos em que o aplicamos.

Analisando a Tabela 4.3 vemos que as estimativas Bayesianas (colunas (2) e (3)) são bem próximas das usuais 
(coluna (1)) porém mais "contraídas". Agora comparando entre si as colunas (2) e (3) observamos que o fato de não se conhecer as variâncias parece praticamente não afetar os resul tados.

Tabela 4.3 - Estimativas de $\Theta$ obtidas em amostras simuladas

Mode1o normal

\begin{tabular}{c|c|c|c|c}
\hline$\Theta_{i}$ & $\mathrm{~m}_{i}$ & $(1)$ & $\begin{array}{c}\text { Variâncias } \\
\text { conhecidas } \\
(2)\end{array}$ & $\begin{array}{c}\text { Variâncias } \\
\text { desconhecidas } \\
\text { (3) }\end{array}$ \\
\hline 10,287 & 25 & 10,679 & 10,663 & 10,671 \\
13,243 & 20 & 13,415 & 13,230 & 13,349 \\
11,264 & 22 & 11,563 & 11,507 & 11,532 \\
10,685 & 23 & 11,085 & 11,059 & 11,073 \\
9,742 & 30 & 9,956 & 9,966 & 9,961 \\
10,669 & 25 & 4,464 & 4,793 & 4,635 \\
& 21 & 10,693 & 10,669 & 10,677 \\
\hline
\end{tabular}

(1) - Estimador usual (média amostral).

(2) - Média da distribuição a posteriori (dada em (2.9)).

(3) - Moda da distribuiçāo a posteriori (dada em (2.10)).

4.5. Previsão em amostragem em dois estágios

Apresentaremos nas seções seguintes ilustrações nu彑 méricas de resultados discutidos no Capítulo 3. Trabalharemos com populações e amostras simuladas. Inicialmente, gera- 
remos $\alpha_{1}, \ldots, \alpha_{N}$ segundo uma distribuição normal com média $\mu$ e variāncia $\sigma^{2}$. A seguir, calcularemos $\Theta_{i}$ (lembrando que $\Theta_{i}=a^{\prime}\left(\alpha_{i}\right)$ ) e geraremos independentemente $N$ populações (ou grupos) de tamanhos $\mathrm{M}_{1}, \ldots, \mathrm{M}_{\mathrm{N}}$ segundo uma distribuição de Bernoulli com média $\Theta_{i}$. O processo será repetido posterior mente para as distribuições de Poisson e normal. Após gera das as populações, serão selecionadas aleatoriamente $n$ entre elas, o que corresponde ao primeiro estágio da amostragem. Em seguida, serão selecionados aleatoriamente $m_{i}$, ies, elementos em cada uma das populações sorteadas no primeiro estágio. Desta forma completa-se o segundo estágio da amos tragem.

Nosso interesse será prever o total populacional

$$
T=\sum_{i=1}^{N} \sum_{j=1}^{M_{i}} Y_{i j}
$$

\subsubsection{Previsão no modelo binomial}

Nesta seção ilustramos numericamente diversos resultados obtidos no Capítulo 3 relativos a previsão no mo delo binomial. Especificamente, calculamos previsões do to tal populacional baseados em previsores da forma:

$$
\sum_{i \in s} \sum_{j \in s_{i}} y_{i j}+\sum_{i \in s} \sum_{j \notin s} \tilde{\theta}_{i}+\sum_{i \notin s} \sum_{j=1}^{M_{i}} \tilde{\Theta}_{i},
$$

onde $\tilde{\Theta}_{i}$ denota, genericamente, qualquer estimador de $\Theta_{i}$, $i=1, \ldots, N$. Utilizamos os seguintes estimadores: 
(1) Estimador usual, isto é,

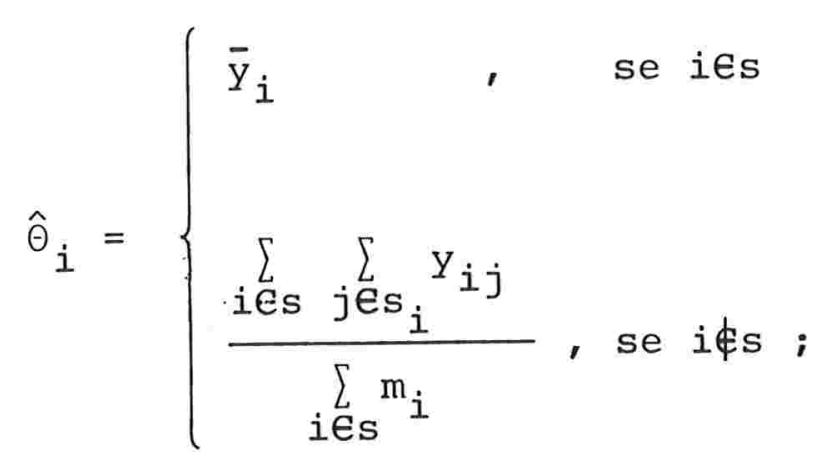

(2)

Moda da distribuição a posteriori, supondo $\sigma^{2}$ coㅇ nhecida;

Média da distribuição a posteriori aproximada obtida através de uma aproximação para a função de verossimiIhança, supondo $\sigma^{2}$ conhecida;

Média aproximada da distribuição a posteriori cal culada pelo método de Laplace, supondo $\sigma^{2}$ conhecida; Moda da distribuição a posteriori supondo $\sigma^{2}$ desconhecida e Média aproximada da distribuição a posteriori cal culada pelo método de Laplace supondo $\sigma^{2}$ desconhecida.

O processo de geração dos dados é o seguinte: ge ramos $\alpha_{1}, \ldots, \alpha_{10}$ independentemente segundo uma distribuição $\mathrm{N}(0 ; 0,2) ;$ calculamos $\theta_{i}=e^{\alpha_{i}} /\left(1+e^{\alpha}\right), i=1, \ldots, 10$ e geramos 10 populações (grupos) de tamanhos $M_{i}, i=1, \ldots, 10$, pré-fixados, segundo uma distribuição de Bernoulli com média $\Theta_{i^{\prime}}$ em seguida, selecionamos aleatoriamente 5 grupos, dos quais se 
lecionamos amostras aleatórias de tamanhos $\mathrm{m}_{i^{\prime}}$ i $\mathrm{es}$, também pré-fixados. O Apêndice 7 mostra os dados gerados.

$$
\text { Para o cálculo das estimativas de (2) a (6) acima }
$$

são necessários processos iterativos. O método de Newton Raphson foi utilizado (como explicado no Capitulo 3). Para a realização do método foram utilizadas as mesmas subrotinas dos programas mencionados na seção 4.2 (subrotinas BINCON e BINDES do Apênaice 3 e LABICO e LABIDE do Apênaice 4). Para tal convencionamos que para todo ies, isto é, pa ra todo grupo não selecionado, $y_{i}$ e $m_{i}$ são iguais a zero(*). A Tabela 4.4 mostra as previsões do total populacional base ados nos diversos estimadores.

$$
\begin{aligned}
\text { Tabela } 4.4 \text { - } & \text { Previsões do total populacional obtidas em populações e } \\
& \text { amostras simuladas } \\
& \text { Modelo binomial }
\end{aligned}
$$

\begin{tabular}{|c|c|c|c|c|c|c|}
\hline \multirow{2}{*}{$\begin{array}{l}\text { Total } \\
\text { Populacional }\end{array}$} & \multirow{2}{*}{ (1) } & \multicolumn{3}{|c|}{$\sigma^{2}$ conhecida } & \multicolumn{2}{|c|}{$\sigma^{2}$ desconhecida } \\
\hline & & (2) & (3) & (4) & (5) & (6) \\
\hline 1969 & 1709 & 1727 & 1733 & 1714 & 1719 & 1761 \\
\hline
\end{tabular}

As colunas de (1) a (6) correspondem às previsōes baseadas nos estimadores de (1) a (6) citados na página anterior.

(*) - Outra forma de encontrar as estimativas modais pelo método de Newton Raphson ë entrar nas subrotinas BINCON e BINDES com as informações sobre $m_{i}$ e $y_{i}$ somente dos grupos selecionados e calcular a stimatí va de $\alpha_{i}$, iфs, pela média das estimativas dos $\alpha_{i}$ 's dos grupos selecionados. Na realidade, è necessário fazer uma pequena modificação na subrotina BINDES: no cạ̄lculo de $\sigma_{(\mathrm{k})}^{2}$ o denominador deve ser subs tituido por ANI+NTOTAL-1 onde NTOTAL seria uma entrada da subrotina BINDES e indicaria o número total de grupos (selecionados ou não). 
Comparando as colunas (2) a (6) com a coluna (1) vemos que as previsões Bayesianas são razoavelmente próximas da usual. Agora, comparando entre si as colunas (2), (3) e (4) observamos que os três métodos de previsão Bayesiana (supondo $\sigma^{2}$ conhecida) produzem resultados bastante próximos, sendo que a previsão da coluna (3) é facilmente obtida sem necessidade de processos iterativos. Da mes ma forma, as previsões obtidas supondo $\sigma^{2}$ desconhecida mostram-se também muito próximas. Finalmente, comparando entre si as colunas (2) e (5) e também (4) e (6) vemos que o fato de não se conhecer as variâncias parece quase não afe tar os resultados.

Quanto à convergência dos processos iterativos, cabe fazer algumas observações. Em todos os casos em que aplicamos o método de Newton Raphson para obtenção das estimativas modais com $\sigma^{2}$ conhecida, a convergência foi obti da rapidamente (em geral, em 3 ou 4 iterações). Porém o mesmo sucesso não foi obtido quando supusemos $\sigma^{2}$ desconhecida. Em diversos casos a convergência não foi alcançada. No entanto, nas situações em que o número total de grupos e o de grupos selecionados não são muito grandes (por exem plo, 10 grupos dos quais foram selecionados 5) e os tamanhos das amostras são grandes (superiores a 50), a convergência é, na grande maịoria dos casos, alcançada rapidamen te. Na aplicação do processo iterativo para obtenção das estimativas baseados no método de Laplace, a convergência foi alcançada em praticamente todos os casos em que o apli 
camos, porém é bom lembrar que este só pode ser utilizado se as estimativas modais tiverem sido calculadas.

\subsubsection{Previsão no modelo de Poisson}

Nesta seção fazemos ilustrações numéricas de resultados do Capítulo 3 referentes a previsão do total populacional no modelo de Poisson. Seguimos um procedimento aná logo ao utilizado na seção anterior.

$$
\text { O Apêndice } 8 \text { mostra os dados gerados e a Tabela }
$$

4.5 mostra as previsões do total populacional baseados nos diversos estimadores.

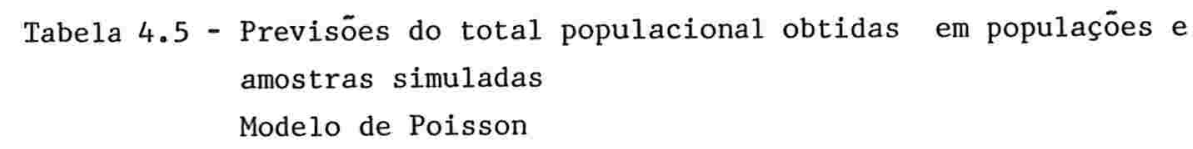

\begin{tabular}{c|c|c|c|c|c|c}
\hline \multirow{2}{*}{$\begin{array}{l}\text { Total } \\
\text { Populacional }\end{array}$} & \multirow{2}{*}{$(1)$} & \multicolumn{3}{|c|}{$\sigma^{2}$ conhecida } & \multicolumn{2}{c}{$\sigma^{2}$ desconhecida } \\
\cline { 3 - 7 } & & $(2)$ & $(3)$ & $(4)$ & (5) & $(6)$ \\
\hline \multirow{2}{*}{15582} & 17016 & 15468 & 15474 & 16386 & 15692 & 15773 \\
\hline
\end{tabular}

As colunas (1) a (6) correspondém às previsōes baseadas nos estimadores (1) a (6) citados na página 80 .

o processo iterativo de Newton Raphson, utilizado para o cálculo das estimativas modais e as baseadas no método de Laplace, mostrou-se bastante eficiente no sentido de que apresentou convergência rápida na totalidade dos casos em que o aplicamos (em geral em 3 ou 4 iterações). 
Comparando entre si as colunas (2) e (3) da Tabela 4.5 notamos que estas apresentam resultados bastante pró ximos, sendo que a previsão dada na coluna (3) é obtida facilmente sem necessidade de processo iterativo. Agora, compa rando as colunas (2) e (4) observamos que a previsão obtida pelo método de Laplace está um pouco afastada da previsão baseada nas estimativas modais (ambos supondo $\sigma^{2}$ conhecida), por outro lado, ao se comparar as colunas (5) e (6) correspondentes a $\sigma^{2}$ desconhecida, vemos que as previsões são bem próximas.

4.5.3. Previsão no modelo normal

Nesta seção ilustramos numericamente alguns resultados referentes a previsão do total populacional no modelo normal (Seção 3.4.3) . Especificamente, fazemos previsões baseadas nos seguintes estimadores de $\theta_{i}, i=1, \ldots, N$ :

Estimador usual;

Média da distribuição a posteriori supondo que as variâncias são conhecidas;

Moda da distribuição a posteriori supondo que as variâncias são desconhecidas.

O programa mencionado na seção 4.4 (Apêndice 6) foi utilizado, porém com algumas modificações. O Apêndice 9 mostra os dados gerados e a Tabela 4.6 mostra as previsões do total populacional baseadas nos estimadores de (1) a (3) acima. 
Tabela 4.6 - Previsōes do total populacional obtidas em populaçōes e amostras simuladas Modelo normal

\begin{tabular}{c|c|c|c}
\hline $\begin{array}{l}\text { Total } \\
\text { Populacional }\end{array}$ & $(1)$ & $\begin{array}{c}\text { Variāncias } \\
\text { conhecidas } \\
(2)\end{array}$ & $\begin{array}{c}\text { Variāncias } \\
\text { desconhecidas } \\
(3)\end{array}$ \\
\hline $65.171,0$ & $65.721,7$ & $65.274,2$ & $65.335,6$ \\
\hline
\end{tabular}

As colunas (1) a (3) correspondem ás previsōes baseadas nos estimadores (1) a (6) citados na página 80 .

Comparando as colunas (2) e (3) da Tabela 4.6, com

a coluna (1) vemos que as previsões Bayesianas são razoavel mente próximas da usual. Além disto, comparando entre si as colunas (2) e (3) vemos que o fato das variâncias não serem conhecidas parece não afetar muito a previsão (este comportamento foi observado em diversos exemplos que analisamos). Além disto, o processo iterativo mostrou-se bastante eficiente no sentido de que apresentou convergência rápida (em geral, em 2 iterações). 
APENDICE 1

CÁLCULO DOS MOMENTOS A POSTERIORI APROXIMADOS PELO MÉTODO DE LAPLACE

Tierney e Kadane (1986) propõem aproximações para médias, variâncias e covariâncias a posteriori de funções positivas e contínuas dos parâmetros. Estas aproximações são baseadas no método de Laplace para integrais descrito em De Bruijn (1958).

Seja $\mathrm{g}(\cdot)$ uma função contínua e positiva definida no espaço paramétrico. A média a posteriori de $g(\theta)$ pode ser escrita como

$$
E[g(\theta) / \mathbf{Y}]=\frac{\int g(\theta) L(\theta / Y) \pi(\theta) d \theta}{\int L(\theta / \mathbf{Y}) \pi(\theta) d \theta}
$$

onde L é a função de verossimilhança, $\pi$ é a função de densidade a priori do parâmetro $\theta$ e $\mathbf{y}$ é o vetor de observações.

$$
\begin{aligned}
& \text { Seja } \\
& L(\theta / \mathbf{Y})=(\log \pi(\theta)+\log L(\theta / \mathbf{Y})) / \mathrm{m}
\end{aligned}
$$

e

$$
L *(\Theta / \mathbf{Y})=(\log g(\Theta)+\log \pi(\Theta)+\log L(\Theta / \mathbf{Y})) / m,
$$

onde m é o tamanho da amostra. A expressão (A.1) pode ser escrita como

$$
E[g(\theta) / y]=\frac{\int \exp \left\{m L^{*}(\theta / \mathbf{Y})\right\} d \theta}{\int \exp \{m L(\theta / \mathbf{Y})\} d \theta} .
$$


Seja $\tilde{\Theta}$ a moda de $L$ e $\gamma^{2}=-1 / L "(\tilde{\Theta} / \mathbf{Y})$. O método de Laplace produz a seguinte aproximação para o denominador de (A.1):

$$
\begin{aligned}
\int \exp \{m L(\Theta / \mathbf{Y})\} & \cong \int \exp \left\{m L(\tilde{\Theta} / \mathbf{Y})-m \frac{(\Theta-\tilde{\Theta})^{2}}{2 \gamma^{2}}\right\} d \theta \\
& =\frac{\sqrt{2 \pi} \gamma}{\sqrt{m}} \exp \{m L(\tilde{\Theta} / \mathbf{Y})\} \cdot
\end{aligned}
$$

Analogamente, denotando por $\Theta^{*}$ a moda de $L^{*}$ e $\gamma^{* 2}=-1 / L^{*} "\left(\Theta^{*} / \mathbf{y}\right)$, obtem-se a seguinte aproximação para o numerador de (A.1):

$$
\frac{\sqrt{2 \pi} \gamma^{*}}{\sqrt{m}} \exp \left\{m L^{*}\left(\Theta^{*} / y\right)\right\}
$$

Assim, calculando o quociente das duas aproximações, tem-se uma aproximação para a média a posteriori de $g(\theta)$ :

$$
\hat{E}[g(\Theta) / \mathbf{Y}]=\frac{\gamma *}{\gamma} \exp \left\{m\left[L^{*}\left(\theta^{*} / \mathbf{Y}\right)-L(\tilde{\Theta} / \mathbf{y})\right]\right\}
$$

ou equivalentemente,

$$
\hat{E}[g(\Theta) / \mathbf{Y}]=\frac{\gamma^{*}}{\gamma} \frac{g\left(\Theta^{*}\right) \pi\left(\Theta^{*}\right) L\left(\Theta^{*} / \mathbf{Y}\right)}{\pi(\tilde{\Theta}) L(\tilde{\Theta} / \mathbf{Y})} .
$$

No caso multivariado, denotando por $\theta$ o vetor de parâmetros, temos a seguinte aproximação:

$$
\hat{E}[g(\theta) / \mathbf{Y}]=\left(\frac{\operatorname{det} \mathbf{D}^{*}}{\operatorname{det} \mathbf{D}}\right)^{1 / 2} \frac{g\left(\boldsymbol{\theta}^{*}\right) \pi\left(\boldsymbol{\theta}^{*}\right) L\left(\boldsymbol{\theta}^{*} / \mathbf{Y}\right)}{\pi(\tilde{\theta}) L(\tilde{\boldsymbol{\theta}} / \mathbf{Y})}
$$

onde $\tilde{\boldsymbol{\theta}}$ e $\tilde{\boldsymbol{\theta}}^{*}$ são as modas de $\pi(\boldsymbol{\theta}) L(\boldsymbol{\theta} / \mathbf{y})$ e $g(\boldsymbol{\theta}) \pi(\boldsymbol{\theta}) L(\boldsymbol{\theta} / \mathbf{Y})$ respectivamente, D é a inversa da matriz cujo elemento $(i, j)$ é 


$$
-\left.\frac{\partial^{2} \log (\pi(\theta) L(\theta / \mathbf{y}))}{\partial \theta_{i} \partial \theta_{j}}\right|_{\theta=\theta^{*}}
$$

e $D^{*}$ é a inversa da matriz cujo elemento $(i, j)$ é

$$
-\left.\frac{\partial^{2} \log (g(\theta) \pi(\theta) L(\theta / \boldsymbol{y}))}{\partial \Theta_{i} \partial \theta_{j}}\right|_{\theta=\theta^{*}}
$$

$i, j=1, \ldots, n$.

Para obter uma aproximação para a variância a posteriori de $g(\theta)$ basta tomar

$$
\operatorname{Var}[g(\theta) / \mathbf{y}]=\hat{E}\left[g^{2}(\theta) / \mathbf{y}\right]-\hat{E}^{2}[g(\theta) / \mathbf{Y}]
$$

e, da mesma forma, para encontrar uma aproximação para a covariância a posteriori de $g(\boldsymbol{\theta})$ e $\mathrm{h}(\boldsymbol{\theta})$ toma-se

$$
\begin{aligned}
\hat{\operatorname{Cov}}[g(\theta), h(\theta) / \mathbf{Y}] & =\hat{\mathrm{E}}[g(\theta) h(\theta) \mid \mathbf{y}]- \\
& -\hat{E}[g(\theta) / \mathbf{Y}] \hat{\mathrm{E}}[\mathrm{h}(\theta) / \mathbf{Y}] .
\end{aligned}
$$

Como se pode constatar, o método é bastante simples, envolvendo somente algumas maximizações e derivações (no máximo até segunda ordem).

Tierney e Kadane (1986) observam que as aproximações são, em geral, tão acuradas e, em alguns casos, mais acuradas que as aproximações baseadas em expansões da função de verossimilhança até terceira orcem (quee, obviamente, requerem cálculo de derivadas de terceira ordem) e afirmam que a principal condição de regularidade necessária à aplicação do 
método é que a função de densidade a posteriori seja unimodal. Os autores analisam, em detalhes, as ordens das aproximações. OOO 
APÊNDICE 2 - Saída e listagem do programa que calcula as estimativas modais de $\boldsymbol{\theta}$. Modelo binomial 
?EEGIN JOR BINJMIAL

?CH. AFGE $=$ NE. WT ON $=$ R AP HSON

?CONPILE BINCMIAL FDFTHAN LIBFAFY

?FOFTFAA DAT A

\$SER ALTOBIND

\& $\triangle I N D=F F O M \quad E I H / I M S L / 2, B I A / I M S L / 3, E I E / I M S L / 4$

DCUBLE PRECISICN DSEED

C JMENS JON M(2:), Y (20), GALFA(20), GTETA(20), ALF \& (20), ALFG(2O)

KEAD $(3,1) \mathrm{N}, A M E D, S I G M A 2$. AITHAX,PFECI S, CSEFD

1 F[FMAT $(1 X, 12, F 3,0, F], 0,1 X, 12, F 5,2, F 3,3)$

KFITE 6,6$)$ N.ANED, SIGNAC, PFECIS, ATINAX, DSEED

4 FCFMAT " NUNETC DE GRUPOS ( $A)=0,12, \%$ MEDIA CA NORMAL (AMED) $="$, *F3.0,1," VARJANCI A DA NORMAL (SIGMAL) =",FB.4,1,

" "PKEEISAD DESEJADA (PRECIS) =",F7.3./"

* NUMEKO MAXIMO DE ITEFACDES (NITNAX) =", IE, /, SEMENTE = "E13.8)

FF.AD $(5,2) N,(M(I), I=1, N)$

2 FCKMAT $(*(1 X, I 2))$

$L=N+1$

CALL G GNHL (DSEED, L, ALF G)

CC $1 \mathrm{G} \quad I=2, \mathrm{~L}$

C ALF A $(I-1)=A L F E(I) * 5 O F T(S I G M A 2)+A M E D$

10 CCNTINUE

C. $501=1 \cdot N^{\prime}$

ETETAC I $)=E X P(G A L F A(I)) /(I \& E X P(G A L F A(1)))$

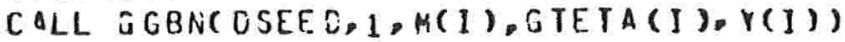

59 C CNT IVUE

ИFITE $(6,3) N,($ EALFA(I), GTETA(I),N(I),Y(I), I=1,N)

20 FCFMAT $(1 X, F 5,3,1 X, F 2.0)$

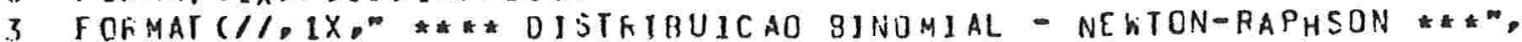
* / / " ALFA GERADO TETA GEPADO

$* *(3 X, F 8,4,12 X, 55,3,9 X, 13,8 X, 13,1), 11)$

AITMAX $=$ NI TMAX- 1

FIEAD $(3,5)$ IOPCAD

5 F CFMAT $(1 X, I 1)$

IF(IO?CAO.EQ.0) CALL BINCON(Y,M,N, SIGMA2,NITMCX,PFECIS)

IF(IOPCAÜ.EQ.1) CALL BINDES(Y,N,A,NITNAX,PFECIS)

$1 F\left(J O P C A_{0} \cdot N E .2\right)$ GO TO 70

CALL 3 INCON( $Y, M$, N, SIGMAZ, NITPAX, FFECIS)

CALL BINDES( $Y, M, N, N I T N A X, P F E C I S)$

70 STOP

END

SUBRUUTINE BINCON( $Y, M, N$, SIENA2, NITMAX, PFECIS)

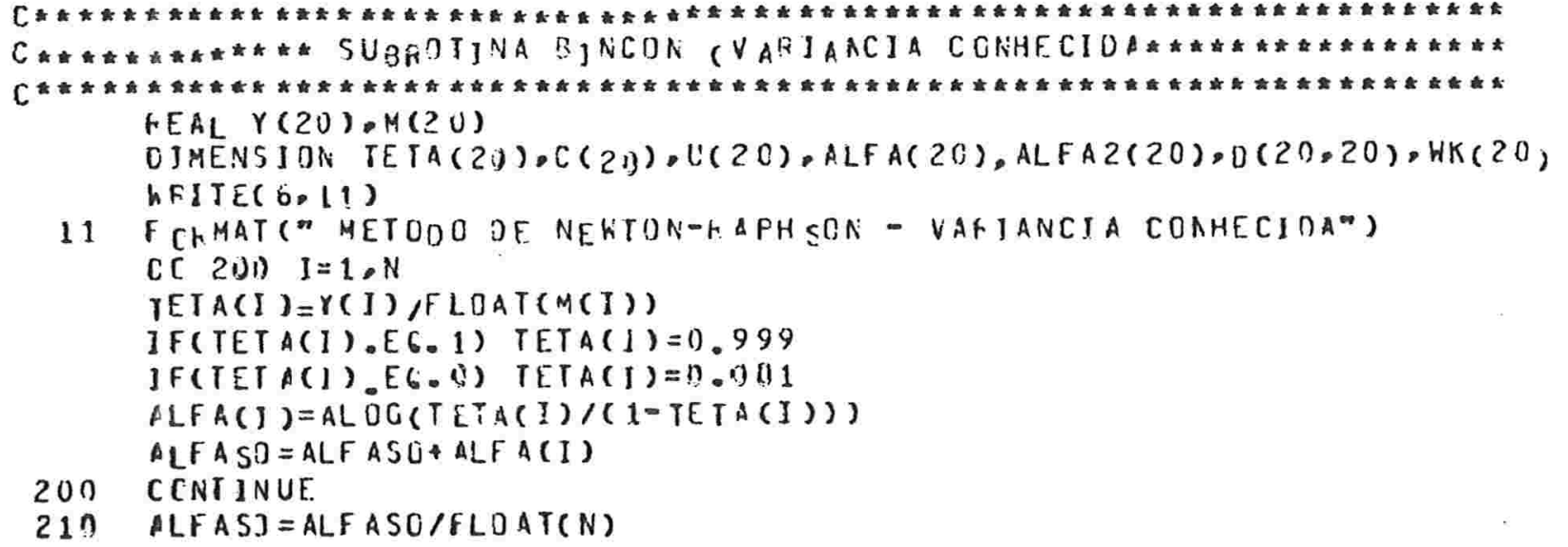




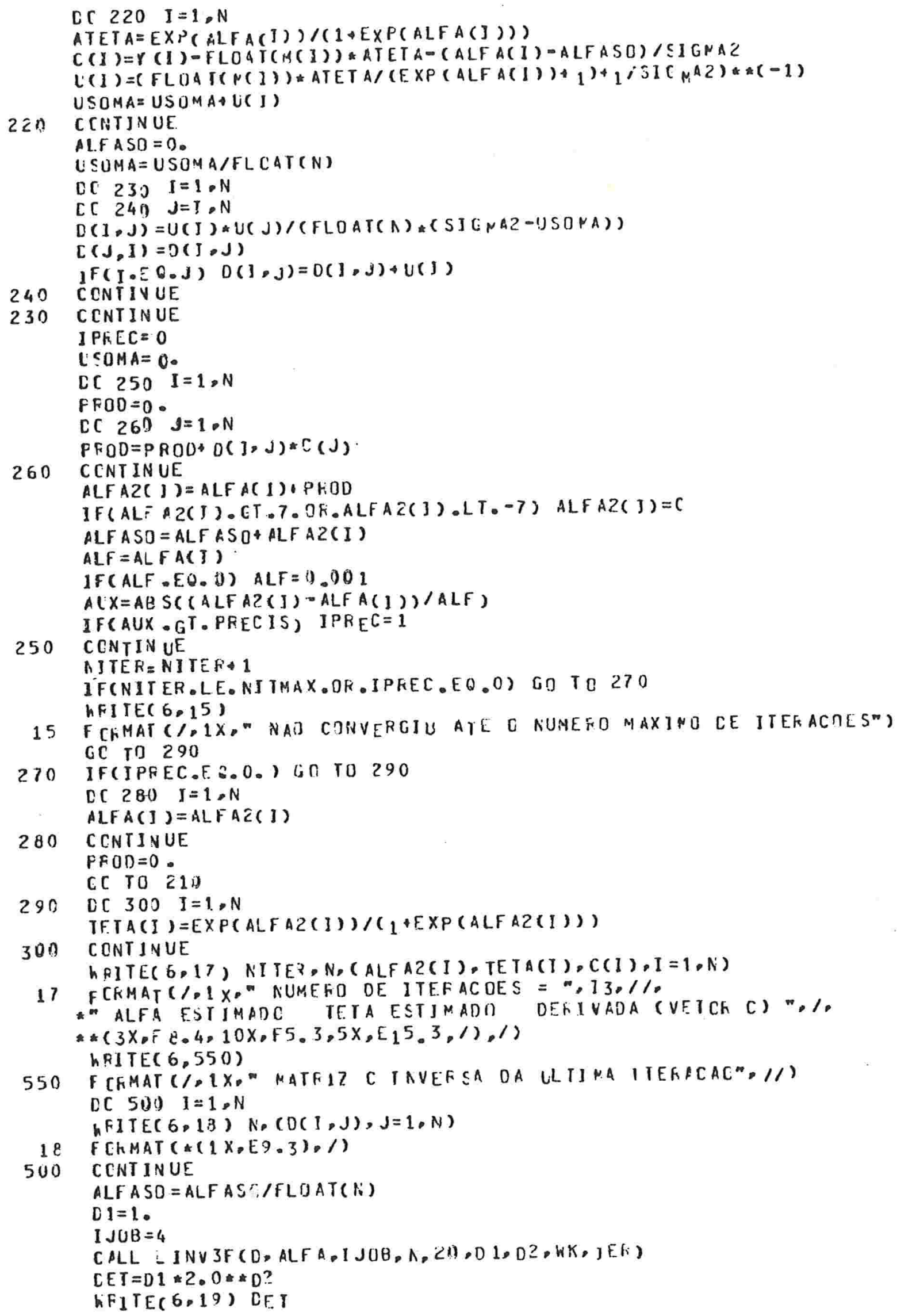


19 F[FMAT $(/ /, "$ DETEFM]NANTE DA NATRJZ C JNVEFSA = "E 15.8, $/)$ FETURN

END

SUBFOUTINE BINDES ( $Y, M, N D N I I N A X, P F E C I S)$

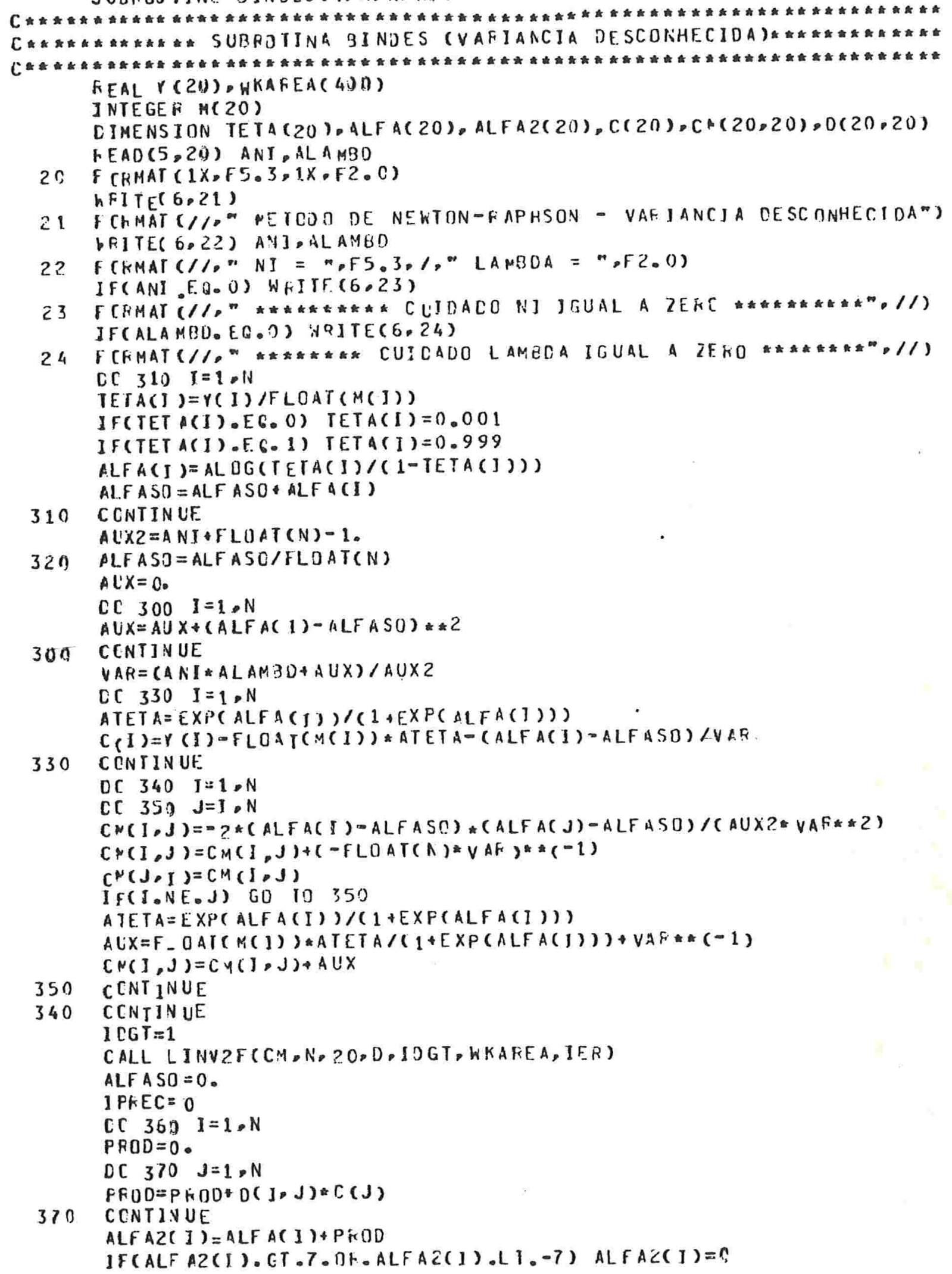




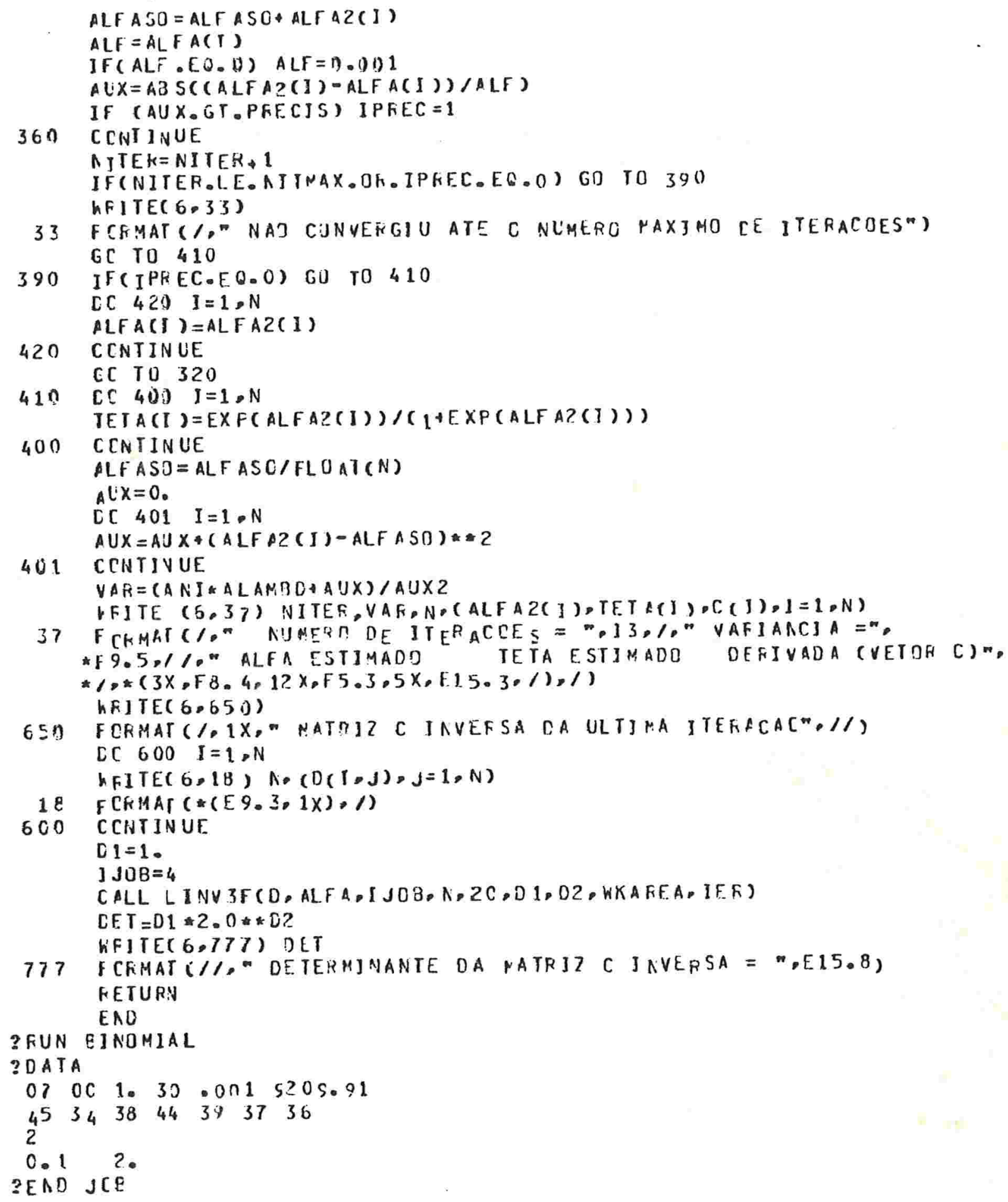


NUMERO DE. GRUPOS $(N)=7$

MEDIA DA NORMAL (AME)) $=0$.

VAFIANCIA DA NORMAL (SIGMAZ) = $1.0 \%$ OHO

PRECISAD DESEJAOA (PZECIS) $=0.001$

NUMERO MAXIMO DE ITERACOES (NITMAX) $=30$

SEMENTE $=.92099100 E$. 4

$\begin{array}{cccc}\text { *** UISTRIBUICAD } & \text { BINOMIAL - NEWTON-RAPHSON * * } \\ \text { ALFA GEFADD } & \text { TETA GERADO } & N & \gamma \\ -0.1227 & 0.469 & 45 & 21 \\ -1.0068 & 0.268 & 34 & 7 \\ 1.3315 & 0.799 & 33 & 33 \\ 0.1214 & 0.539 & 44 & 28 \\ -0.2409 & 0.440 & 39 & 14 \\ 0.3982 & 0.398 & 37 & 22 \\ -0.1423 & 0.464 & 36 & 17\end{array}$

MEIODO DE NEWTON-RAPISON - VARIANCIA CONHECTOA

NUMERO DE ITERACOES $=3$

$\begin{array}{ccc}\text { ALFA ESTIMADO } & \text { IEJA ESTIMADB } & \text { DERIJADA (JEIOR C) } \\ -0.1157 & 0.471 & -.176 E-07 \\ -1.1621 & 3.242 & -.170 E=03 \\ 1.5790 & 0.829 & .159 E-02 \\ 0.5173 & 3.627 & .304 E-06 \\ -0.5138 & 0.374 & -.656 E-077 \\ 0.3529 & 0.587 & .109 E=06 \\ -0.0917 & 0.477 & -.172 E=07\end{array}$

MATRIZ C INVERSA OA ULTIMA ITERACAO

\begin{tabular}{|c|c|c|c|c|c|c|}
\hline $.830 E=01$ & $-1 B 1 E-132$ & $-2 \because j E-n 2$ & $.116 E-D 2$ & $-13: E-\cap 2$ & $.132 E=02$ & $-132 E-32$ \\
\hline $.181 \mathrm{E}=02$ & $.141 E+00$ & $.34 P E=02$ & $.196 E=02$ & $.219 E=02$ & $.222 E-02$ & $.222 E-02$ \\
\hline $.206 E=02$ & $.347 E-122$ & $.161 E+00$ & $.222 E-122$ & $-248 E-\pi 2$ & $.252 E=72$ & $.252 E-02$ \\
\hline $.116 \mathrm{E}-\mathrm{J} 2$ & $.196 E=02$ & - $22 ? E=02$ & $.398 E=01$ & $.140 E=02$ & $.142 E=02$ & $.142 E-02$ \\
\hline $.130 E=02$ & $.219 E-02$ & $.249 E-122$ & $.14 \cap E-\cap 2$ & - IDAE $\triangle O O$ & $.159 E-\cap 2$ & $.159 E-n 2$ \\
\hline $.132 E=02$ & $.222 E=02$ & $.252 E=02$ & $.142 E=02$ & $.159 E=02$ & $.102 E \cdot 00$ & $.161 E=02$ \\
\hline$-132 E-02$ & $-222 E-102$ & $.252 E=0 ?$ & $.142 E-02$ & $.159 E-02$ & $.161 E-n 2$ & $.102 E+n$ \\
\hline
\end{tabular}

DETERMINANTE DA MATRLZ C INVERSA $=.17467768 E-05$ 
METODO DE NEWTON-FAPHSON - VARIANCIA DESCONHECIOA

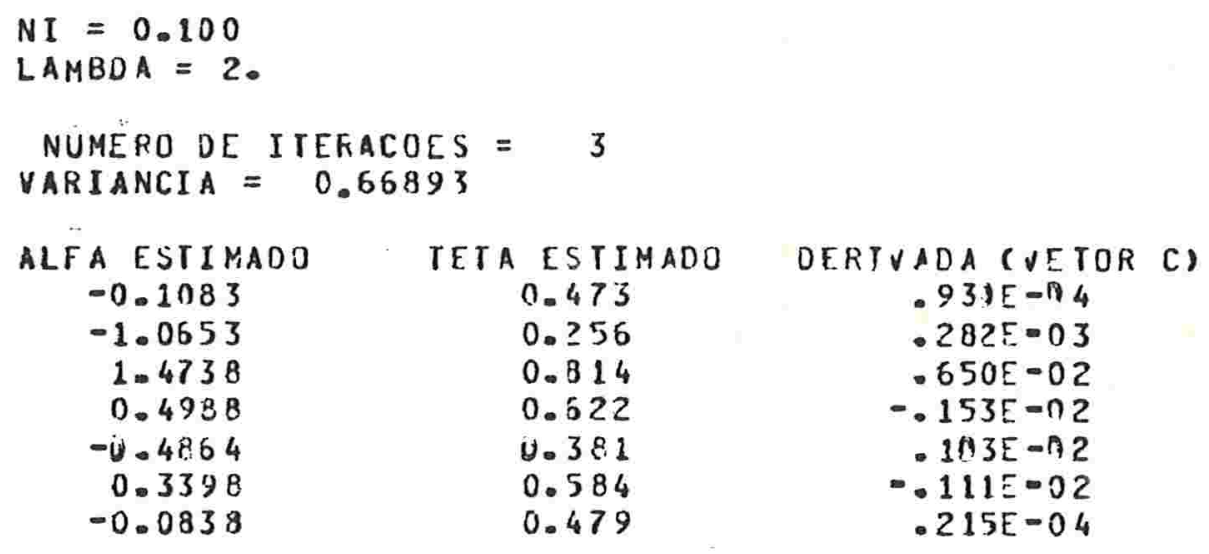




\section{APENDICE 3 - Saída e listagem do programa que calcula as estimativas de $\boldsymbol{\theta}$ ba- seadas no método de Laplace. Modelo binomial}




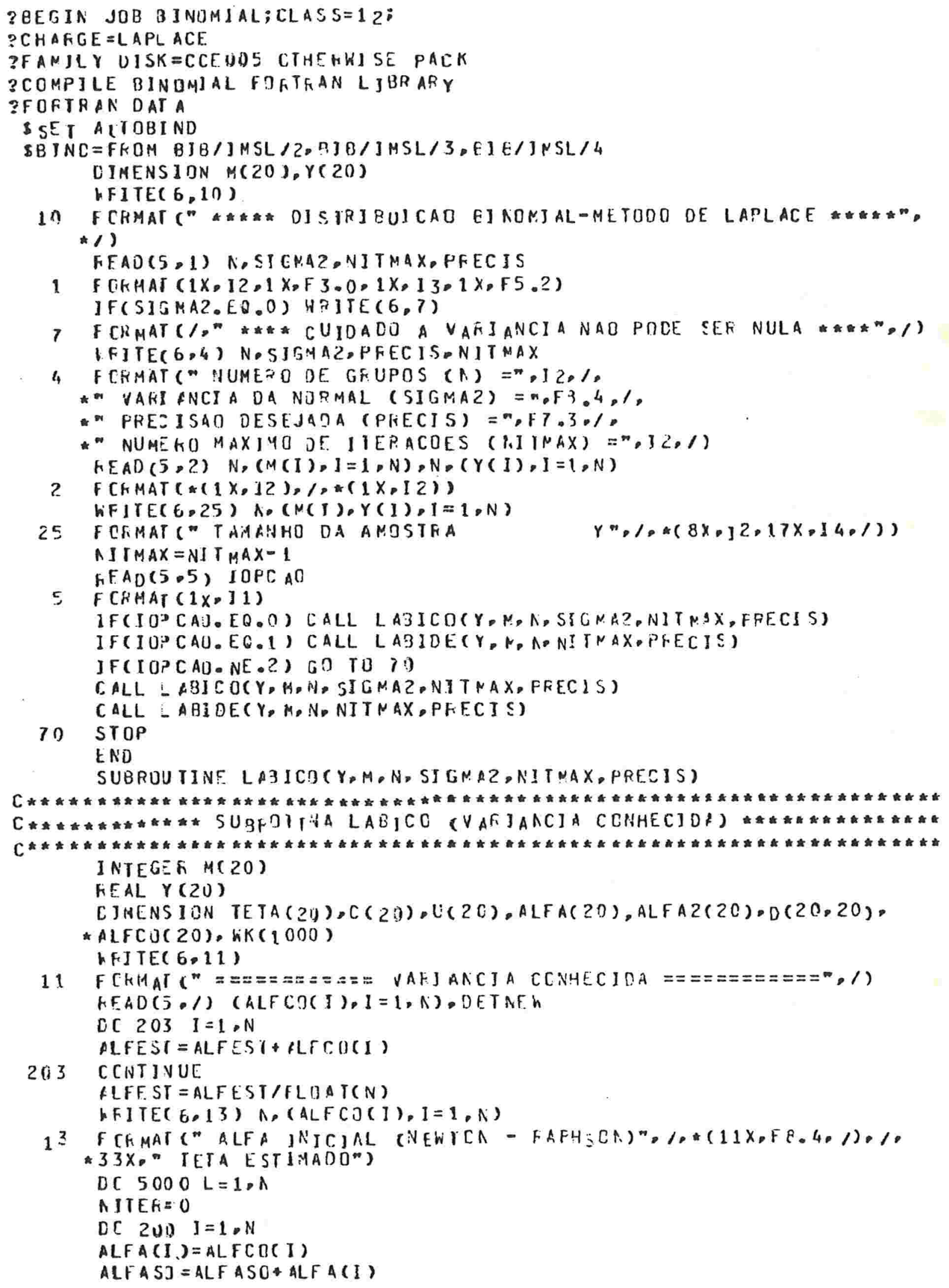


200 CENTINUE.

210 PLFASO = ALFASC/FLOAT(N)

CC $220 \quad I=1, N$

$A T E T A=\operatorname{EXP}(\operatorname{ALFA}(J)) /(1 \triangleleft \operatorname{EXP}(A L F A(J)))$

$\mathbb{C}(1)=0$.

IF(I. $\left.: G_{0} L\right) C(I)=1 . /(1 .+E X F(A L F A(I)))$

$C(J)=C(I)+Y(1)-F L D A T(M(I))$ A $Y E T A-(A L F A(I)-A L F \perp \leq 0) / 51 G M A 2$

P U X $=F L D A T(M(1))$

IF(I.EG.L) $M A \cup X=H A \cup X+1$

$U(1)=(M A U X * A \Gamma E T A /(1+E X P(\operatorname{ALFA}(1)))+1 / S 1 E K A 2) * \pm(-1)$

LSOMA $=$ USOMA+ C'( $B)$

220 CCNTINUE

ALFASO $=0$.

USOMA $=$ USOMA/FLOAT (N)

DC 231$] \quad I=1, N$

C[ $240 \quad]=1, N$

$C(I, J)=U(I) * U(J) /(F L O A T(N) *(\leq I G M A 2-115(3 N A))$

$[(3,1)=D(1, J)$

J $F\left(I \cdot E Q_{0} J\right) \quad D(I, j)=D(I, j)+U(1)$

240 CENTINUE

230 CCNT JNUE

J $P K E C=0$

L.SUMA $=0$.

CC $250 \quad I=1, N$

$P F D D=1$.

LC $26 ! J=1, N$

FFOD $=P K D O * 0(I, J) * C(J)$

260 CENT INUE

$A L F A Z(1)=A L F A(1), D R O D$

$A L F A S ?=A L F A S Q+A L F A Z(I)$

$A L F=A-F A(I)$

I $F(A L F \cdot E Q .0) \quad A L F=0.001$

$A(X=A B S(C A L A ?(I)-A L F A(I)) / A L F)$

IF (AUX,GT,FFECIS) IPFEC $=1$

250 CINTINUE

AIJEF:=NITEF+I

IF(NITER.LE.NITMAX,OF,IPFEC.EQ.0) GC TO 270

WFITE 6,15$) \mathrm{L}$

15 FCFMAT $(1,1 X, "$ NAD CDNVERGIU ATE C NUMEgO MAXING CE JTERACDES", $* \quad L=\cdots, 13)$

GE TO 290

230 IF(IPFEC.EG.0.) GO TO 29i)

C[ $2 \& 0 \quad]=1, N$

$A L F A(I)=A L F A 2(1)$

280 CCNTINUE

GC TO 210

$290 \quad$ E $1=1$.

$1 \mathrm{JOB}=4$

CALL - JNVZF(D, ALFA, JDB, N, 29, D 1, D2,WK, IE!)

CET $=D I * 2.0 * C ?$

ALFASO $=$ ALFASO/FLOAT(N)

AUX $=0$.

$A \cup \times 1=0$.

A $\mathrm{C} \times 3=0$.

$C\left[550^{\circ} \mathrm{J}=1, \mathrm{~N}\right.$

$A(X X=A U X+(-(A L F A 2(I)-A L F A S C) \Rightarrow 2)+(A L F C[(3)-A L F E S T)=2$

$A L X 1=A U X 1+Y(I) *(A L F A 2(I)-A L F C O(I))$

$A\left(X_{3}=A U X_{3}+M(I) *\left(A L B G(1+E X P(A L F A 2(J)))-A L U S_{1}(1+E X P(A L F C O(I)))\right.\right.$

$550 \quad$ CCNT INUE

$A(X=A U X /(2 * S I G M A 2)$ 
$E=S$ OKT $(D E T / D E T A E W)=E X P(A L F A Z(L)+A U X+A U \times 1-A U \times 3) /(1+E X P(A L F A Z(L)))$ WR] TE $(6,19)$ NITERDL, E

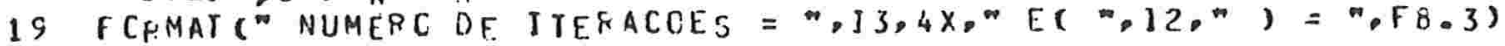

5000 CCNTINUE

FETUEV

$E A D$

SLBROU TINE LABIDE $(Y, M, N, M I T N A X, F F E C I S)$

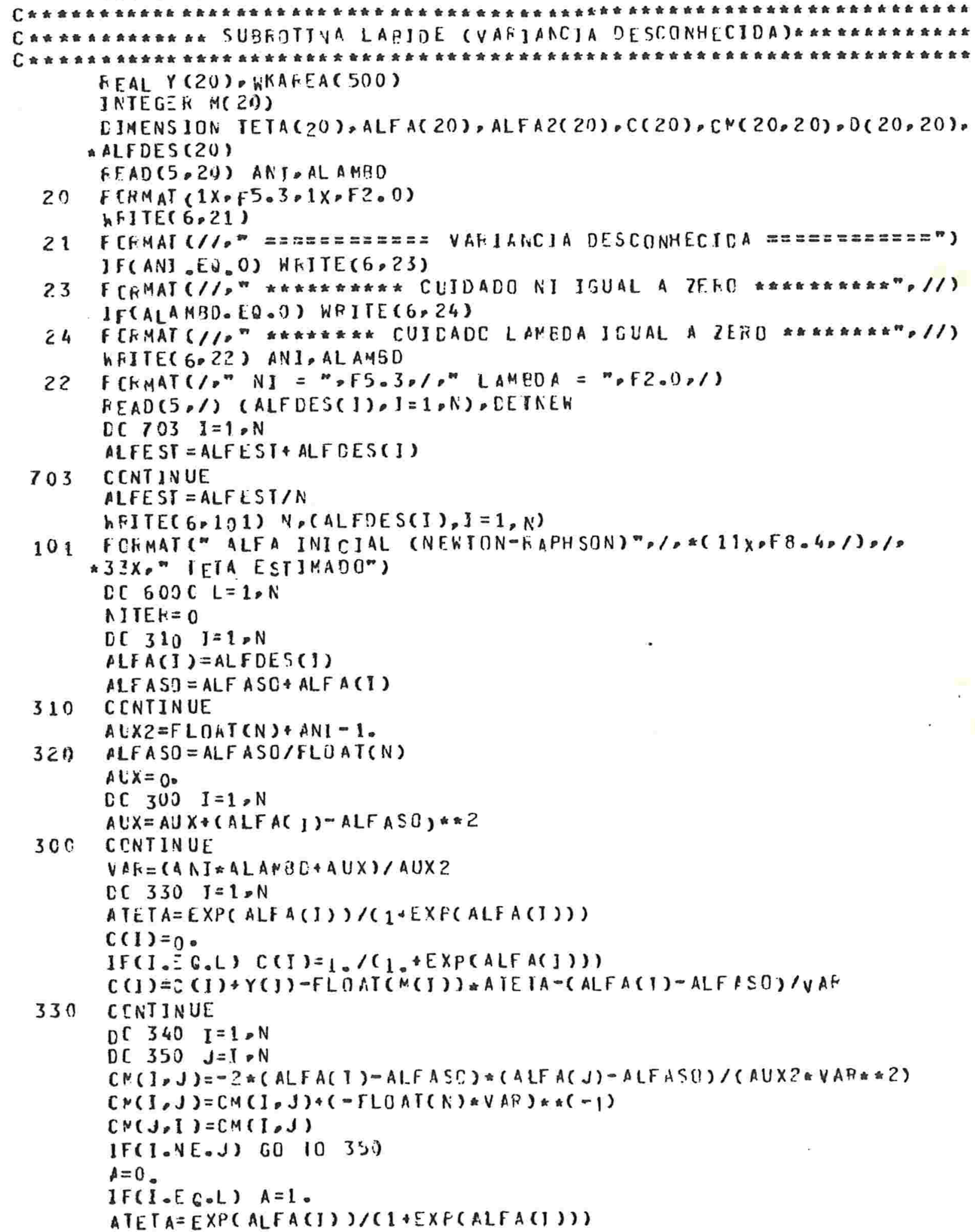




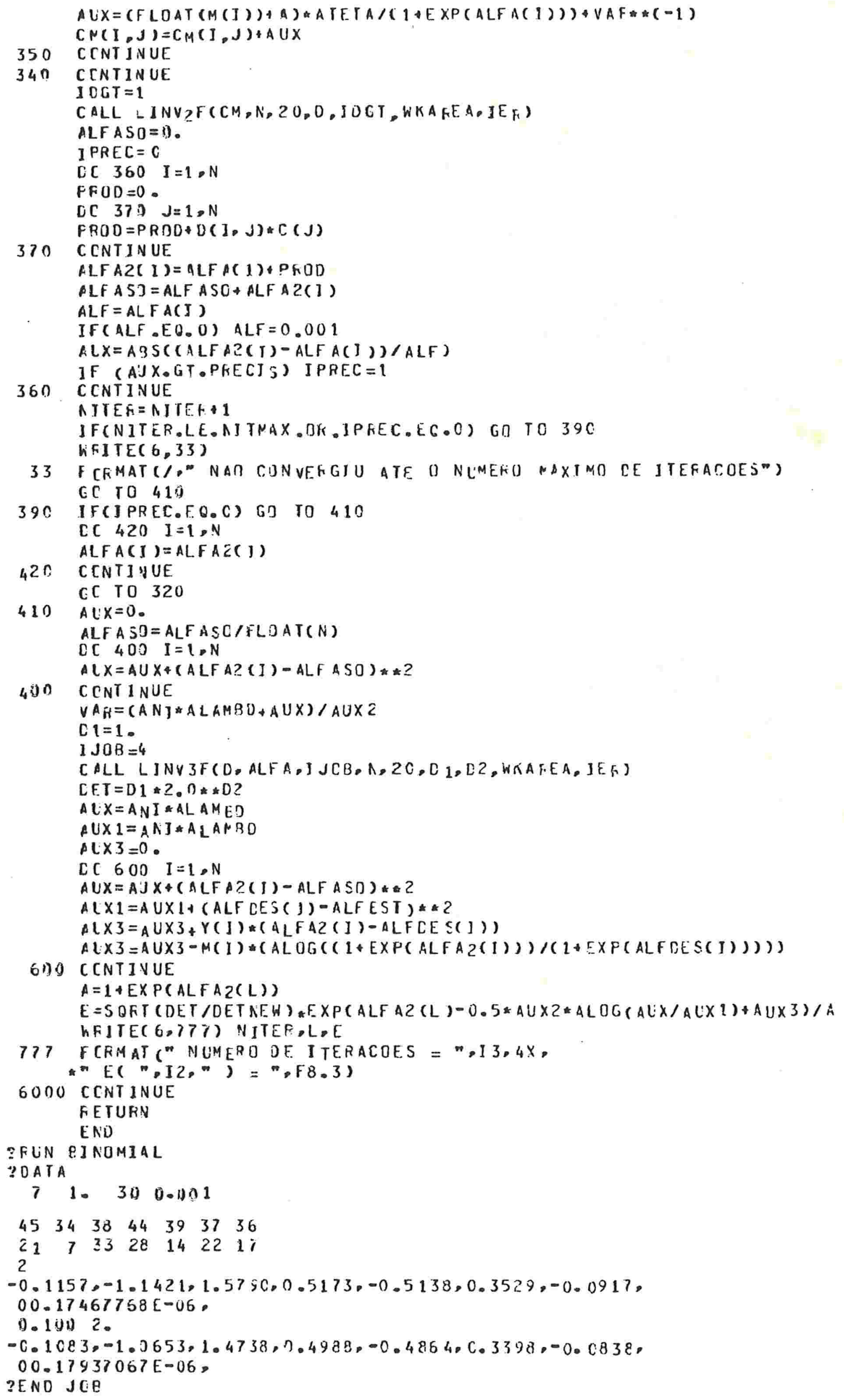


*** DISTRIBUICAD BINOMIAL-METODO DE LAPLACE \#\#

NUMERD DE GRUPOS $(N)=7$

$\checkmark A R I A N C I A$ DA NCRMAL (SIGMAZ) $=1.0 n O N$

PRECISAO DESEJADA (PZECJS) $=0.0 \% ?$

NUMERO MAXIMO DE ITERACDES (NITMAX) $=30$

$\begin{array}{cr}\text { TAMANHO DA AMOSTRA } & \gamma \\ 45 & 21 \\ 34 & 7 \\ 38 & 33 \\ 44 & 28 \\ 39 & 14 \\ 37 & 22 \\ 36 & 17\end{array}$

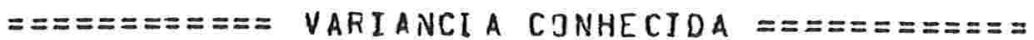

ALFA INICIAL (NEWTON - RAPHSON)

$$
\begin{array}{r}
-0.1157 \\
-1.1421 \\
1.5790 \\
0.5173 \\
-0.5138 \\
0.3529 \\
-0.0917
\end{array}
$$

\begin{tabular}{|c|c|c|c|c|c|c|c|c|}
\hline & & & & & & {$[E]$} & & STIMADO \\
\hline NUMERO & $D E$ & ITERACOES & & 2 & EC & 1 & ) & $=0.471$ \\
\hline NUMERO & $D E$ & ITERACOE S & $=$ & 3 & EC & 2 & ; & 0.243 \\
\hline NUMERD & $D E$ & ITERACOES & $=$ & 3 & Er & 3 & ) & 0.828 \\
\hline NUMERO & $D E$ & ITERACOES & $=$ & 3 & EC & 4 & ) & 626 \\
\hline NUMERO & $D E$ & I IEFACOES & $=$ & 3 & EC & 5 & ) & .375 \\
\hline NUAERO & $D E$ & ITERACOES & $=$ & 3 & Er & 6 & j & 0.587 \\
\hline NUMERO & $D E$ & ITERACOES & $=$ & 3 & Ec & 7 & ) & 0.477 \\
\hline
\end{tabular}

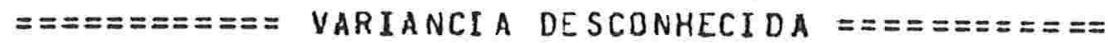

$N I=0.100$

$\angle A M B D A=2$.

ALFA INICIAL (NEWTON=RAPHSON)

$$
\begin{array}{r}
-0.1083 \\
-1.0653 \\
1.4738 \\
0.4988 \\
-0.4864 \\
0.3393 \\
-0.0838
\end{array}
$$

NUMERO DE ITERACOES = 3 NUMERO DE ITERACDES = 3 NUMERO DE ITERACOES $=3$ NUMERO DE ITERACOES = 3 NUMERO DE ITEFACOES = 3 NUMERO DE ITERACDES = 3 NURERO DE ITERACOES = 3

$\begin{array}{lll}\text { TETA } & \text { ESTIMADO } \\ E( & 1)= & 0.473 \\ E( & 2)= & 0.250 \\ E( & 3)= & 0.822 \\ E( & 4)= & 0.625 \\ E( & 5)= & 0.378 \\ E( & 6)= & 0.586 \\ E( & 7)= & 0.479\end{array}$


APÊNDICE 4 - Saída e listagem do programa que calcula as estimativas modais de $\boldsymbol{\theta}$. Modelo de Poisson 


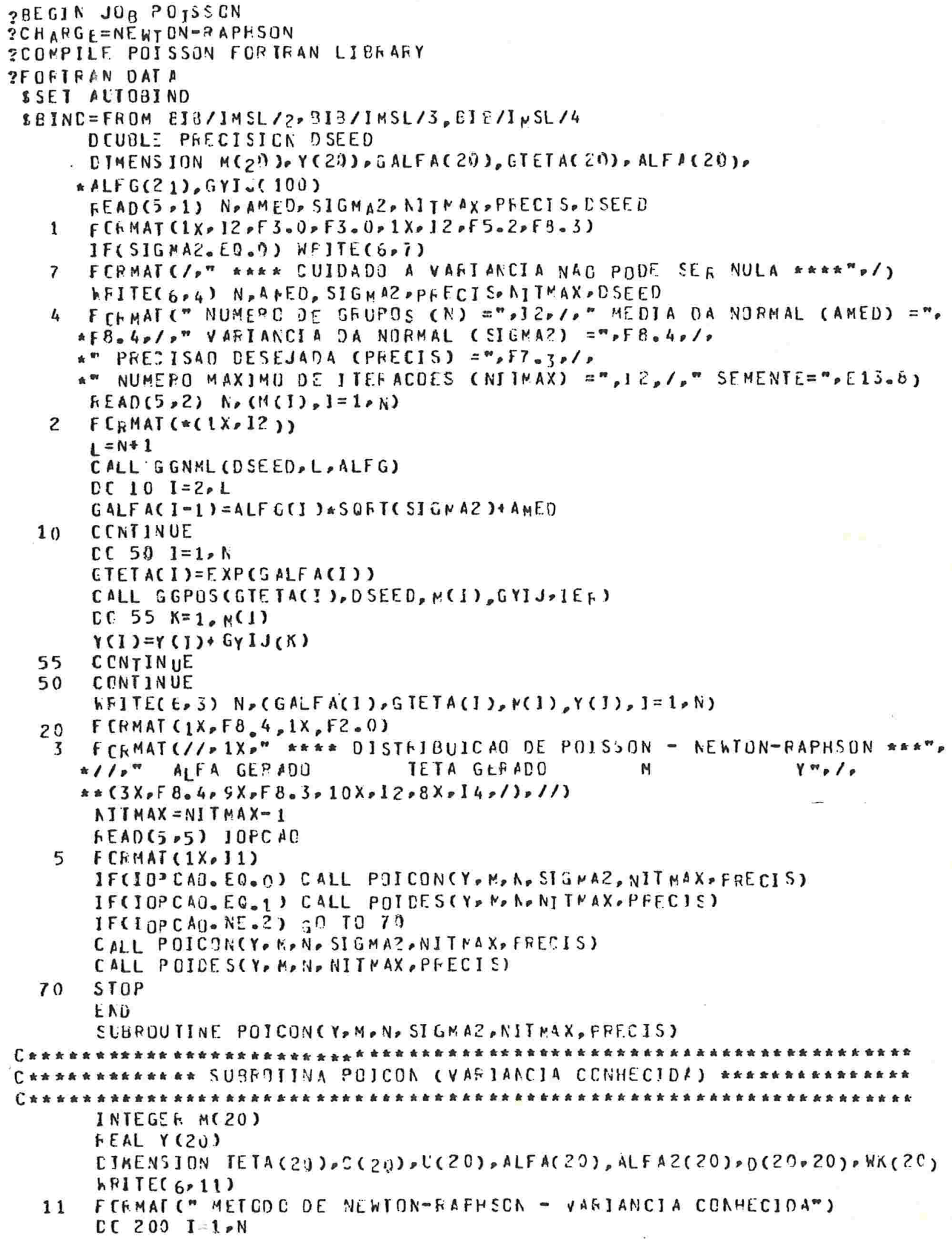


IEI $A(I)=Y(1) / F L 0 A T(M(1))$

I $F\left(T E T A(I) \cdot E C_{0} C_{0}\right)$ TETA(I) $=C_{0}$ C C 1

$A L F A(I)=A L D C(T E T A(J))$

$A L F A S O=A L F A S C+A L F A(I)$

ZUD CCNTJNUE

$210 \quad A L F A S=A L F A S O / F L D A T(N)$

CC $220 \quad I=1, N$

ATETA $=E X P(A L F A(I))$

$C(1)=Y(J)-F L O A T(M(J))=A T E T A-(A L F A(J)-A L F A S B) / \subseteq J C P A Z$

$l(1)=(F L J A T(\mu(1)) * A T E T A * 1 / S] C M A 2) * *(-1)$

(SOMA $=U S O M A+U(I)$

220 CTNTINUE

ALF ASO $=0$.

$U S O M A=U S D M A / F L C A T(N)$

CC $230 \quad I=1, N$

Dr $240 J=I, N$

$[(1, J)=U(J) * U(J) /(F L O A T(N) *(S] G N A Z-U S O N A))$

$C(j, 1)=D(I, j)$

240 CCNTINUE

230 CCNTINUE

I FRE. $C=C$

ISOMA $=0$.

C. $250 \quad]=1, N$

PPOD $=0$.

$C[260 \quad]=1, N$

$F P O D=P R O D+D(J, J) * C(J)$

260 CCNTINUF.

$A L F A 2(I)=A L F A(I)+P R O D$

$A L F A S O=A L F A S O+A L F$ Q 2(1)

$A L F=A_{L} F A(I)$

$1 F(A L F . E Q .0) \quad A L F=0.001$

$A \cup X=A B S((A L F A Z(I)-A L F A(I)) / A L F)$

IF (AUX.GT.FFEC IS) IPFEC =1

2SO CCNT]NUE

AITEF $=N$ I T E. K +1

I.F(NITEK.LE.AITMAX.OH.IPFEC,EG.0) GO TO 270

hFITE 6.15$)$

15 CFMAT $(/, 1 X$ " NAT CDNYERGIU ATE O NUMERO MAXINO DE ITERACOES")

6 C TO 290

270 IF(IPFEC.E.0.0.) GD TO 290

C[: 280$]=1, \mathrm{~N}$

$A L F A(I)=A L F A 2(I)$

280 CENTINUE

G T TO 210

290 CC $300 \quad I=1, N$

TFTA I I $)=E X F(A L F A 2(I))$

300 CCNTINUE

hFITE(E, 17) NJTEF,N, $(A L F A 2(1), \operatorname{TETA}(1), C(1), 1=1, N)$

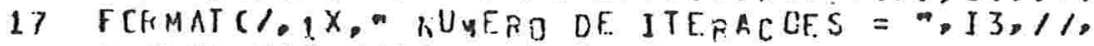

* ALFA ESTIMADE TETA ESTIMADO DEHIVADA (VETCF C)",

$*(3 X, F E, 4, E X, F E, 3,6 X, E 15,3,1))$

WFI TE $(6.550)$

550 FCFMAT $(/, 1 X, "$ NATFIT C IAVEFSA DA ULTINA ITEK CAC",//) or $5 \mathrm{El}) \quad 1=1, \mathrm{~N}$

$h F 1 T E(6,18) N,(D(3, J), J=1, N)$

18 FCFMAT $(*(1 X, E 9.3), 1)$

500 CCNTINUE

$D 1=0$.

I $20 \mathrm{~B}=4$ 
CALL L INV ZF(D. ALFA,1 JOH, N, 20, D 1, U2, hK, JEG)

$\mathrm{CET}=\mathrm{D} 1 * 2.0 * \mathrm{D} 2$

$A L F A S O=A L F A S O / F L U A T(N)$

WFIIEC 6,19$)$ DE I

19

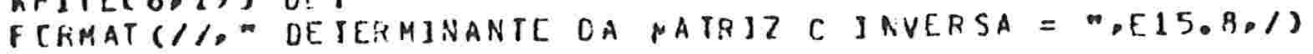

FETURY

END

SUBFOJTINE POIDES ( $Y_{P} M_{D} N, N I T N A X$ PFECJ

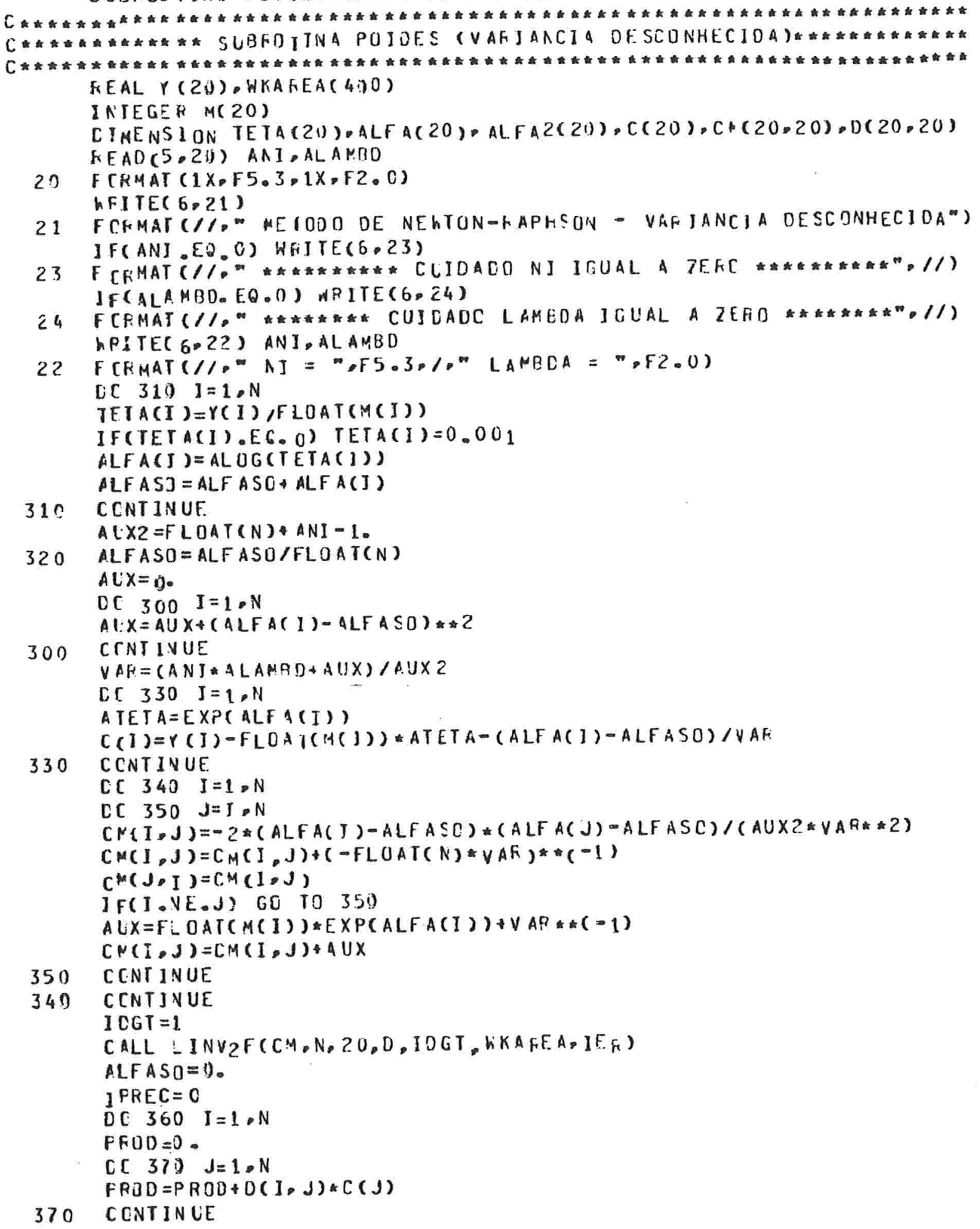




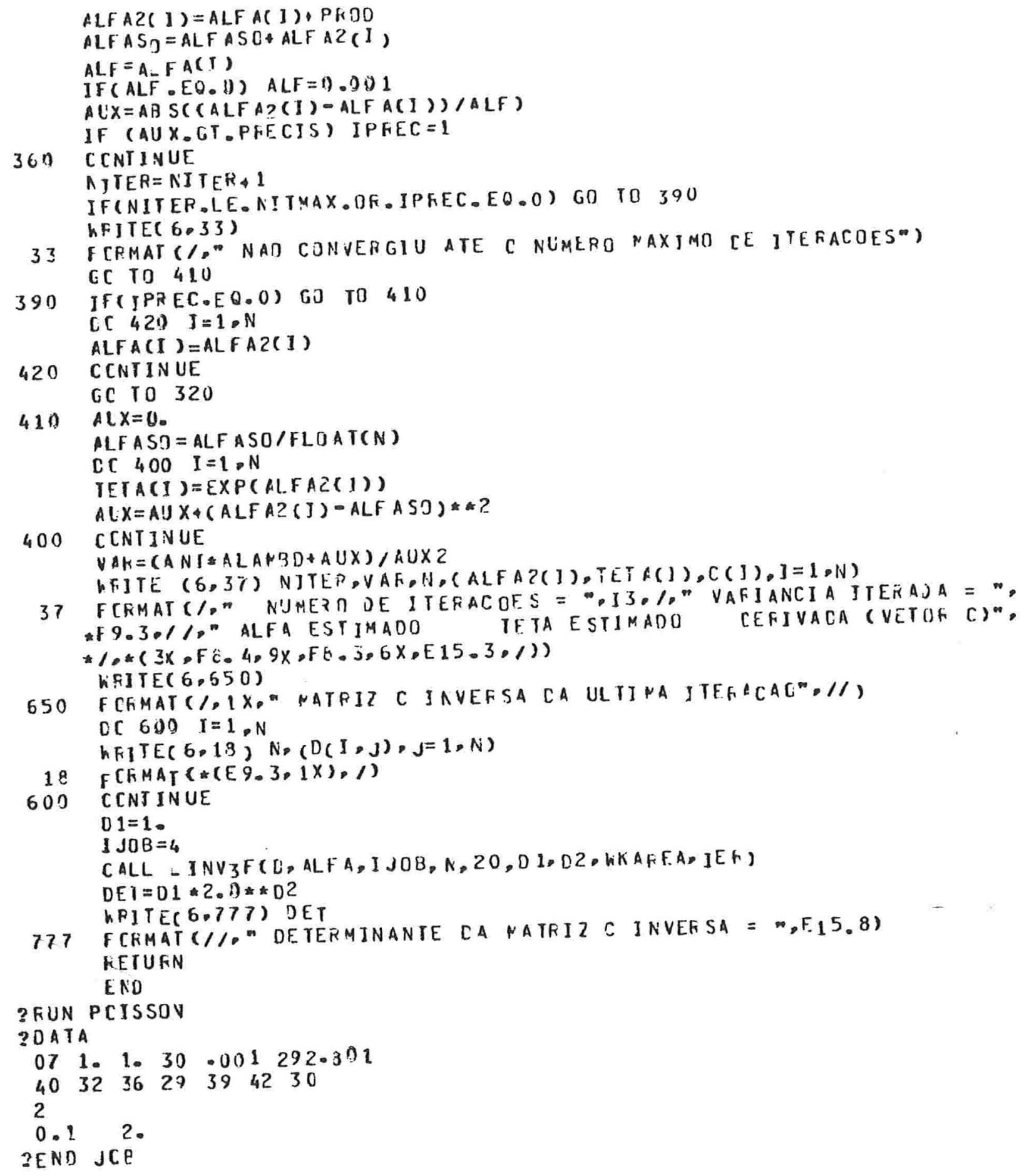


NUMERO DE GFUPOS $(N)=7$

MEDIA DA NORMAL (AME)) $=1.0000$

VARIANCIA DA NDRMAL (SIGMAZ) $=1.0000$

PRECISAO DESEJADA (PIECIS) = 0.NOI

NUMERO MAXIMO DE I TERACOES (NITMAX) $=30$

SEMENTE $=.29230100 E+03$

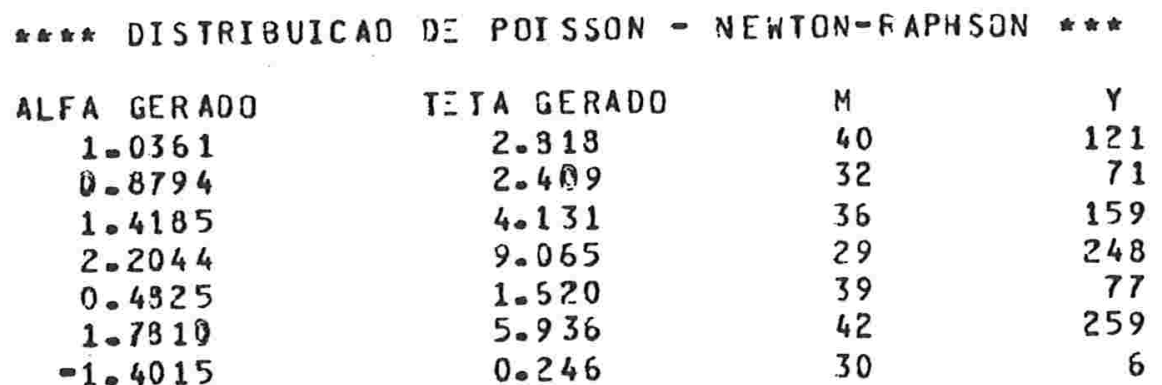

METODO DE NEWTON-RAPHSON - VARIANCIA CONHEEIJA

NUHERO DE ITERACDES $=3$

$\begin{array}{ccc}\text { ALFA ESTIMADO } & \text { TETA ESTIMADO } & \text { DERIVADA (VETDR C) } \\ 1.1057 & 3.021 & -.204 E=06 \\ 0.7992 & 2.224 & -.353 E-06 \\ 1.4321 & 4.402 & -.189 E-06 \\ 2.1414 & 8.511 & -.193 E-06 \\ 0.6839 & 1.982 & -.355 E-06 \\ 1.8159 & 6.146 & -.135 E=06 \\ -1.2906 & 0.275 & \end{array}$

MATRIZ C INUERSA DA ULIIMA ITERACAO

$$
\begin{array}{lllllll}
.822 E-02 & .166 E-04 & .752 E-05 & .484 E-05 & .153 E-04 & .463 E-05 & .129 E-03 \\
.166 E-04 & .139 E-01 & .127 E-04 & .317 E-05 & .259 E-04 & .781 E-05 & .219 E-03 \\
.752 E-05 & .127 E-04 & .628 E-02 & .370 E-05 & .117 E-04 & .354 E-35 & .989 E-34 \\
.484 E-05 & .317 E-05 & .370 E-05 & .404 E-02 & .753 E-05 & .228 E-05 & .637 E-04 \\
.153 E-144 & .259 E-04 & .117 E-04 & .753 E-05 & .123 E-01 & .720 E-035 & .202 E-03 \\
.463 E-05 & .731 E-05 & .354 E-05 & .228 E-05 & .720 E-05 & .386 E-02 & .609 E-04 \\
.129 E-03 & .219 E-03 & .989 E-04 & .537 E-04 & .202 E-03 & .509 E-04 & .110 E-00
\end{array}
$$

DEIERMINANTE DA MATRIZ C INVERSA $=.15665020 E-13$ 
METDDD DE NEWTON-RAPHSON - VARIANCIA DESCONHECIDA

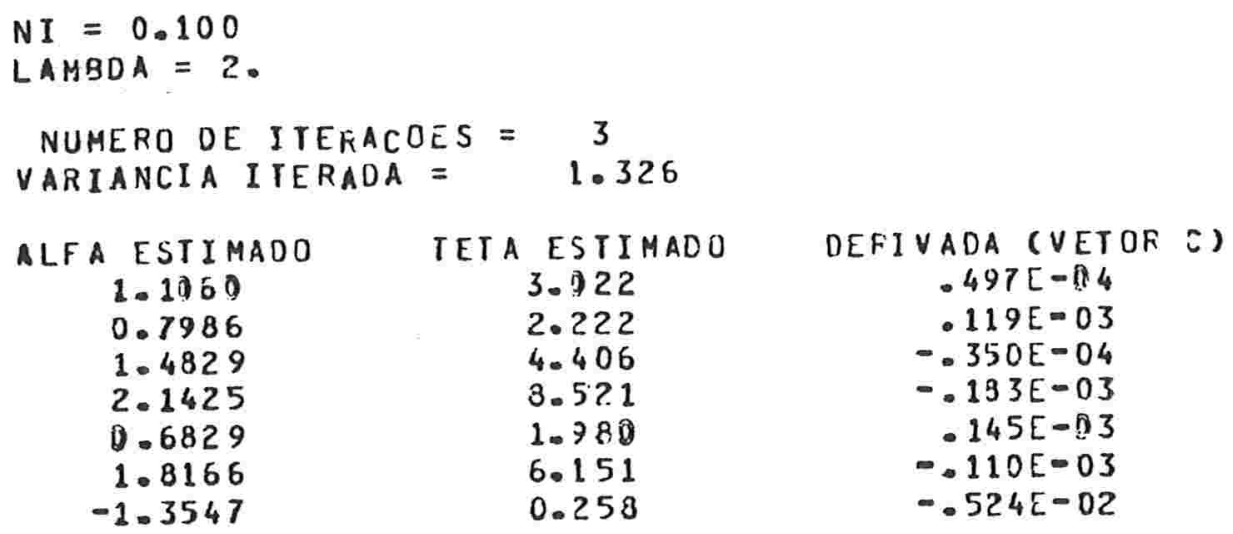

MATRIZ C INVERSA DA ULTIMA ITERACAO

$\begin{array}{lllllll}.823 E-02 & .120 E-04 & .534 E-05 & .455 E-05 & .107 E-04 & .418 E-05 & .470 E-04 \\ .120 E-04 & -139 E-01 & .788 E-05 & .352 E-05 & .217 E-04 & .414 E-05 & .329 E-03 \\ .634 E-05 & .783 E-05 & .528 E-02 & .590 E-05 & .627 E-05 & .430 E-05-.102 E-03 \\ .465 E-05 & .362 E-05 & .590 E-05 & .404 E-02 & .135 E-05 & .491 E-05 & -.221 E-03 \\ .107 E-04 & .217 E-04 & .627 E-05 & .136 E-05 & .128 E-01 & .231 E-05 & .390 E-03 \\ .418 E-05 & .414 E-05 & .480 E-05 & .491 E-05 & .281 E-05 & .386 E-02-.138 E-03 \\ .470 E-04 & .329 E-03-.102 E-03-.221 E-03 & .390 E-03-.138 E-03 & .135 E+00\end{array}$

DETERMINANTE DA MATRIZ C INVERSA $=.19523320 E=13$ 
APÊNDICE 5 - Saída e listagem do programa que calcula as estimativas de $\theta$ baseadas no método de Laplace. Modelo de Poisson 
3BEG]N JUB POISSUN

?CHAFGE $=L A P L A C E$

?COMPILE PUISSON FOR TRAN LJBRAFY

ZFOGTFAA DATA

SSET AUTOBIND

$\$ B$ ! NC = FRDM EIB/IMSL/2, D]B/IMSL/3,EIE/I MSL/4

CIMENS ION $M\left(2^{\prime \prime}\right), Y(29)$

hFITE( 6,10$)$

10 FCFMAT ${ }^{*}$ **** DISTRIBUICAO DE PCISSON-METODO DE LAPLACE *****, * I )

FEAD(5,1) N, SIGNA2,N1TMAX,PFECIS

1 FCRMAT $\left(1 X_{0} 12,\left(X_{0} F 3,0,1 X_{0} 13,(X, F 5,2)\right.\right.$

IF(SIGNAZ.EQ.1)) WFITE $(6.7)$

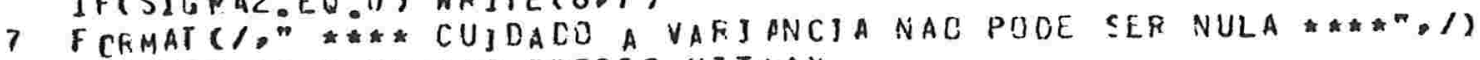

WFITE 6,4$)$ N.SIGMAZ,PRECIS,NITFAX

4 FCKMAT ( $"$ NUMERD DF. GRUPOS $(N)=m, 12,1$.

" "VAKI ANCIA DA NJFMAL (SIGMAZ) $=", F B .4 \% 1$,

" PRE:ISAD DESEJADA (PHECJS) = " F?.3,1,

* NUMEFIO MAXJMG DE ITEFACOES (NITMAX) =", I2, 1)

$\operatorname{KEAD}(5,1)(M(I), 1=1, N)$

KEAD (5, / ) $(Y(T), 1=1, N)$

hFITE( 6,25$) \quad N=(M(I), Y(1), I=1, N)$

25 FCFMAT " TAMANHO DA AMOSTFA

$Y=, 1, *(8 x, 12,17 x, 14,1))$

A I TMAX $=$ NI T Y $A X-1$

FEAD $(5,5)$ IOPCA?

5 F(KMAT $(1 X, 11)$

IF(IOPCAU.EO.O) CALL LAPCCUCY,MON,S1GMAE, NITMAXPPFECIS)

JF(JOPCAO.EQ.1) CALL LAPCOEC $Y, M, A, N J T M A X, P R E C 1 S)$

IF (IDP $C A O \cdot N E .2)$ GD TO 70

CALL I APUCOC $Y$ P M N. SIGMAZ,NITHAX, FFECIS)

CALL - APODE $(Y, M, N, N I T M A X, P F E C I S)$

70 SIOP

END

SUBFOJ IUNE LAPOCO(Y,M,N口SIGMAZ,NITMAX,PFECIS)

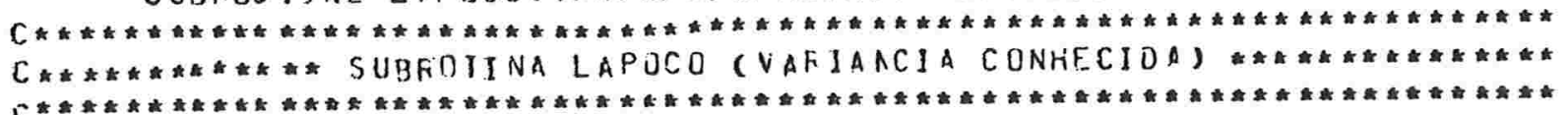

INTEGER $M(20)$

FEAL $Y(20)$

CIMENSION TETA(20), C (20), U(20), ALFA(20), ALFA2(20),D $(20,20), W K(20)$,

* ALFCO( 20)

WFITE $(6,11)$

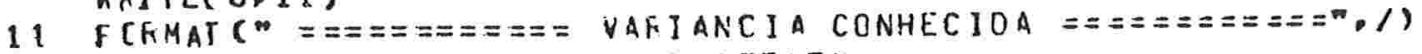

HEAD(j,/) (ALFCO(I),I $=1, A), D E T N E K$

DC $2031=1, N$

ALFEST $=$ ALFEST ALFCO(I)

203 CENTIN UE

$A L F E S T=A L E E S T / F L O A T(N)$

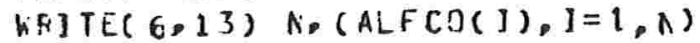

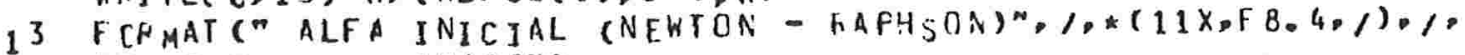

* $33 x_{0}$ " TETA ESTJMADO")

C! $5000 \quad L=1, N^{\circ}$

$A L F \& S]=0$ 。

$U \subseteq O M A=0$.

A I J EF $=0$

C. $200 \quad]=1, N$

$A L F A(I)=A L F C C(I)$ 


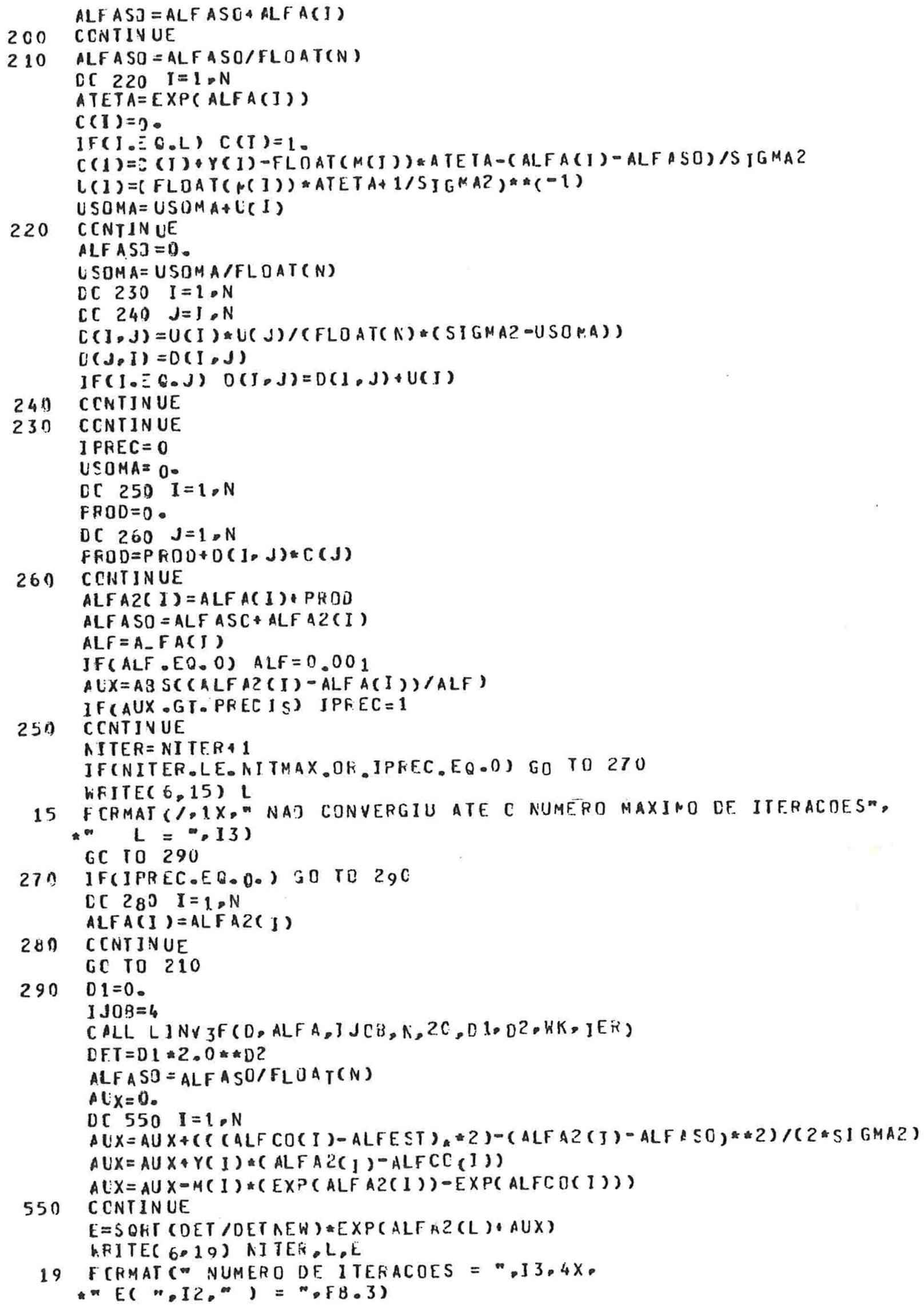




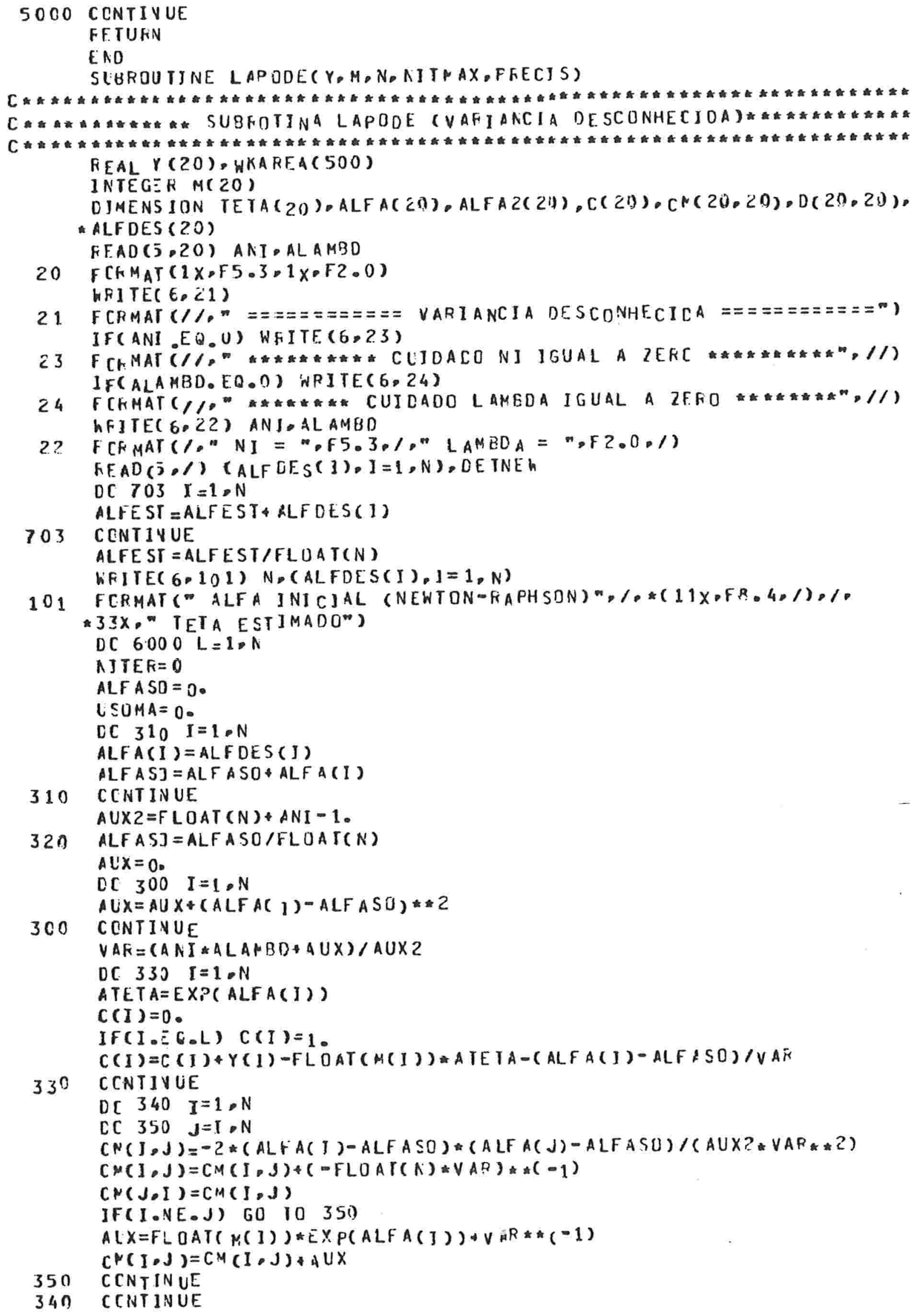


I $\mathrm{CGT}=1$

CALL LINVZF(CM,N, 20,D, IDGT, WKAFEA,IEN)

$A L F A S J=0$.

I PREC $=0$

DC $360 \quad \mathrm{I}=1 . \mathrm{N}$

$\mathrm{FPOD}=0$.

DC $370 \quad J=1 . N$

$F F Q D=P R O O * D(1 \cdot J) \approx C(J)$

370 CCNTINUE

ALFAZC I $=A L F A(I)+P R D D$

$A L F A S O=A L F A S O+A L F A 2(1)$

$A L F=A-F A(I)$

IF (ALF.EO.O) ALF $=0.001$

$A \cup X=A B S(C(A L F A Z(I)-A L F A(I)) / A L F)$

IF (AUX。GT.PFECIS) IPFEC $=1$

$36 \cap$ CENTINUE

AIJEF $=$ NITEK+ 1

IF(NITER.LE. NITHAX.OF.IPFEC.EQ.O) GO TO 390 hPITE $(6.33)$

33 F CRMAT (/, NAD CDNVEFGIU ATE O NLMERO NAXIMO [E ITEFACDES")

GC TO 410

390 IF(IPFEC.EQ.0) GO TO 410

C [ 420 ] $=1, N$

$A L F A(I)=A L F A 2(I)$

420 CENTINUE

C.C TO 324

$410 \quad A L X=0$.

ALFASO = ALFASO/FLOAT(N)

CC $400 \quad I=1 . N$

$A L X=A U X+(A L F A 2(J)-A L F A S O) * 2$

${ }_{4} 00$ CENTINUE

VAF $=(A N I=A L A+3 D+A \cup X) / A U X 2$

$D 1=1$.

$1 \mathrm{JOB}=4$

CALL LINV3F (C, ALFA, IJOB, N, 20, D 1, D2, KKAFEA, IF, F)

CET $=D 1 \approx 2.11 * 02$

$A L X=A V I \notin A L A M B D$

$A \cup X I=A N I * A L A \cap B D$

$\operatorname{LXX} 3=0$.

CC $600, I=1, \mathrm{~N}$

$A L X=A \cup X+(A L F A 2(I)-A L F A S O) * * 2$

$A(X)=A \cup X_{1}+(A L F U E S(I)-A L F E S T)=2$

$A L X 3=A \cup X 3+Y(I) *(A L F A 2(1)-A L F D E S(3))$

$A(X 3=A \cup X 3=M(I) *(Z X P(A L F A Z(I))-E X F(A L F D E S(I)))$

600 CENTINUE

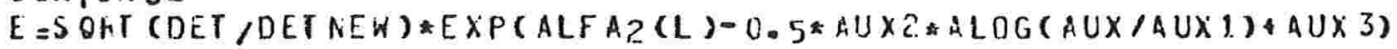

KRITE( 6.777$)$ NITER,L,E

777 FCFMAT $(*$ NUMERD DF ITEFACOES $=*, 13,4 X$,

* $E(m, I 2, \cdots)=", F 8.3)$

6000 C[NT INUE

FETUFN

END

?FUN PCISSON

?DATA

C7 1. $30 \quad 0.001$

$40,32,36,29.39,42,30$,

121.71 .159 .248 .77 .259 .6 .

2

$1.1057,0.7992,1.4821 .2 .1414 .0 .6835 .1 .8159,-1.2906$.

$0.15665020 E-13$

0.1002.

$1.106 C \cdot 0.7986 \cdot 1.4829 \cdot 2 \cdot 1425 \cdot 0.6829 \cdot 1.8166 .-1.3547$.

$0.19528320=-13$,

?END JCB 


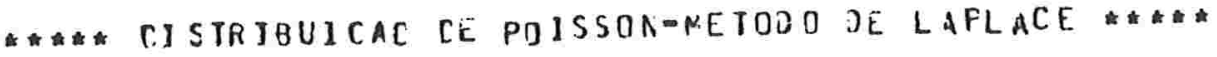

NUMEKO DE GPUPCS $(A)=7$

VAFIANCIA DA NOFMAL (SIGFAC) $=1.0000$

PRECISAO DESEJADA (PRECIS) = C.CCL

NUREFO MAXIMO DF. I JEFACOES (NITMAX) $=30$

TAMANHC. DA AMOSTRA
$4 C$
32
36
29
35
42
30
$========$ VARIAA
ALFA INICIAL CAFST
1.1057
0.7992
1.4821
2.1414
0.6839
1.8159
-1.2906

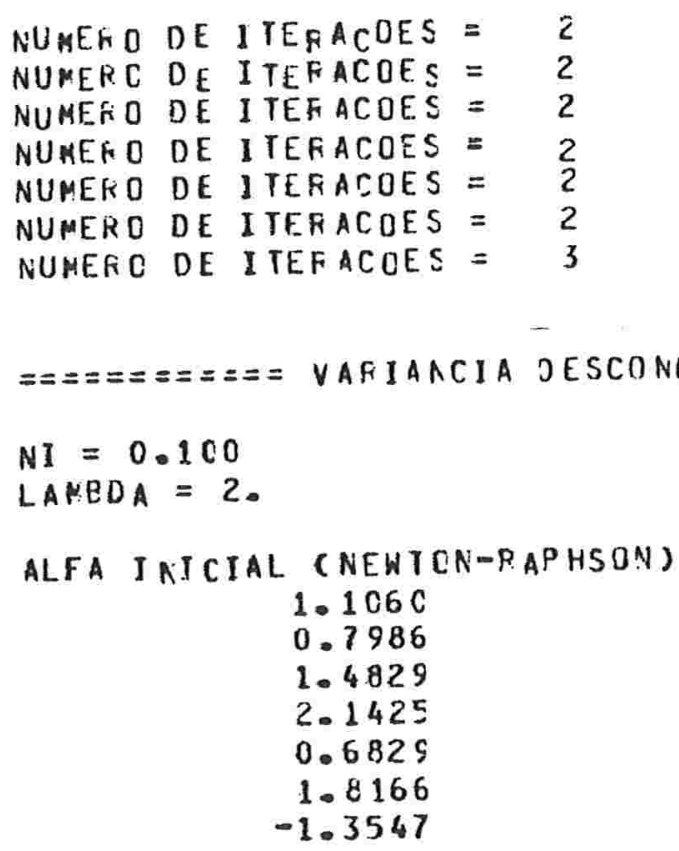

NUAERC DE. ITERACOES TETA ESTIMADO

$E(1)=3.023$

$E(2)=2.225$

$E(3)=4.404$

$E(4)=8.515$

$E(5)=1.982$

$E(0)=6.149$

E( $r)=0.277$

NUPEFO DE ITEF $A C O E S$ NUREFC DE ITEFACOES = NURER DE ITEFACOES = NUMERO DE I TEFACOES = NUMEFO DE I TEFACOES = NUAER̃O DE ITEFACOES =

2
2
2
2
2
2
3

\begin{tabular}{|c|c|c|c|}
\hline \multicolumn{3}{|c|}{ TE' A } & $E S T]^{N}{ }^{N} D O$ \\
\hline EC & 1 & ) & $=\quad 3.023$ \\
\hline & 2 & ) & 2.223 \\
\hline EC & 3 & ) & $4.4 \mathrm{C7}$ \\
\hline$E C$ & 4 & ) & 8.524 \\
\hline Er & 5 & ) & 1.980 \\
\hline$E$ & ن & ) & 6.154 \\
\hline E & 7 & ) & 0.257 \\
\hline
\end{tabular}


APÊNDICE 6 - Saída e listagem do programa que calcula as estimativas de $\theta$. Modelo normal 


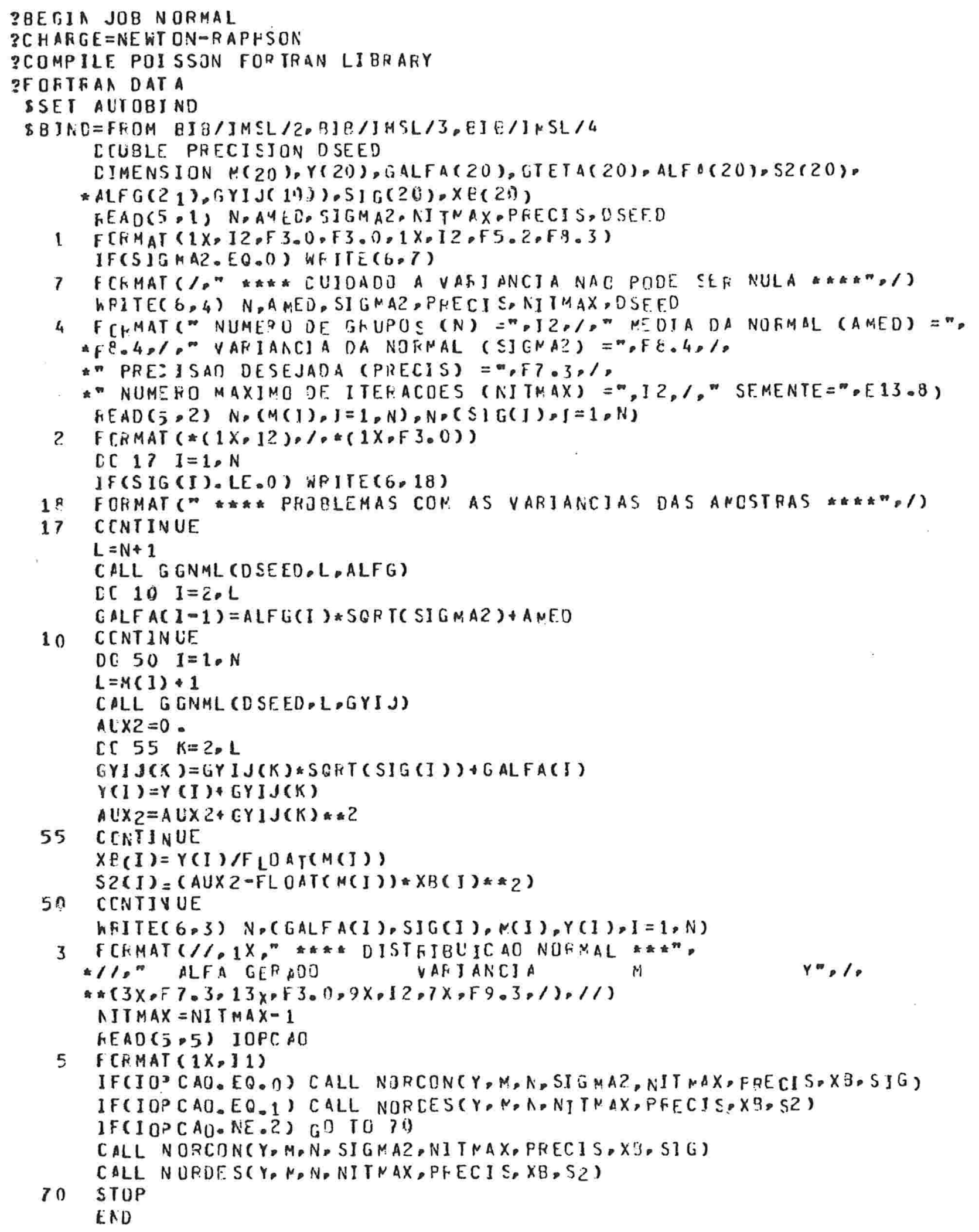


SLBROUTINE. NDQCON $Y, M, N, S I G N A 2, N I T N A X, F F E C I S, X E, S T G)$

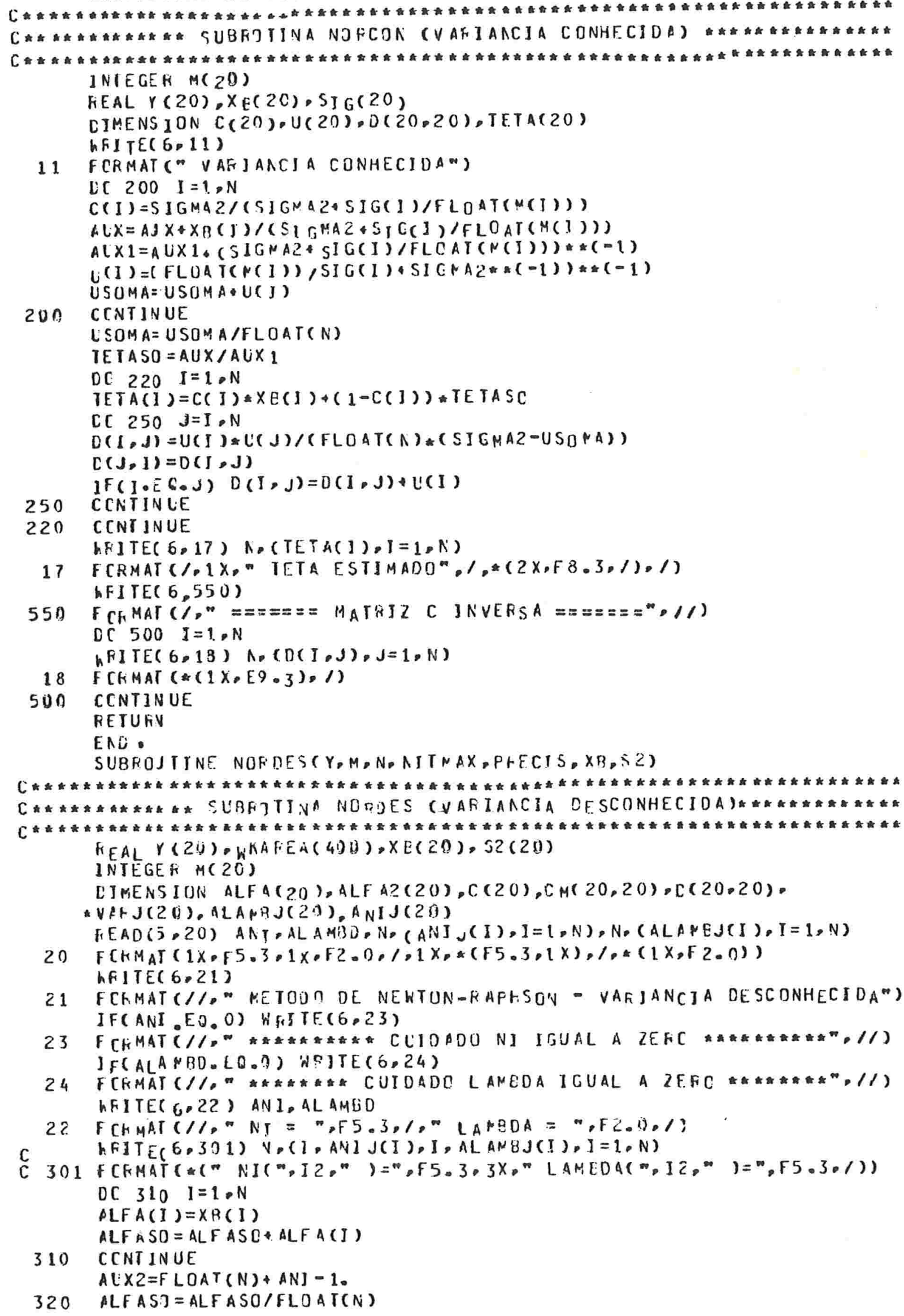




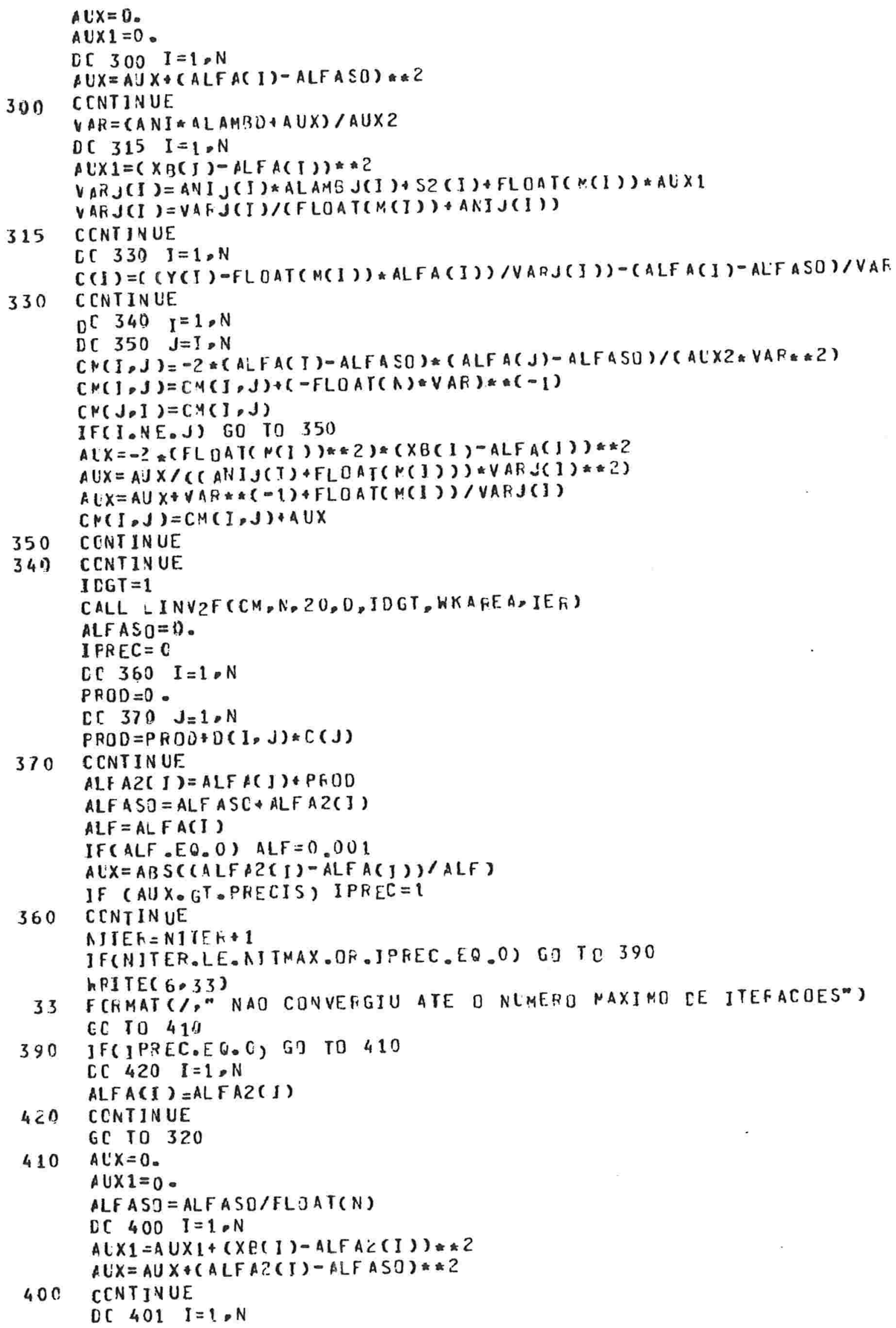




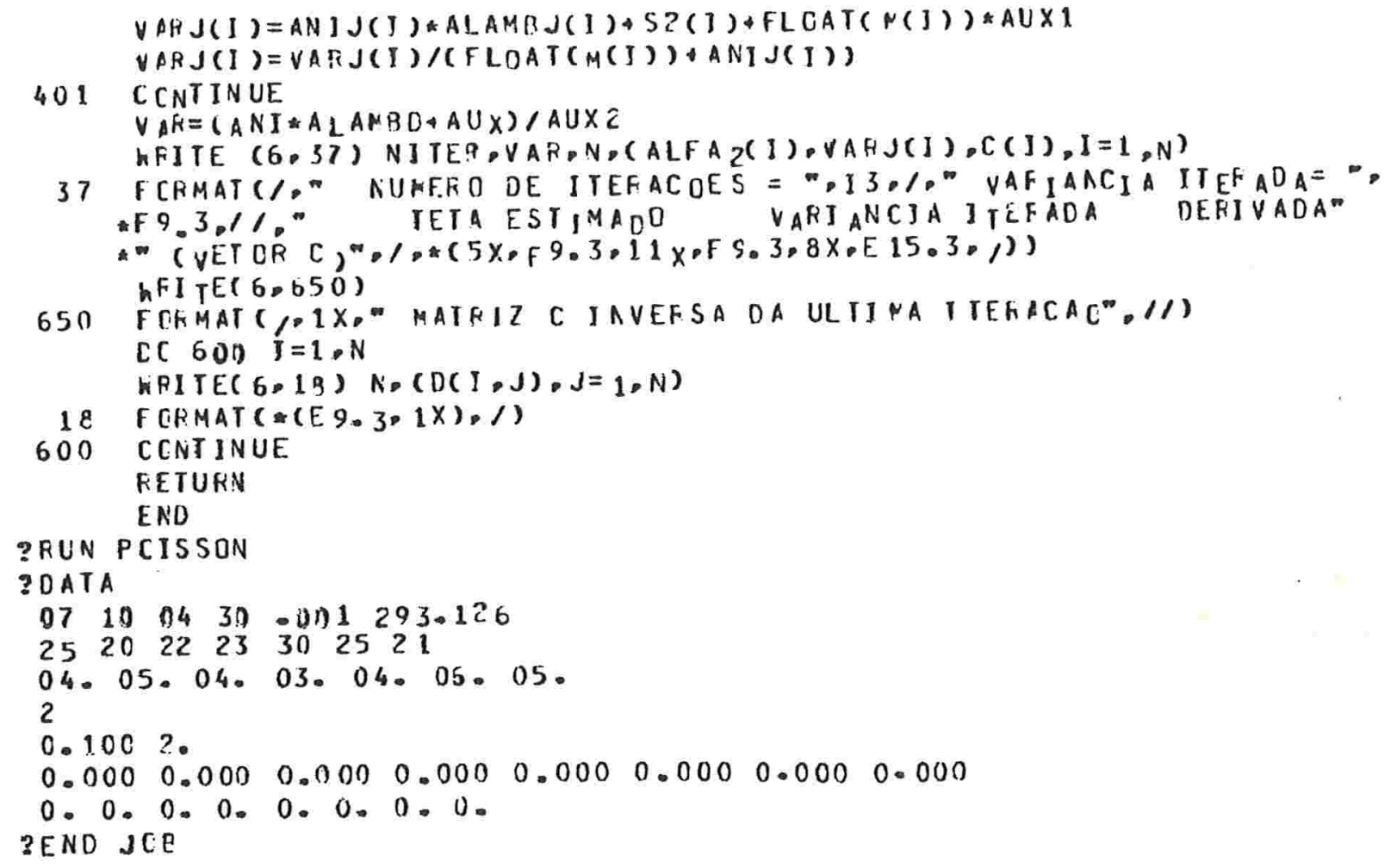


NUMERD DE GRUPOS (N) $=7$

MEDIA DA NOFMAL (AMEJ) $=10.1900$

VARIANCIA DA NORMAL (SIGMAZ) $=4.0000$

PRECISAO DESEJADA (PFECIS) $=0.001$

NUMERO MAXIMO DE ITRAACOES (NITMAX) $=30$

SEMENTE $=.29312600 E+03$

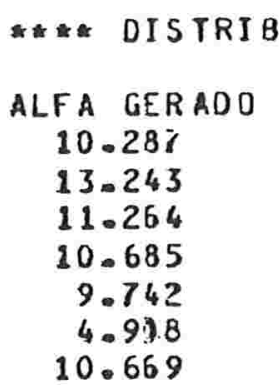

VARIANCIA
40
5.
$40^{\circ}$
$30^{\circ}$
40
6.
5.

$M$
25
20
22
23
30
25
21

$r$

266.965

268.398

254.381

254.956

298.584

111.604

224.547

VARIANCIA CDNHECIDA

$$
\begin{aligned}
& \text { TETA ESIIMADO } \\
& 10.653 \\
& 13.230 \\
& 11.507 \\
& 11.059 \\
& 9.965 \\
& 4.793 \\
& 10.659
\end{aligned}
$$

$======+$ MATRIZC INVERSA $=======$

$$
\begin{aligned}
& .155 E+00 \quad .135 E-02 \quad .100 E-02 \quad .727 E-03 \quad .743 E-03 \quad .130 E-02 \quad .129 E-02 \\
& -135 E-02 \quad-231 E+00 \quad-153 E-02 \quad-111 E-n 2 \quad-114 E-02 \quad .199 E-02 \quad .198 E-02 \\
& .100 E-02 \quad .153 E-32 \quad .175 E+00 \quad-822 E-83 \quad .843 E-03 \quad-147 E-02 \quad .146 E-02 \\
& -727 E-03 \quad-111 E-02 \quad .822 E-03 \quad-127 E+00 \quad-610 E-03 \quad .107 E-02 \quad .108 E-02 \\
& .743 E-33 \cdot 114 E-12 \cdot .849 E-53 \quad-619 E-133 \quad .132 E+00 \quad .199 E-92 \quad-198 E-92 \\
& .130 E-02 \quad .199 E-02 \text {. 147E-02 -107E-n2 .109E-n2 .228E-nG . } 190 \mathrm{E}-132 \\
& .129 E-02 \quad .198 E-02 \quad .146 E-02 \quad .106 E-02 \quad .109 E-02 \quad .190 E-02 \quad .227 E+00
\end{aligned}
$$


METODO DE NEWTON-RAPHSON - VARIANCIA DESCONHECIDA

$N I=0.100$

$\angle A M B D A=2$.

NUMERO DE ITERACOES $=\frac{2}{7.228}$
VARIANCIA ITERADA $=$

$\begin{array}{ccc}\text { TETA ESTINADO } & \text { VA.SIANCIA ITERADA } & \text { DERIVADA (VETOR C) } \\ 10.671 & 3.347 & -.237 E-04 \\ 13.349 & 3.168 & -.993 \mathrm{E}-03 \\ 11.532 & 3.911 & -.153 E-03 \\ 11.073 & 2.473 & -.115 \mathrm{E}-03 \\ 9.961 & 3.364 & .191 \mathrm{E}-03 \\ 4.635 & 5.481 & .400 \mathrm{E}-02 \\ 10.677 & 5.735 & .291 \mathrm{E}-04\end{array}$

MATRIZ C INVERSA DA ULTIMA ITERACAO

\begin{tabular}{|c|c|c|c|c|c|c|}
\hline $13 O E=O D$ & $.566 E-03$ & $26 \Xi-03$ & $03 E-03$ & $.275 E-03$ & $.153 E-03$ & $73+73$ \\
\hline $5655-013$ & & & & & $=.313 E=02$. & $M, E \Rightarrow 0$ \\
\hline 526 & $1215-0$ ? & $.173 E \cdot 00$ & $.433 E-0$ & & & \\
\hline 3) $3 E-03$ & $.586 E-j 3$ & $.483 E-33$ & $.205 E+[0$ & $.212 E-03$ & $-.215 E-03$ & \\
\hline 28 & .232 & $.331 E-03$ & $.212 E-03$ & $.110 E+00$ & $.753 E-03$ & .55 \\
\hline-03 & $313 E-02$ & $-.989 E-03$ & $-\angle Z J L-U$ & .75 & & \\
\hline & & $1065-0$ & $610 E=03$ & $.554[-03$ & $.274 E-03$ & $.263 E \cdot 0$ \\
\hline
\end{tabular}


APENDICE 7 - Amostragem em dois estágios Populacões e amostras simuladas. Modelo binomial 

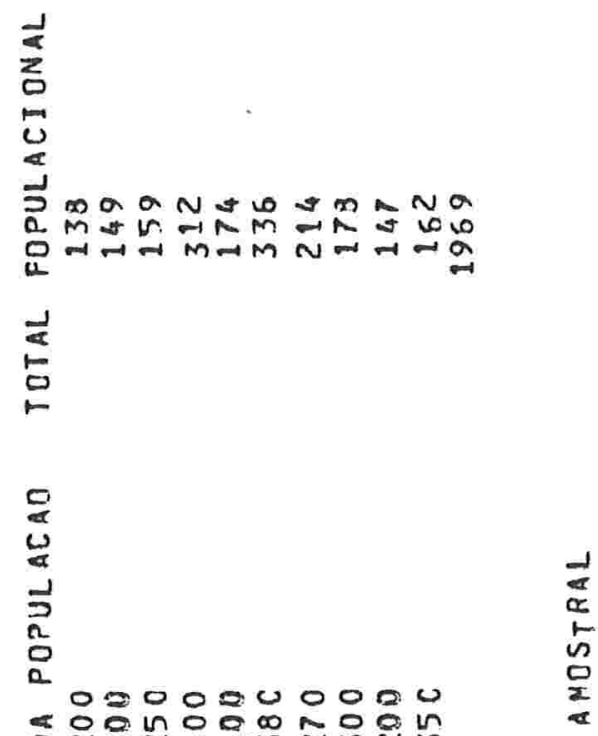

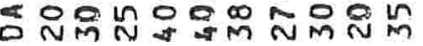

$\frac{0}{2}$

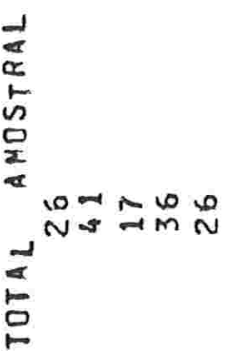

$\circ$
0
0
$\circ$
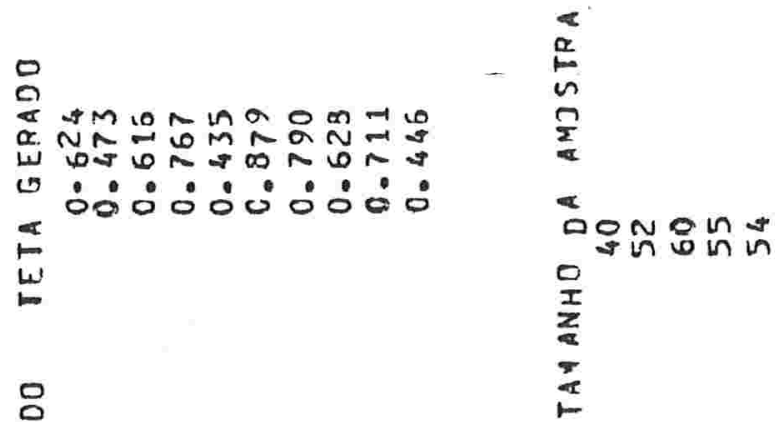

造

유" 는

n

员

wamam $20-6$

טOOR

แก

v) $\begin{array}{lll}\alpha & \alpha & 0\end{array}$

멈요

a o 2 in

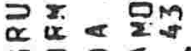

6 인?

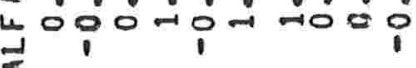

山 w"

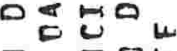

민

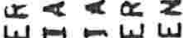

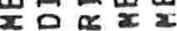

Dw
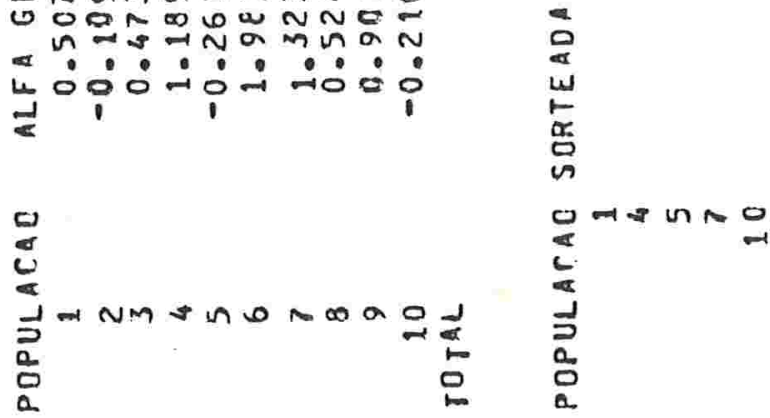
APÊNDICE 8 - Amostragem em dois estágios Populações e amostras simuladas. Modelo de Poisson 


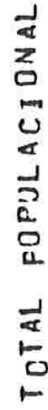

0
0
0
$a$
$a$
$a$
0
0
0

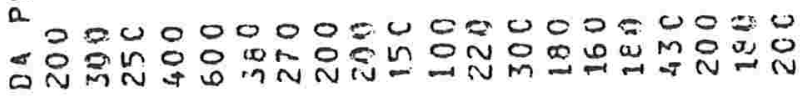

$\frac{0}{2}$

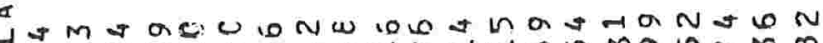
$\vec{a} \approx$ ज

$\frac{1}{5}$

2.

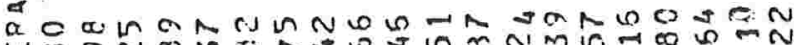

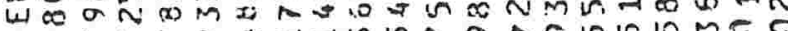

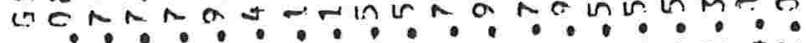

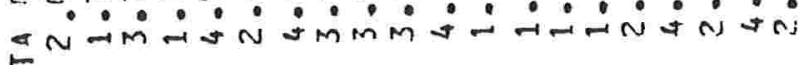

Li

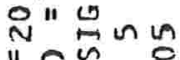

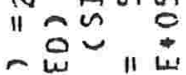

뭉

$\leq x-10$

is $x$

v. 4 L

므요

a 2 织

$\pi$
4 $\frac{0}{2}$

जo 0 a?

$\omega^{2}=\omega "$

○匹ら口ル

0 z

的的岕

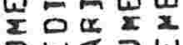

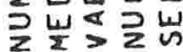

구뇨

aUn $N$

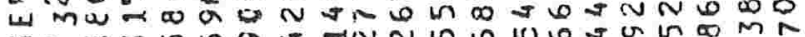

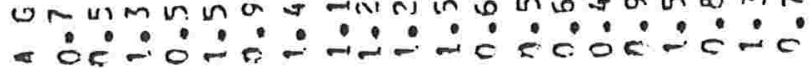

$\frac{4}{\frac{1}{\sigma}}$

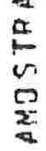

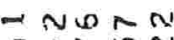

$\sin _{\pi} \sin n$

$\underset{b}{\infty}$
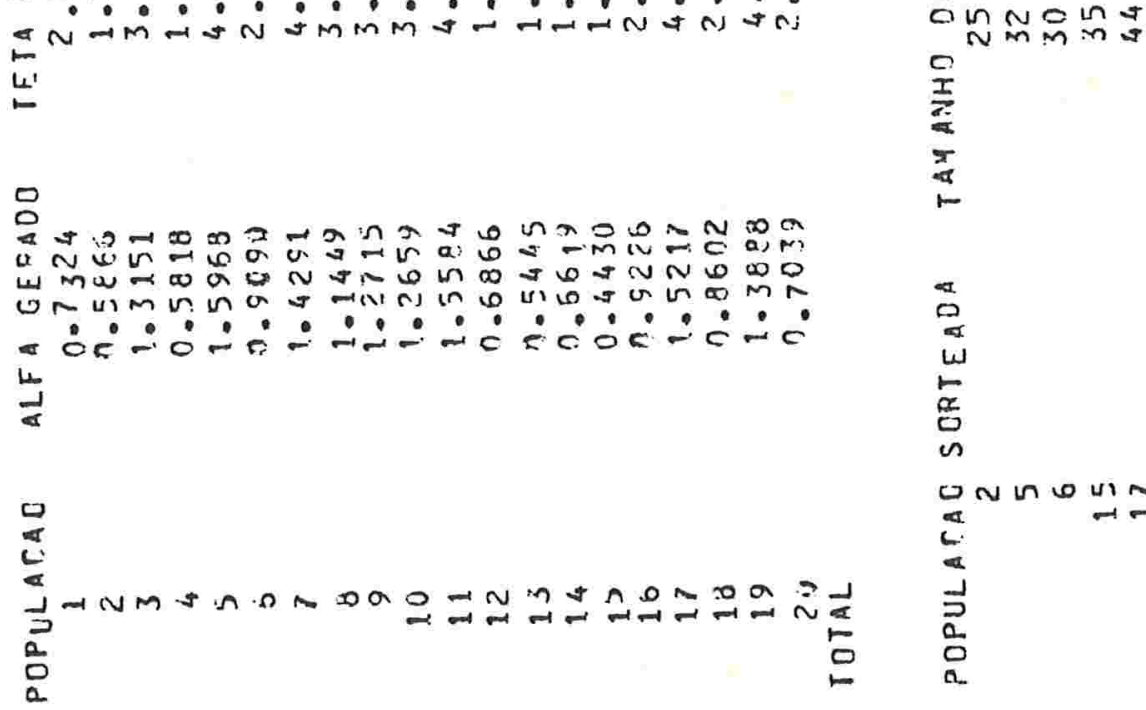

a

on $\cos 2$

뭄 
APÊNDICE 9 - Amostragem em dois estágios Populações e amostras simuladas. Modelo normal 

1
0
2
0
0
$\vdots$
$\vdots$
0
0
0
0
0
0
0
0
0
0
0
0

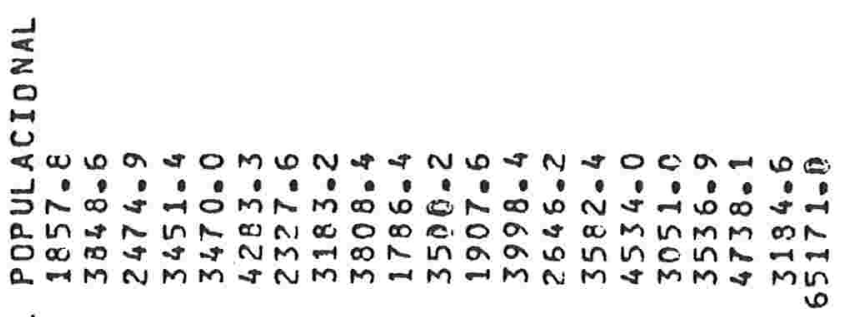

㝴

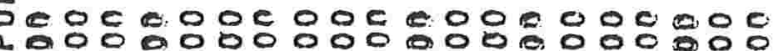

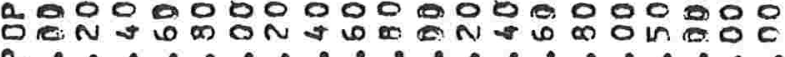

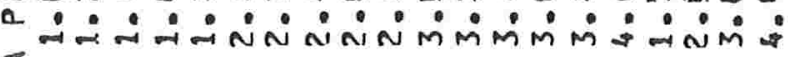

음
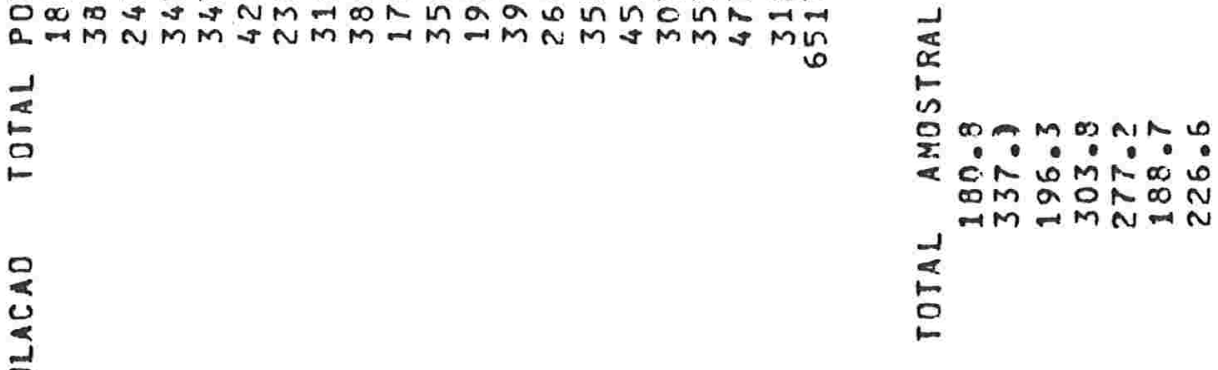

믕을 은 ONMNMMMNNONNMNON N

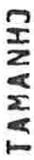

c. $11 \stackrel{\sum^{2}}{i=1}$

ก

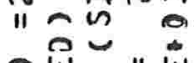

z㘳, 11 岁

a 420

กา.

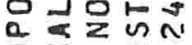

$\underset{x}{x} \sum \sum_{0}^{\infty}$

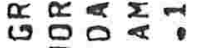

อ

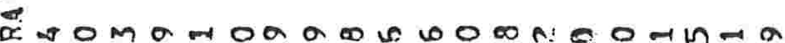

山ึ่

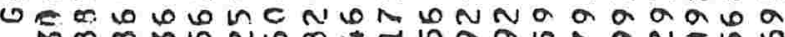

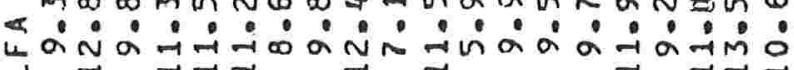

$<$

w

임네

문

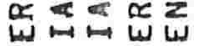

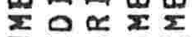

崖岁少
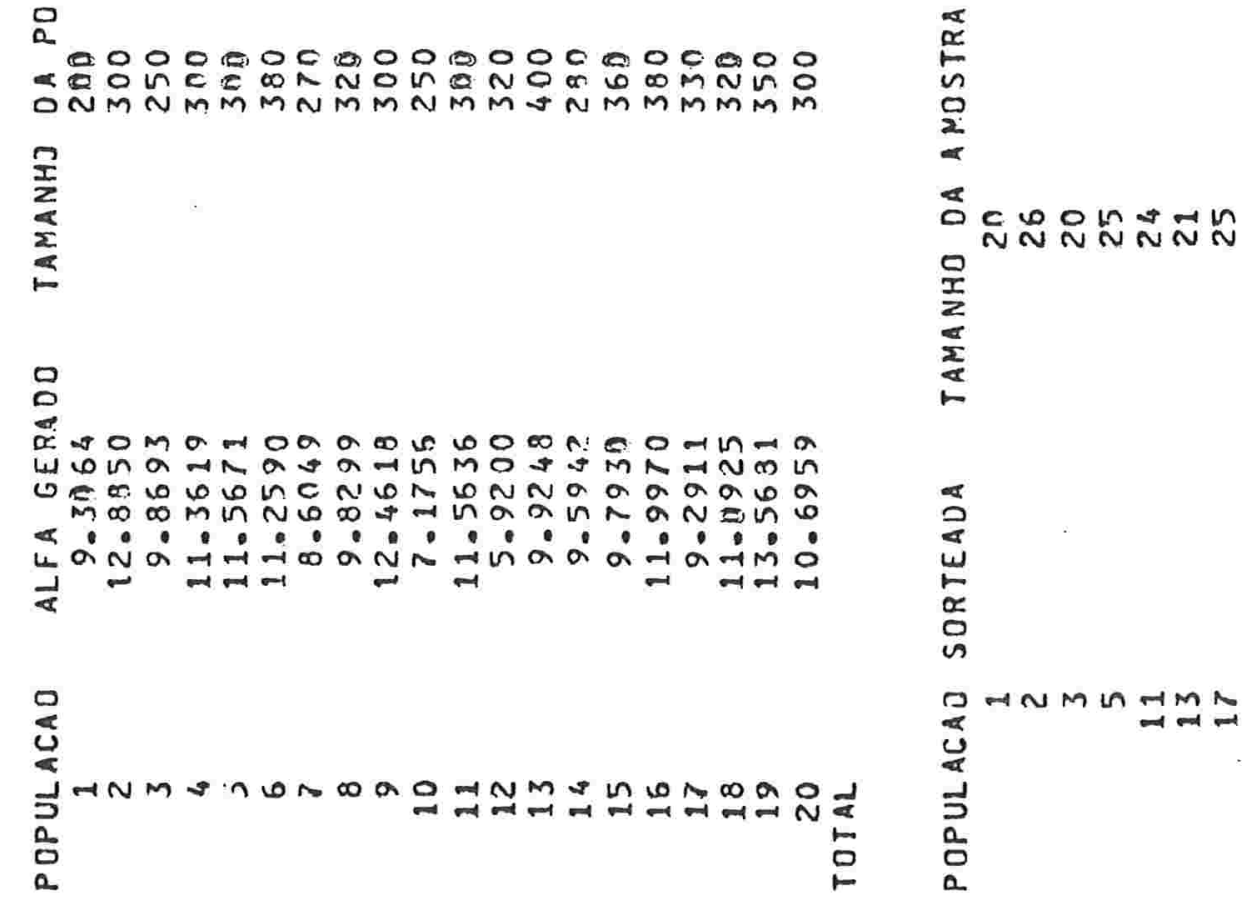
REFERENCIAS BIBLIOGRÁFICAS

ANDRADE, D.F. \& BOLFARINE, H. (1986) Estimation in covariance components models with unequal intraclass variances. Pub. Inst. Statist. Univ. Paris, 31(1):17-26.

BOX, M.J.; DAVIES, D.; SWANN, W.H. eds. (1969) Nonlinear optimization techniques. Edinburgh, Oliver and Boyd. $60 \mathrm{p}$.

CASSEL, C.M.; SARNDAL, C.E.; WRETMAN, J.H. (1977) Foundations of inference in survey sampling. New York, John Wiley. 192p. (Wiley Series in Probability and Mathematical Statistics).

CORDEIRO, G.M. (1986) Modelos lineares generalizados. Campinas, IMECC-UNICAMP. $286 \mathrm{p}$.

DE BRUIJN, N.G. (1958) Asymptotic methods in analysis. Amsterdan, North-Holland. 200p. (Bibliotheca Mathematica, 4).

DE FINETTI, B. (1964) Forsight: its logical laws, its subjective sources. In: KYBURG JR., H.E. \& SMOKLER, H.E. eds. Studies in subjective probability. New York, Wiley. p.93-158.

ELIAN, S.N. (1984) Previsão linear em populações finitas. São Paulo. 172p. Dissertação (Mestrado) - IME-USP. 
IMSL LIBRARY reference manual. (1979) Houston, IMSL.3v.

LEONARD, T. (1972) Bayesian methods for binomial data.

Biometrika, $59(3): 581-89$.

LEONARD, T. (1976) Some alternative approaches to multiparameter estimation. Biometrika, 63 (1):69-75.

LINDLEY, D.V. (1965) Introduction to probability and statistics from a Bayesian viewpoint. Cambridge, University Press, v.2.

LINDLEY, D.V. (1971) The estimation of many parameters. In: GODAMBE, V.P. \& SPROTT, D.A. eds. Foundations of statistical inference. Toronto, Holt. p.435-55.

LINDLEY, D.V. \& SMITH, A.F.M. (1972) Bayes estimates for the linear model: with discussion. J. Royal Statist. Soc. Ser. B, $34(1): 1-41$.

MALEC, D. \& SEDRANSK, J. (1985) Bayesian inference for finite population parameters in multistage cluster sampling. J. Amer. Statist. Assoc., 80 (392):897-902.

MCCULLAGH, P. \& NELDER, J.A. (1983) Generalized linear models. London, Chapman and Hall. 261p. (Monographs on Statistics and Applied Probability) . 
RODRIGUES, J.\& BOLFARINE, H. (1984) Teoria da previsão em populações finitas. Rio de Janeiro, UFRJ. $80 p$.

RODRIGUES, J.; BOLFARINE, H.; ROGATKO, A. (1985) A general theory of prediction in finite populations. Internat. Statist. Rev., $53(3): 239-54$.

SANDOVAL, M.C. (1987) Teoria da previsão em populações com tendência. São Paulo: 63p. Dissertação (Mestrado) - IME-USP.

SCOTT, A. \& SMITH, T.M. (1969) Estimation in multi-stage surveys. J. Amer. Statist. Assoc., 64 (327):830-40.

TIERNEY, L. \& KADANE, J.B. (1986) Accurate approximations for posterior moments and marginal densities. J. Amer. Statist. Assoc., $81(393): 82-6$.

WEST, M. (1985) Generalized linear models: scale parameters, outlier acommodation and prior distributions. In: BERNARDO, J.M. et al. eds. Bayesian statistics: Proceedings of the Second International. Meeting, 2, Valencia, 1983. Amsterdam, North-Holland. p.531-557.

ZELLNER, A. (i971) An introduction to Bayesian inference in econometrics. New York, John Wiley. 431 p. (wiley Series in Probability and Mathematical statistics). 


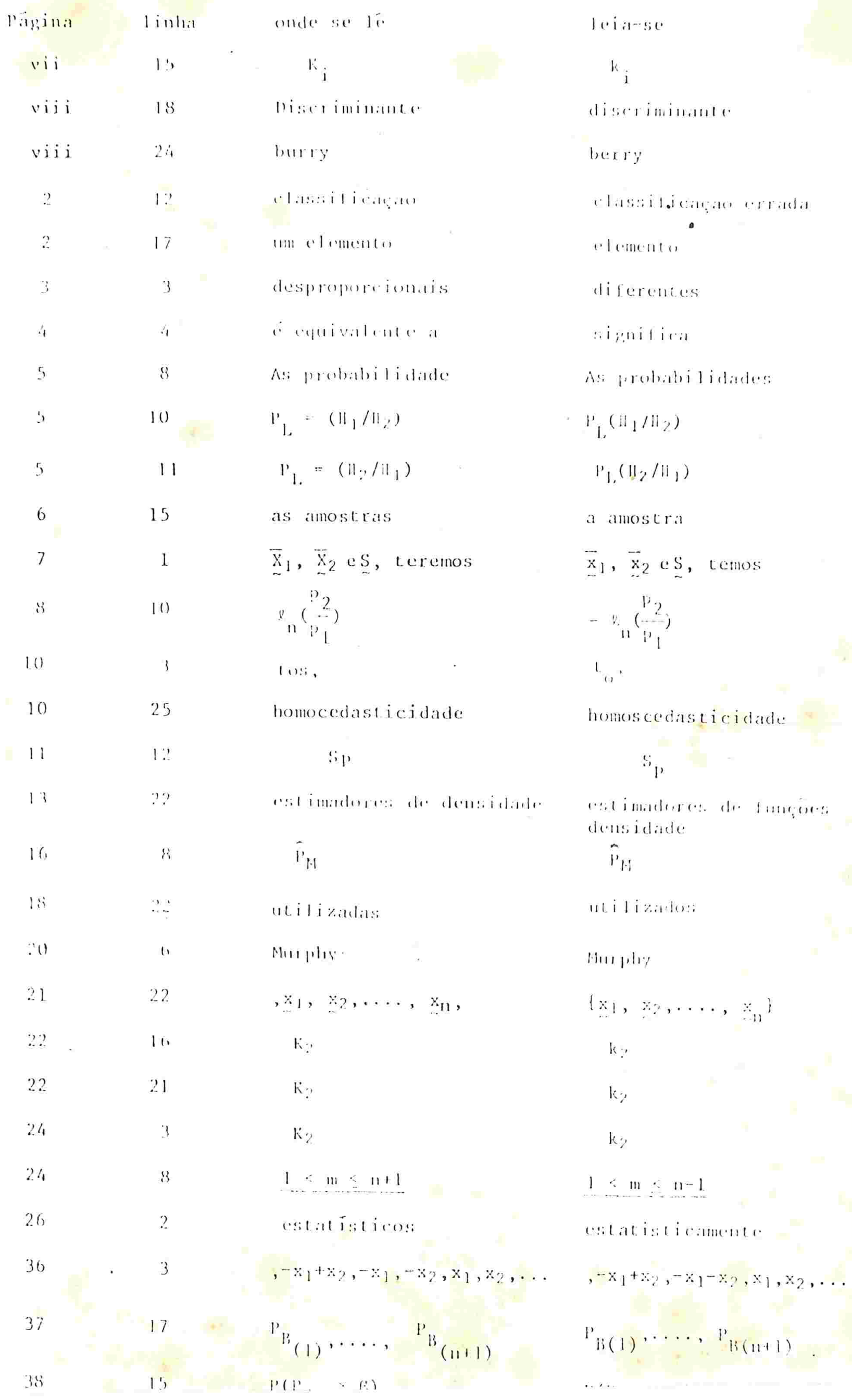


pirina

41

42

42

42

43

44

45

46

46

48

50

52

56

56

57

58

58

59

6)

61.

62

1,1

66

70

71

76

76 linl:1

17

1

3

18

9

1.1

?'

6. 1

15

9)

1

2

21

23

26

20

22

4

13

20

7

(1)

18

19

?

8

17 onde so to

Estimalgio de $(x)$ $S x$

$$
\frac{1}{v_{S X}}
$$

$N_{i}$ observisous

Class if icamos

discrimintmle ख.

$$
\text { 1) - I }
$$

$1_{1}$

Patrick Fischer

do vol lume

$\phi_{8}(:)$

$\phi_{4}(x)=\phi_{8}(x)$

$\operatorname{Sejal} I_{i}(x)$

$\operatorname{Se} f_{i}(x)$

$\hat{f}_{i}(x) \ldots f_{i}(x)$

torna-se

('IIt:1:1

(Fischer)

- métodos sem

Metodo 1

Fixemos

$[(1-19)(11,11)]$

$x_{0}^{1}$

$a, 1,1)$

$1 \cdot\left(1 A_{1}: A\right): y$

$1 \because v$

combinadas leja-ice

listimaçä de $f_{j}(x)$

$s_{x}$<smiles>C[As](C)(C)(C)I</smiles>

$N_{i}$ de observaçoes

classificamos

discriminante dada em

$$
\text { (1) }
$$

1. 9

Patrick e fischer

dos volumes

$$
\begin{aligned}
& =\phi_{8}(x) \\
& \phi_{1}(x)=\phi_{12}(x)
\end{aligned}
$$

Seja $\hat{\mathrm{f}}_{\mathrm{i}}(\mathrm{x})$

$\operatorname{se} \hat{f}_{i}(x)$

$\hat{f}_{1}(x) \ldots f_{1}(x)$

Lornar-se

entre

(Fisher)

- método scim

Método 1

Fixamos

$$
\begin{aligned}
& \because \because \cdots[(1-1, ;)(1 \cdots, 1)] \\
& x^{1} \\
& \left.-a_{1}+1\right) \\
& P(1, \therefore, \quad \therefore) \therefore r \\
& \text { v } \\
& \text { combinadas; }
\end{aligned}
$$




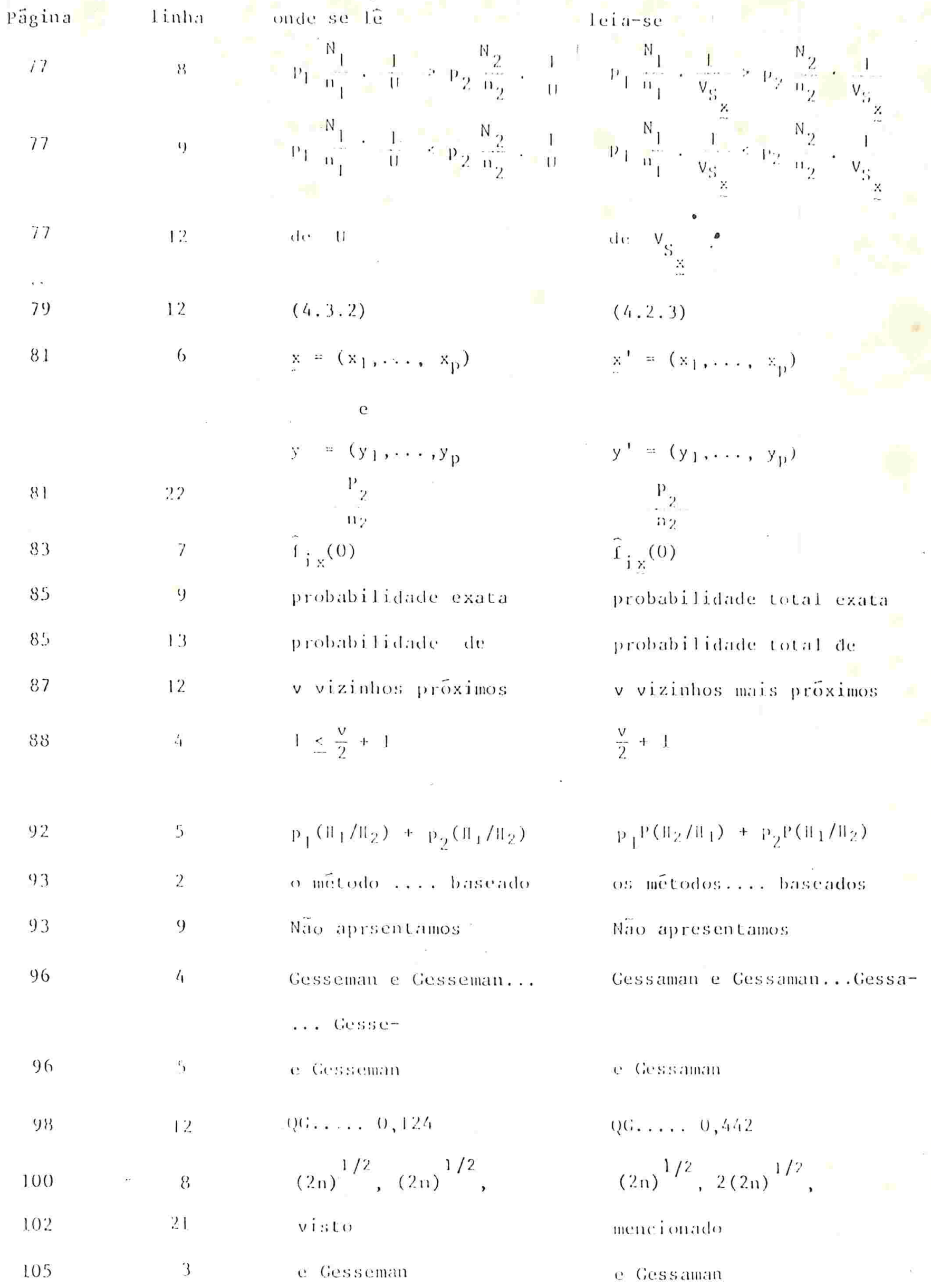


pägina

105

107

107

148

148

149

150

151 linlı)

onde sic in

Método Amostral

parta os métodos de

and a is ados

mensurements

27

Seric

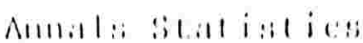

$1 \%$ leiatis

Lincar Amostral.

dos métodos när paramétri co:) de:

analisados e do método quadrätico amostral

measurements

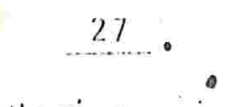

Series

Anlals of Statistics

12

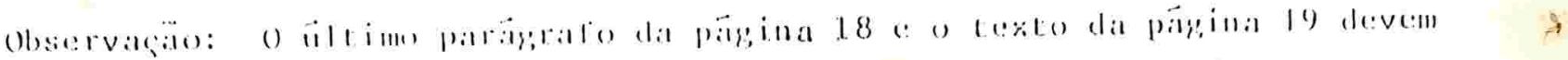
ser lamalefeidos para a pägina 12 e colocados após a linla 22 . 